New Astronomy, 13 (2008), p. $619-638$.

ISSN: $1384-1076$

doi:10.1016/j.newast.2008.03.007

(C) Elsevier. All rights reserved.

http://www.elsevier.com/wps/find/homepage.cws_home

http://www.elsevier.com/locate/newast

\title{
Electron-ion recombination rate coefficients and photoionization cross-sections for Al XI, Al XII, Si XII, Si XIII for UV and X-ray modeling
}

\author{
Sultana N. Nahar * \\ Department of Astronomy, The Ohio State University, Columbus, OH 43210, United States
}

\begin{abstract}
Results are presented from detailed study of inverse processes of photoionization and electron-ion recombination of $(h v+\mathrm{Al} \mathrm{XI} \rightleftharpoons \mathrm{Al} \mathrm{XII}+\mathrm{e}),(h v+\mathrm{Al} \mathrm{XII} \rightleftharpoons \mathrm{Al} \mathrm{XIII} \mathrm{+} \mathrm{e}),(h v+\mathrm{Si} \mathrm{XII} \rightleftharpoons \mathrm{Si} \mathrm{XIII} \mathrm{+} \mathrm{e})$, and $(h v+\mathrm{Si} \mathrm{XIII} \rightleftharpoons \mathrm{Si} \mathrm{XIV}+\mathrm{e})$ using ab initio unified method. These are the first results on photoionization cross-sections $\left(\mathrm{O}_{\mathrm{PI}}(\mathrm{n} S L J)\right)$ with autoionizing resonances and level-specific recombination rate coefficients, incorporating both the radiative and dielectronic recombination, for these ions. All fine structure levels with n 610 and $06 l 69$ are considered. A total of 98 fine structure levels with $1 / 2 \leq J \leq 17 / 2$ for Al XI and Si XII, and 190 for Al XII and 189 for Si XIII with $0 \leq J \leq 10$ are found. $\mathrm{O}_{\mathrm{PI}}(n S L J)$ show background enhancements due to core excitations and narrow high-peak resonances. Level-specific recombination rates show smooth decay with a small bump at high temperature. Present total recombination rate coefficients with temperature $\left(\alpha_{R}(T)\right)$ show good agreement with available rates. Recombination rates over photoelectron energy $\left(\alpha_{R}(E)\right)$ are presented for astrophysical and laboratory plasma applications. Total recombination rates for H-like Al XIII and Si XIV are given for completeness. Calculations are carried out in the relativistic Breit-Pauli $R$-matrix method using coupled channel wavefunctions. Inclusion of important atomic effects such as radiation damping, channel couplings, interference of DR and RR, and relativistic fine structure effects should provide accuracy within $10-15 \%$. The comprehensive data-sets are applicable for various models such as for ionization balance and recombination-cascade for UV and X-ray lines.
\end{abstract}

(C) 2008 Elsevier B.V. All rights reserved.

\section{Introduction}

Highly charged He-like and Li-like ions, especially from carbon to iron, are most prevalent in high temperature astrophysical and laboratory sources. The X-ray spectra taken by space observatories Chandra, XMM-Newton contain many diagnostics lines of these ions (Lee et al., 2001; Ness et al., 2003; Hillier, 2005). For example, the well-known $K \alpha$ spectral lines in X-rays involve transitions from the $\mathrm{n}=2$ levels in He-like ions and provides information of temperature, density, and ionization state of the plasma (e.g. Oelgoetz and Pradhan, 2001, 2004). The ionization balance in the source requires knowledge of ionization and electron-ion recombination rates for the ionization stage of He-like as well as Li-like and H-like stages. Emission lines in general contain a component due to electron-ion recombination, particularly in transient plasmas out of ionization equilibrium. To model the recombination component, recombination-cascade coefficients are computed from level-specific recombination rate coefficients for a large number of excited levels, as presented in this report.

Present work reports detailed study of the inverse processes of photoionization and electron-ion recombination using the unified method (Nahar and Pradhan, 1994) which enables 
self-consistent set of atomic data for both the processes. Compared to available separate rates for radiative recombination ( $R R$ ) and dielectronic recombination (DR), unified method provides the total rates in an ab initio and unified manner. The aim of the present work is to study the highly charged astrophysically abundant He-and Li-like aluminium and silicon ions and provide accurate and self-consistent results. Relativistic effects and radiation damping are important for these ions. The unified method is implemented through the powerful relativistic Breit-Pauli $R$-matrix (BPRM) method. Earlier calculations for photoionization cross-sections for these ions were carried out under the Opacity Project by Fernley et al. (1987) for He-like ions and by Peach et al. (1988) for Li-like ions. They were computed in the non-rel-ativistic $L S$ coupling approximation and using wavefunctions smaller than the present ones. Existing recombination rates available are for total radiative recombination from central field approximation (e.g. Verner and Ferland, 1996), and for total dielectronic recombination using isolated resonance approximation (e.g. Romanik, 1988).

The present results should be useful for spectral analysis of X-ray observations from Chandra X-ray Observatory and XMM-Newton, and UV observations, such as from the Far Ultraviolet Spectroscopic Explorer (FUSE), the Solar and Heliospheric Observatory (SOHO), etc. The data are presented at all energies and temperatures prevalent in high temperature sources such as Active Galactic Nuclei, supernova remnants, hot stellar coronae, etc. (e.g. Canizares et al., 2000).

\section{Theory}

The inverse processes of photoionization and electron-ion recombination can proceed in two ways: directly as (i),

$$
\mathrm{e}+X^{++} \leftrightharpoons h v+X^{+}
$$

where right arrow indicates the direct or radiative recombination (RR) and the left arrow indicates photoionization, or (ii) through an intermediate doubly excited autoionizing state as

$$
\mathrm{e}+X^{++} \leftrightharpoons\left(X^{+}\right)^{* *} \leftrightharpoons \begin{cases}(\mathrm{a}) & \mathrm{e}+X^{++}(\mathrm{AI}) \\ \text { (b) } & h v+X^{+}(\mathrm{DR})\end{cases}
$$

The autoionizing state leads either to autoionization (AI) where the electron goes free or to dielectronic recombination (DR) by emission of a photon. The state introduces a resonance in photoionization and recombination cross-sections. RR and DR are inseparable in nature. The unified method subsumes both the RR and DR processes and considers photoionization from and recombination into the infinity of ( $\mathrm{e}+\mathrm{ion})$ levels. It is an ab initio method implemented in the close coupling (CC) approximation and R-matrix method (Nahar and Pradhan, 1992, 1994) as briefly outlined below.

In the CC approximation, the atomic system of the ion and an interacting electron, is described by an $\mathrm{N}$-electron system called the target (core) interacting with the $(\mathrm{N}+1)$ th electron which can be bound to the ion or in the continuum. The total wavefunc-tion, $\Psi(E)$, of the $(\mathrm{N}+1)$ electron-ion system of symmetry $J \pi$ is represented by an expansion of target eigenfunctions, $\chi_{\mathrm{i}}$, as

$$
\Psi(\text { ion }+\mathrm{e} ; E)=A \sum_{i} \chi_{i}(\text { ion }) \theta_{i}+\sum_{j} c_{j} \Phi_{j}(\text { ion }+\mathrm{e})
$$


where the target is in a specific level $J_{i} \pi_{i}$. is the wavefunction of the $(\mathrm{N}+1)$ th electron in a channel, $\operatorname{SiL}_{i}(J i) \pi i k_{i}^{2} \ell i(J \pi)$ and $k_{i}^{2}$ is its kinetic energy. The channel is called open or closed depending on positive or negative energy $\left(k_{i}^{2}\right)$. The autoionizing resonances result from the couplings of open and closed channels. The $\Phi_{\mathrm{j}}$ 's are correlation functions of the $(\mathrm{N}+1)$-electron system and account for short range correlations and orthogonality between continuum and bound orbitals.

The relativistic Hamiltonian, in the Breit-Pauli R-matrix (BPRM) method as adopted under the Iron Project (Hummer et al., 1993), is given by

$$
H_{N+1}^{\mathrm{BP}}=H_{N+1}^{\mathrm{NR}}+H_{N+1}^{\mathrm{mass}}+H_{N+1}^{\mathrm{Dar}}+H_{N+1}^{\mathrm{so}} .
$$

where first term, $H_{N+1}^{N R}$, , is the nonrelativistic Hamiltonian,

$$
H_{N+1}^{N R}=\sum_{i=1}^{N+1}\left\{-\nabla_{i}^{2}-\frac{2 Z}{r_{i}}+\sum_{j>i}^{N+1} \frac{2}{r_{i j}}\right\}
$$

and the additional relativistic one-body correction terms are the mass correction, $H^{\text {mass }}=-\frac{\alpha^{2}}{4} \sum_{i} p_{i}^{4}, \quad$, Darwin, $H^{\mathrm{Dar}}=\frac{7 \alpha^{2}}{4} \sum_{i} \nabla^{2}\left(\frac{1}{r_{i}}\right)$ and spin-orbit interaction, $H^{\mathrm{so}}=Z \alpha^{2} \sum_{i} \frac{1}{r^{3}} \mathbf{l}_{i} \cdot \mathbf{s}_{i} \cdot$. The spin-orbit term splits the LS term in to fine structure components. The set of $S L \pi$ is recoupled for $J \pi$ levels of (e + ion)-system which is followed by diagonalization of the Hamiltonian, $H_{N+1}^{\mathrm{EP}} \Psi=E \Psi$. . The positive energy $\left(\mathrm{E}=k^{2} \geq 0\right)$ and negative energy $(\mathrm{E}<0)$ define the continuum $\Psi_{F}$ or bound $\Psi_{B}$ states which are used in the transition matrix elements.

The reduced matrix element for the bound-free transition is obtained as $\left\langle\Psi_{\mathrm{B}}\|\mathbf{D}\| \Psi_{\mathrm{F}}\right\rangle$ where $\mathbf{D}$ $=\sum_{i} \mathrm{r}_{\mathrm{i}}$ is the dipole operator, the sum is the number of electrons. The dipole line strength, $\mathbf{S}=\left|\left\langle\Psi_{\mathrm{B}}\|\mathbf{D}\| \Psi_{\mathrm{F}}\right\rangle\right|^{2}$, , gives the photoionization cross-section, $\sigma_{\mathrm{Pl}}=\left[4 \pi^{2} \mid\left(3 \mathrm{~g}_{\mathrm{i}} \mathrm{c}\right)\right] \omega \mathbf{S}$, 1 , where gi is the statistical weight factor of the initial bound state.

Radiation damping of the resonances, which is important for the present $\mathrm{H}$ - and the He-like recombining ions, is considered using a resonance fitting procedure (Sakimoto et al., 1990; Pradhan and Zhang, 1997; Zhang et al., 1999). The radiative decay rates $\left(A_{r}\right)$ for transitions, $2 \mathrm{p}\left({ }^{2} \mathrm{P}\right.$ ${ }_{3 / 2,1 / 2}^{\circ} \rightarrow 1 \mathrm{~s}\left({ }^{2} \mathrm{~S}_{1 / 2}\right)$ in $\mathrm{H}$-like core and $1 \mathrm{~s} 2 \mathrm{p}\left({ }^{1} \mathrm{P}_{1}^{0}\right) \rightarrow 1 \mathrm{~s}^{2}\left({ }^{1} \mathrm{~S}_{1 / 2}\right)$ in He-like core are comparable to autoioniza-tion rates, typically $A_{a} \sim 10^{12}-10^{14} \mathrm{~s}^{-1}$, causing autoionizing resonances to damp radiatively to a significant extent. All resonances, up to effective quantum number $\mathrm{v} \leq 10$, have been damped. Resonances beyond $\mathrm{v}>10$ are treated with DR theory discussed below. 
Table 1

Target levels in the eigenfunction expansions of Al XI, Si XII, Al XII and Si XIII and their energies in Ry, calculated $\mathrm{E}_{\mathrm{c}}$ and observed $\mathrm{E}_{\mathrm{o}}$ (NIST compilation)

\begin{tabular}{|c|c|c|c|c|c|c|c|c|c|c|}
\hline & \multirow[t]{2}{*}{ Level } & \multicolumn{2}{|l|}{ Al XII } & \multicolumn{2}{|l|}{ Si XIII } & \multirow[t]{2}{*}{ level } & \multicolumn{2}{|l|}{$\mathrm{Al} \mathrm{XII}$} & \multicolumn{2}{|l|}{ Si XIV } \\
\hline & & $E_{0}$ & $E_{c}$ & $E_{\mathrm{o}}$ & $E_{c}$ & & $E_{\mathrm{o}}$ & $E_{c}$ & $E_{0}$ & $E_{c}$ \\
\hline 1 & $1 \mathrm{~s}^{21} \mathrm{~S}_{0}$ & 0.0 & 0.0 & 0.0 & 0.0 & $1 s^{2} S_{1 / 2}$ & 0.0 & 0.0 & 0.0 & 0.0 \\
\hline 2 & $1 s 2 s^{3} S_{1}$ & 115.75881 & 115.82 & 135.19508 & 135.29 & $2 \mathrm{p}^{2} \mathrm{P}_{1 / 2}^{0}$ & 126.983 & 127.01 & 147.315 & 147.35 \\
\hline 3 & $1 \mathrm{~s} 2 \mathrm{p}^{3} \mathrm{P}_{0}^{\mathrm{o}}$ & 116.71369 & 116.75 & 136.2322 & 136.30 & $2 s^{2} S_{1 / 2}$ & 126.986 & 127.01 & 147.320 & 147.35 \\
\hline 4 & $1 \mathrm{~s} 2 \mathrm{p}^{3} \mathrm{P}_{1}^{0}$ & 116.72500 & 116.76 & 136.24851 & 136.31 & $2 \mathrm{p}^{2} \mathrm{P}_{3 / 2}^{0}$ & 127.078 & 127.11 & 147.444 & 147.48 \\
\hline 5 & $1 \mathrm{~s} 2 \mathrm{p}^{3} \mathrm{P}_{2}^{\mathrm{o}}$ & 116.77170 & 116.81 & 136.31363 & 136.38 & $3 \mathrm{p}^{2} \mathrm{P}_{1 / 2}^{0}$ & 150.531 & 150.56 & 174.640 & 174.68 \\
\hline 6 & $1 \mathrm{~s} 2 \mathrm{~s}^{1} \mathrm{~S}_{0}$ & 116.7858 & 116.81 & 136.31419 & 136.36 & $3 s^{2} s_{1 / 2}$ & 150.532 & 150.56 & 174.642 & 174.68 \\
\hline 7 & $1 \mathrm{~s} 2 \mathrm{p}^{1} \mathrm{P}_{1}^{0}$ & 117.47218 & 117.54 & 137.0731 & 137.17 & $3 d^{2} D_{3 / 2}$ & 150.560 & 150.59 & 174.679 & 174.72 \\
\hline 8 & $1 \mathrm{~s} 3 \mathrm{~s}^{3} \mathrm{~S}_{1}$ & 136.87653 & 136.93 & 159.89761 & 159.98 & $3 \mathrm{p}^{2} \mathrm{P}_{3 / 2}^{0}$ & 150.560 & 150.59 & 174.679 & 174.75 \\
\hline 9 & $1 \mathrm{~s} 3 \mathrm{~s}^{1} \mathrm{~S}_{0}$ & 137.14804 & 137.19 & 160.19315 & 160.26 & $3 d^{2} D_{5 / 2}$ & 150.569 & 150.60 & 174.692 & 174.73 \\
\hline 10 & $1 s 3 p^{3} P_{2}^{0}$ & 137.15619 & 137.21 & 160.20703 & 160.29 & $4 p^{2} P_{1 / 2}^{o}$ & 158.770 & 158.80 & 184.199 & 184.24 \\
\hline 11 & $1 s 3 p^{3} P_{1}^{0}$ & 137.14236 & 137.19 & 160.18770 & 160.27 & $4 s^{2} s_{1 / 2}$ & 158.770 & 158.80 & 184.200 & 184.24 \\
\hline 12 & $1 \mathrm{~s} 3 \mathrm{p}^{3} \mathrm{P}_{0}^{0}$ & 137.13881 & 137.19 & 160.18279 & 160.27 & $4 d^{2} D_{3 / 2}$ & 158.781 & 158.81 & 184.215 & 184.25 \\
\hline 13 & $1 \mathrm{~s} 3 \mathrm{~d}^{3} \mathrm{D}_{1}$ & 137.30050 & 137.19 & 160.36480 & 160.45 & $4 \mathrm{p}^{2} \mathrm{P}_{3 / 2}^{0}$ & 158.781 & 158.81 & 184.215 & 184.25 \\
\hline 14 & $1 \mathrm{~s} 3 \mathrm{~d}^{3} \mathrm{D}_{2}$ & 137.30092 & 137.35 & 160.36506 & 160.45 & $4 \mathrm{f}^{2} \mathrm{~F}_{5 / 2}^{\mathrm{o}}$ & 158.785 & 158.81 & 184.221 & 184.26 \\
\hline 15 & $1 \mathrm{~s} 3 \mathrm{~d}^{3} \mathrm{D}_{3}$ & 137.30604 & 137.36 & 160.37252 & 160.46 & $4 d^{2} D_{5 / 2}$ & 158.785 & 158.81 & 184.221 & 184.26 \\
\hline 16 & $1 \mathrm{~s} 3 \mathrm{~d}^{1} \mathrm{D}_{2}$ & 137.31310 & 137.36 & 160.37941 & 160.47 & $4 \mathrm{r}^{2} \mathrm{~F}_{7 / 2}^{0}$ & 158.787 & 158.82 & 184.223 & 184.26 \\
\hline 17 & $1 \mathrm{~s} 3 \mathrm{p}^{1} \mathrm{P}_{1}^{\mathrm{o}}$ & 137.34746 & 137.41 & 160.41419 & 160.51 & & & & & \\
\hline
\end{tabular}

The correlation configurations and values of the $\lambda$ parameters of the orbitals are given below.

Al XII: correlations $-2 \mathrm{~s}^{2}, 2 \mathrm{p}^{2}, 3 \mathrm{~s}^{2}, 3 \mathrm{p}^{2}, 3 \mathrm{~d}^{2}, 2 \mathrm{~s} 2 \mathrm{p}, 2 \mathrm{~s} 3 \mathrm{~s}, 2 \mathrm{~s} 3 \mathrm{p}, 2 \mathrm{~s} 3 \mathrm{~d}, 2 \mathrm{~s} 4 \mathrm{~s}, 2 \mathrm{~s} 4 \mathrm{p}, 2 \mathrm{p} 3 \mathrm{~s}, 2 \mathrm{p} 3 \mathrm{p}, 2 \mathrm{p} 3 \mathrm{~d}, 2 \mathrm{p} 4 \mathrm{~s}, 2 \mathrm{p} 4 \mathrm{p}$. $\lambda_{\mathrm{nl}}-0.991(1 \mathrm{~s}), 0.991(2 \mathrm{~s}), 1(2 \mathrm{p}), 1(3 \mathrm{~s}), 1(3 \mathrm{p}), 1(3 \mathrm{~d}), 1(4 \mathrm{~s}), 1(4 \mathrm{p})$.

Si XIII: correlations $-2 \mathrm{~s}^{2}, 2 \mathrm{p}^{2}, 3 \mathrm{~s}^{2}, 3 \mathrm{p}^{2}, 3 \mathrm{~d}^{2}, 2 \mathrm{~s} 2 \mathrm{p}, 2 \mathrm{~s} 3 \mathrm{~s}, 2 \mathrm{~s} 3 \mathrm{p}, 2 \mathrm{~s} 3 \mathrm{~d}, 2 \mathrm{~s} 4 \mathrm{~s}, 2 \mathrm{~s} 4 \mathrm{p}, 2 \mathrm{p} 3 \mathrm{~s}, 2 \mathrm{p} 3 \mathrm{p}, 2 \mathrm{p} 3 \mathrm{~d}, 2 \mathrm{p} 4 \mathrm{~s}, 2 \mathrm{p} 4 \mathrm{p}$.

$\lambda_{\mathrm{nl}}-0.991(1 \mathrm{~s}), 0.991(2 \mathrm{~s}), 1(2 \mathrm{p}), 1(3 \mathrm{~s}), 1(3 \mathrm{p}), 1(3 \mathrm{~d}), 1(4 \mathrm{~s}), 1(4 \mathrm{p})$.

The electron-ion recombination cross-section $\sigma_{\mathrm{RC}}$ can be obtained from photoionization cross-sections $\sigma_{\mathrm{PI}}$ using principle of detailed balance as, in Rydberg units,

$$
\sigma_{\mathrm{RC}}(\epsilon)=\frac{\alpha^{2}}{4} \frac{g_{\mathrm{i}}}{g_{\mathrm{j}}} \frac{(\epsilon+I)^{2}}{\epsilon} \sigma_{\mathrm{Pl}}
$$

Table 2

Radiative decay rates, $A_{\mathrm{fi}}\left(\right.$ in s $^{-1}$ ) for allowed core transitions to the ground level, $1 \mathrm{~s}^{21} \mathrm{~S}_{0}$ for Al XII, Si XIII, and $1 \mathrm{~s}^{2} \mathrm{~S} 1 / 2$ for Al XIII, Si XIV

\begin{tabular}{|c|c|c|}
\hline \multirow[t]{2}{*}{ Level } & \multicolumn{2}{|l|}{$\underline{A_{f i}}$} \\
\hline & Al XII & Si XIII \\
\hline $\left.1 \mathrm{~s} 2 \mathrm{p}^{(3} \mathrm{P}_{1}^{\mathrm{o}}\right)$ & $6.60(10)$ & $1.39(11)$ \\
\hline $1 \mathrm{~s} 2 \mathrm{p}\left({ }^{1} \mathrm{P}_{1}^{\mathrm{o}}\right)$ & $2.76(13)$ & $3.78(13)$ \\
\hline $1 \mathrm{~s} 3 \mathrm{p}\left({ }^{3} \mathrm{P}_{1}^{0}\right)$ & $9.18(10)$ & $4.20(10)$ \\
\hline \multirow[t]{2}{*}{$1 \mathrm{~s} 3 \mathrm{p}\left({ }^{1} \mathrm{P}_{1}^{\mathrm{o}}\right)$} & $7.90(12)$ & $1.08(13)$ \\
\hline & Al XIII & Si XIV \\
\hline $2 \mathrm{p}\left({ }^{2} \mathrm{P}_{1 / 2}^{0}\right)$ & $1.78(13)$ & $2.40(13)$ \\
\hline $\left.2 \mathrm{p}^{2} \mathrm{P}_{32}^{0}\right)$ & $1.79(13)$ & $2.40(13)$ \\
\hline $\left.3 p^{\prime 2} P_{12}^{0}\right)$ & $3.68(12)$ & $6.28(12)$ \\
\hline $\left.3 p^{(2} P_{3,2}^{0}\right)$ & $4.72(12)$ & $6.34(12)$ \\
\hline $\left.4 p^{\prime 2} P_{12}^{0}\right)$ & $1.85(12)$ & $2.46(12)$ \\
\hline $4 \mathrm{p}^{2}\left(\mathrm{P}_{32}^{\mathrm{o}}\right)$ & $1.88(12)$ & $2.51(12)$ \\
\hline
\end{tabular}


which is known as the Milne relation. Here $\alpha$ is the fine structure constant, $\epsilon$ is the photoelectron energy, $g j$ is the statistical weight factor of the recombined ion and $I$ is the ionization potential. $\sigma_{\mathrm{RC}}$ computed from $\sigma_{\mathrm{PI}}$ with sufficiently large number of energies to delineate the non-resonant background and the autoionizing resonances represents both RR and DR processes. For the recombining ion in its ground state, $\sigma_{\mathrm{P}} \mathrm{I}$ corresponds to partial photoionization cross-sections, leaving the ion in the ground state. Recombination rate coefficients, $\alpha_{R}(n S L J)$, of individual recombined levels are obtained by convolving $\sigma_{\mathrm{RC}}$ over Maxwellian electron distribution

$$
\begin{aligned}
& f(v)=\frac{4}{\sqrt{\pi}}\left(\frac{m}{2 k T}\right)^{3 / 2} v^{2} \mathrm{e}^{\frac{-m v^{2}}{2 k T}} \text { at a given temperature as } \\
& \alpha_{\mathrm{R}}(i, T)=\int_{0}^{\infty} v f(v) \sigma_{R C}(i) \mathrm{d} v,
\end{aligned}
$$

where $i=n S L J$. The sum of these individual rates $\sum_{\mathrm{i}} \alpha_{\mathrm{R}}(i, T)$ provides the contribution to the total recombination rate coefficient, $\alpha_{\mathrm{R}}$ and the total recombination cross-sections, $\sigma_{\mathrm{RC}}$.

Total recombination rates of the electron-ion system correspond to contributions from infinite number of recombined states. In unified method, the recombined states are divided into two groups: group (A) states $\mathrm{n} \leq \mathrm{n}_{\mathrm{o}}$ with all possible fine structure $J \pi$ symmetries and group (B) states with $\mathrm{n}_{\mathrm{o}}<\mathrm{n}<\infty$, with $\mathrm{n}_{\mathrm{o}} \sim 10$. 


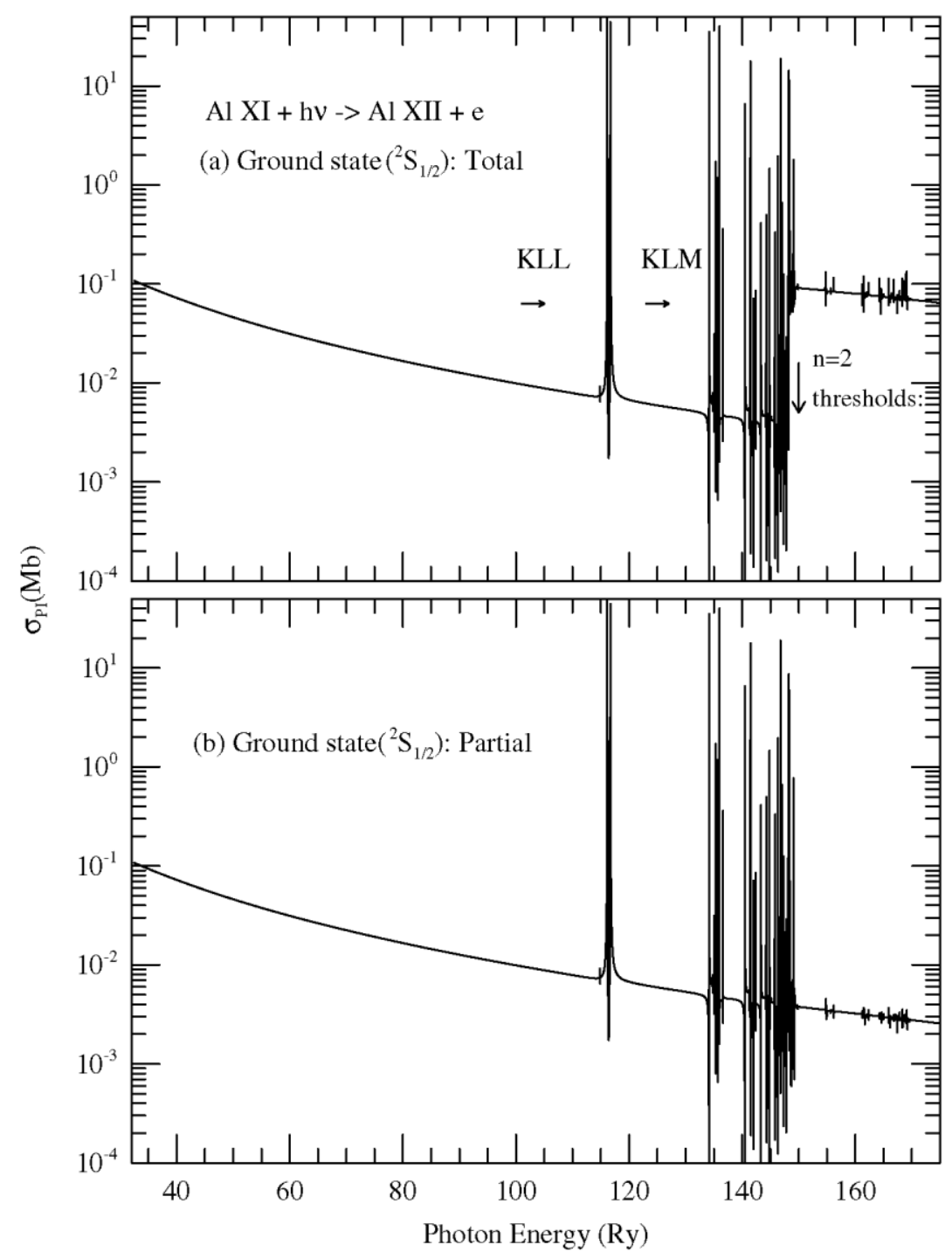

Fig. 1. (a) Total and (b) partial photoionization cross-sections of the ground level $1 \mathrm{~s}^{2} 2 \mathrm{~s}\left({ }^{2} \mathrm{~S}_{1 / 2}\right)$ of $\mathrm{Al} \mathrm{XI}$. The large enhancement in the total $r_{P I}$ around $\mathrm{n}=2$ thresholds $(\sim 175.5 \mathrm{Ry})$ is due to K-shell ionization $\left(h v+1 s^{2} 2 l \rightarrow 1 \mathrm{~s} 2 l\right.$ $+\mathrm{e})$. No jump is in partial cross-section since ionization in to the excited core are not included.

The recombination rates coefficients of group (A) states are obtained from the photoionization cross-sections as described above.

Group (B) levels, $\mathrm{n}_{\mathrm{o}}<\mathrm{n}<\infty$, are treated through quantum defect theory of DR within the close coupling approximation (Bell and Seaton, 1985; Nahar and Pradhan, 1992,1994). The energy region is dominated by narrow high- $n$ resonances due to DR. The background contribution of these levels is negligible except at very low temperature. The DR contributions from these levels are obtained from the collision strengths, $\Omega(\mathrm{DR})$, using radiation damping theory as

$$
\Omega(\mathrm{DR})=\sum_{S L \pi} \sum_{n}(1 / 2)(2 S+1)(2 L+1) \mathrm{P}_{n}^{S L \pi}(\mathrm{DR})
$$


where the DR probability $\mathrm{P}_{n}^{\mathrm{SL} \pi}$ in entrance channel $\mathrm{n}$ is, $P_{n}^{\mathrm{LL} \pi}(\mathrm{DR})=\left(1-\mathscr{S}_{\mathrm{ee}}^{\dagger} \mathscr{S}_{\mathrm{ee}}\right)_{n}, \mathscr{S}_{\mathrm{ee}}$ is the matrix for electron scattering including radiation damping. The cross-section, $\sigma_{\mathrm{RC}}$ in $\mathrm{Mb}$, and collision strength, $\Omega_{R C}$, for recombination are related as

$$
\sigma_{\mathrm{RC}}(i \rightarrow j)(\mathrm{Mb})=\frac{\pi}{g_{i} k_{i}^{2}} \Omega_{\mathrm{RC}}(i, j)\left(a_{0}^{2} / 10^{-18}\right),
$$

where $k_{i}^{2}$ is in Rydbergs. For numerical stability, $\Omega_{\mathrm{RC}}$ is preferable to $\sigma_{\mathrm{RC}}$ for calculation of recombination rate coefficient since $\sigma_{R C}$ diverges at zero-photoelectron energy. Background photoionization cross-sections for the high-n group (B) levels are computed hydro-genically, and the corresponding contribution to $\alpha_{R}$ is referred to as the "high-n top-up" (Nahar, 1996).

The total recombination cross-sections $\sigma_{\mathrm{RC}}$, summed from contributions of group A and B states, is an important quantity as it provides information on satellite lines (Nahar and Pradhan, 2006) and the recombination rates $\sigma_{\mathrm{RC}}(\mathrm{E})$ which can be measured experimentally in terms of the photoelectron energy $(\mathrm{E})$. The velocity $(v)$ or energy dependent photorecombination rate can be obtained as

$$
\alpha_{\mathrm{RC}}(E)=v \sigma_{\mathrm{RC}}(E)
$$

The measurable feature is the resonant part of $\alpha_{\mathrm{RC}}(\mathrm{E})$ convolved with the monochromatic bandwidth of the experimental beam in synchrotron facilities such as the Test Storage Ring in Heidelberg (e.g. Pradhan et al., 2001).

\section{Computation}

The calculations for photoionization and electron-ion recombination involve number of stages. The first step is to obtain the target, or the core, orbital wavefunctions. These wavefunctions are 


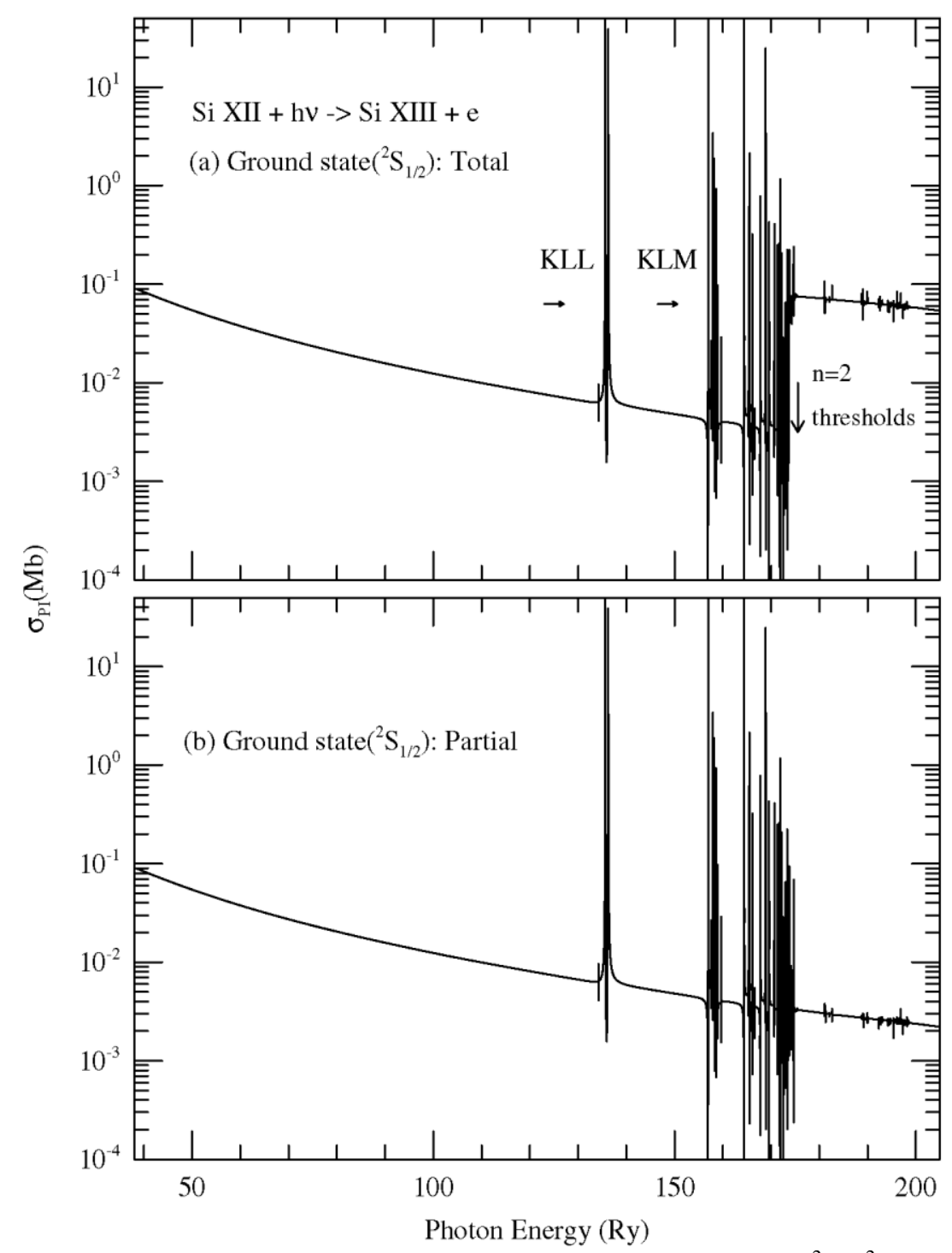

Fig. 2. (a) Total and (b) partial photoionization cross-sections of the ground level $1 \mathrm{~s}^{2} 2 \mathrm{~s}\left({ }^{2} \mathrm{~S}_{1 / 2}\right)$ of Si XII. The large enhancement in the total $\sigma_{P I}$ around $\mathrm{n}=2$ thresholds $(\sim 175.5 \mathrm{Ry})$ is due to K-shell ionization $\left(h v+1 \mathrm{~s}^{2} 2 l \rightarrow 1 \mathrm{~s} 2 l+\mathrm{e}\right)$. No jump is in partial cross-section since ionization in to the excited core are not included.

obtained from configuration interaction atomic structure calculations using the code SUPERSTRUCTURE (Eissner et al., 1974).

The wavefunction expansions for the Li-like Al XI and Si XII contain 17 fine structure levels of the spectroscopic configurations $1 \mathrm{~s}^{2}, 1 \mathrm{~s} 2 \mathrm{~s}, 1 \mathrm{~s} 2 \mathrm{p}, 1 \mathrm{~s} 3 \mathrm{~s}, 1 \mathrm{~s} 3 \mathrm{p}$, and $1 \mathrm{~s} 3 \mathrm{~d}$ of the target core Al XII and Si XIII, respectively. The levels and their energies are given in Table 1 . The set of correlation configurations and the Thomas-Fermi scaling parameters for each orbital in the atomic structure calculations are also given in Table 1 . As seen in Table 1, the calculated energies are very close, within $0.1 \%$, to the observed values compiled in the NIST Compiled Database website: http://www.nist.gov. However, the observed energies used as they provide more accurate resonance positions.

The second term of the wavefunction in Eq. (3) representing bound state correlation functions includes all possible $(\mathrm{N}+1)$-particle configurations with 0 to maximum orbital 
occupancies of $2 \mathrm{~s}^{2}, 2 \mathrm{p}^{2}, 3 \mathrm{~s}^{2}, 3 \mathrm{p}^{2}, 3 \mathrm{~d}^{2}, 4 \mathrm{~s}$ and $4 \mathrm{p}$ for both Al XI and Si XII. The radial integrals for the partial wave expansion are specified for $0 \leq l \leq 9$, with a $R$-matrix basis set of 40 continuum functions. The R-matrix boundary was chosen to be large enough, to $5 a_{\mathrm{o}}$ for cores $\mathrm{Al} \mathrm{XII}$ and $\mathrm{Si}$ XIII, to accomodate the bound orbitals. Computations are carried out for all angular momenta, $0 \leq$ $L \leq 11,1 / 2 \leq J \leq 17 / 2$.

The coupled channel wavefunction expansions for Al XII and Si XIII contain 16 fine structure levels of Al XIII and Si XIX of configurations 1s, 2s, 2p, 3s, 3p, 3d, 4s, 4p, 4d and 4f. The levels and their energies are given in Table 1. For single electron hydrogen-like systems, such as the core Al XIII and Si XIV, accurate energies are obtained easily and hence correlation configurations are not necessary. The Thomas-Fermi parameter for the orbitals were taken to be unity. The bound state correlation functions included all possible configurations from 0 to maximum orbital occupancies of $1 \mathrm{~s}^{2}, 2 \mathrm{~s}^{2}, 2 \mathrm{p}^{2}, 3 \mathrm{~s}^{2}, 3 \mathrm{p}^{2}, 3 \mathrm{~d}^{2}, 4 \mathrm{~s}^{2}, 4 \mathrm{p}^{2}, 4 \mathrm{~d}$ and $4 \mathrm{f}$. The partial wave expansion includes all orbitals $0 \leq l \leq 9$, with a $R$-matrix basis set of 30 continuum functions. The R-matrix boundary was chosen to be $7 \mathrm{a}_{\mathrm{o}}$ for core $\mathrm{Al}$ XIII and $6 \mathrm{a}_{\mathrm{o}}$ for core Si XIV. Computations are carried out for all angular momenta, $0 \leq L \leq 14,0 \leq J \leq 10$.

Both the partial and the total photoionization cross-sections are obtained for all bound levels using the BPRM $R$-matrix codes (Berrington et al., 1995; Nahar and Pradhan, 1994; Zhang et al., 1999). They are extensions of the Opacity Project codes (Berrington et al., 1987) to include the relativistic effects (Scott and Burke, 1980; Scott and Taylor, 1982). The energy levels are obtained from STGB and are identified using the code PRCBPID (Nahar and Pradhan, 2000). 


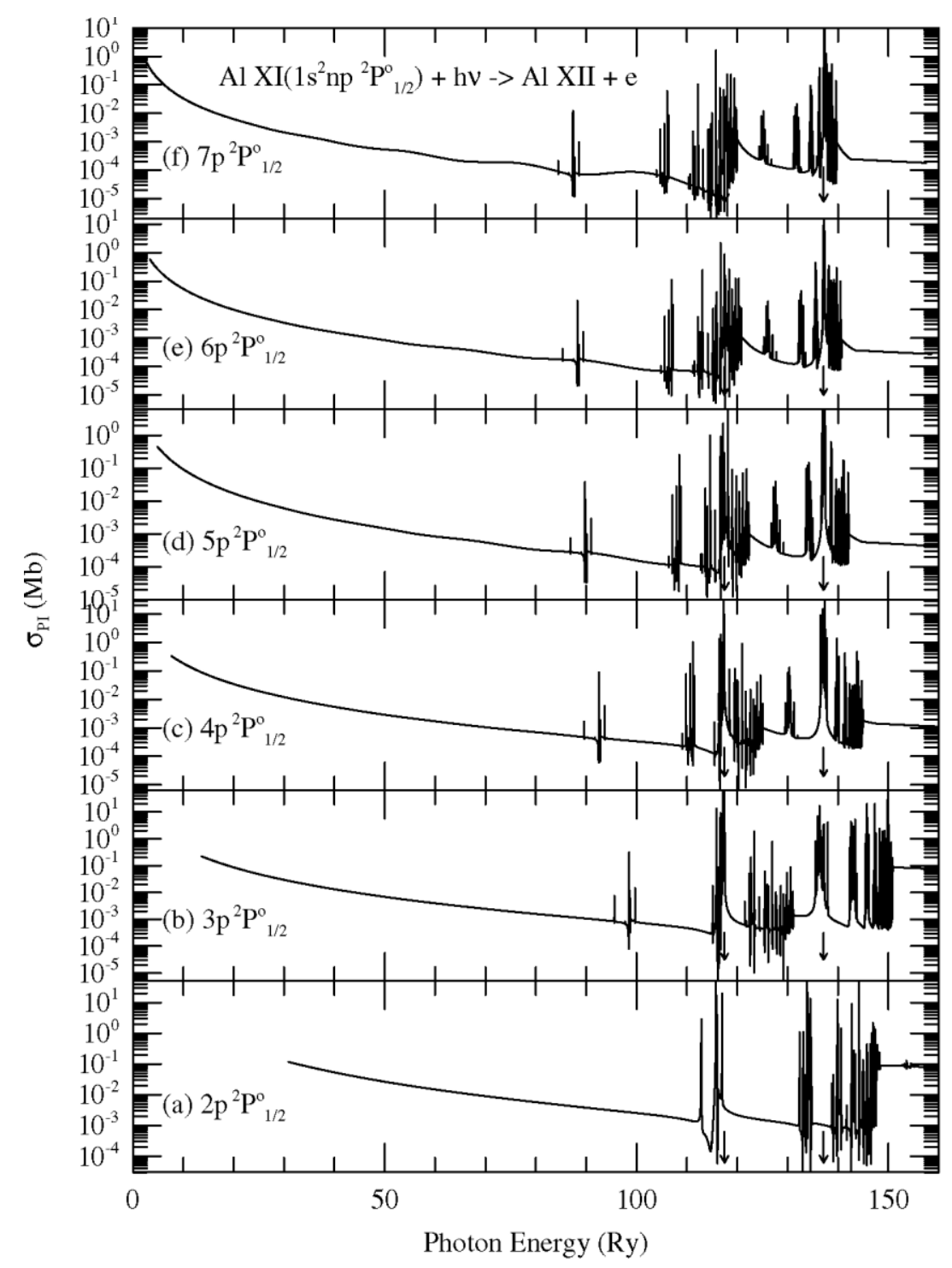

Fig. 3. Total photoionization cross-sections of the excited Rydberg series of levels, $1 s^{2} n p\left({ }^{2} \mathrm{P}_{1 / 2}^{0}\right)$ with $2 \leq \mathrm{n} \leq 7$, of $\mathrm{Al}$ XI. Positions of prominent PEC (photoexcitation-of-core) resonances are pointed by arrows at about 117 Ry due to core excitation to $\mathrm{n}=2$ levels $1 \mathrm{~s} 2 \mathrm{p}^{3} \mathrm{P}_{i}^{0},{ }_{1}^{1} \mathrm{P}_{1}^{\circ}$ and at about 137 Ry to $\mathrm{n}=3$ levels $1 \mathrm{~s} 3 \mathrm{p}^{3} \mathrm{P}_{1}^{0},{ }_{1}^{1} \mathrm{P}_{1}^{0}$. 
Table 3

Level specific recombination rate coefficients for the ground and excited $2 l$ and $3 l$ levels of Al XI and Si XII

\begin{tabular}{|c|c|c|c|c|c|c|c|c|}
\hline \multirow[t]{2}{*}{$\log _{10} T(\mathrm{~K})$} & \multicolumn{8}{|c|}{$\alpha_{\mathrm{k}}\left(\mathrm{cm}^{3} \mathrm{~s}^{-1}\right)$} \\
\hline & $2 s^{2} s_{1 / 2}$ & $3 s^{2} S_{1 / 2}$ & $2 \mathrm{p}^{2} \mathrm{P}_{1 / 2}^{\mathrm{o}}$ & $3 \mathrm{p}^{2} \mathrm{P}_{1 / 2}^{0}$ & $3 \mathrm{~d}^{2} D_{3 / 2}$ & $2 \mathrm{p}^{2} \mathrm{P}_{3 / 2}^{\mathrm{o}}$ & $3 \mathrm{p}^{2} \mathrm{P}_{3 / 2}^{\mathrm{o}}$ & $3 \mathrm{~d}^{2} D_{5 / 2}$ \\
\hline & \multicolumn{8}{|l|}{$\Lambda I X I$} \\
\hline BE: & -32.5 & -14.1 & -30.9 & -13.6 & -13.5 & -30.8 & -13.6 & -13.5 \\
\hline 1.0 & $9.35 E-11$ & $2.98 \mathrm{E}-11$ & $9.23 \mathrm{E}-11$ & $3.40 \mathrm{E}-11$ & $4.51 \mathrm{E}-11$ & $1.84 \mathrm{E}-10$ & $6.78 \mathrm{E}-11$ & $6.74 \mathrm{E}-11$ \\
\hline 1.1 & $8.33 \mathrm{E}-11$ & $2.66 \mathrm{E}-11$ & $8.23 \mathrm{E}-11$ & $3.03 E-11$ & $4.02 \mathrm{E}-11$ & $1.64 \mathrm{E}-10$ & $6.04 \mathrm{E}-11$ & $6.01 \mathrm{E}-11$ \\
\hline 1.2 & $7.43 \mathrm{E}-11$ & $2.37 \mathrm{E}-11$ & $7.34 \mathrm{E} \quad 11$ & $2.70 \mathrm{E}-11$ & $3.58 \mathrm{E}-11$ & $1.46 \mathrm{E}-10$ & $5.38 \mathrm{E}-11$ & $5.36 \mathrm{E}-11$ \\
\hline 1.3 & $6.62 \mathrm{E}-11$ & $2.11 \mathrm{E}-11$ & $6.54 \mathrm{E}-11$ & $2.41 \mathrm{E}-11$ & $3.19 E-11$ & $1.30 E-10$ & $4.80 \mathrm{E}-11$ & $4.77 \mathrm{E}-11$ \\
\hline 1.4 & $5.90 \mathrm{E}-11$ & $1.88 \mathrm{E}-11$ & $5.83 \mathrm{E}-11$ & $2.14 \mathrm{E}-11$ & $2.84 \mathrm{E} \quad 11$ & $1.16 \mathrm{E} \quad 10$ & $4.28 \mathrm{E} \quad 11$ & $\begin{array}{lll}4.25 \mathrm{E} & 11\end{array}$ \\
\hline 1.5 & $5.26 \mathrm{E}-11$ & $1.68 \mathrm{E}-11$ & $5.19 \mathrm{E}-11$ & $1.91 \mathrm{E}-11$ & $2.53 \mathrm{E}-11$ & $1.03 E-10$ & $3.81 \mathrm{E}-11$ & $3.79 \mathrm{E}-11$ \\
\hline 1.6 & $4.69 \mathrm{E}-11$ & $1.49 \mathrm{E}-11$ & $4.63 \mathrm{E}-11$ & $1.70 \mathrm{E}-11$ & $2.26 \mathrm{E}-11$ & $9.20 \mathrm{E}-11$ & $3.40 \mathrm{E} \quad 11$ & $3.38 \mathrm{E}-11$ \\
\hline 1.7 & $4.18 \mathrm{E}-11$ & $1.33 E-11$ & $4.12 E-11$ & $1.52 \mathrm{E}-11$ & $2.01 \mathrm{E} \quad 11$ & $8.20 \mathrm{E} \quad 11$ & $3.03 E-11$ & $3.01 E-11$ \\
\hline 1.8 & $3.72 \mathrm{E}-11$ & $1.19 \mathrm{E}-11$ & $3.68 \mathrm{E}-11$ & $1.35 \mathrm{E}-11$ & $1.79 \mathrm{E}-11$ & $7.31 \mathrm{E}-11$ & $2.70 \mathrm{E}-11$ & $2.68 \mathrm{E}-11$ \\
\hline 1.9 & $3.32 \mathrm{E}-11$ & $1.06 \mathrm{E}-11$ & $3.28 \mathrm{E}-11$ & $1.21 \mathrm{E}-11$ & $1.60 \mathrm{E}-11$ & $6.51 \mathrm{E}-11$ & $2.41 E-11$ & $2.39 \mathrm{E}-11$ \\
\hline 2.0 & $2.96 \mathrm{E}-11$ & $9.43 E-12$ & $2.92 E-11$ & $1.07 \mathrm{E}-11$ & $1.42 E-11$ & $5.81 \mathrm{E}-11$ & $2.14 \mathrm{E}-11$ & $2.13 E-11$ \\
\hline 2.1 & $2.64 \mathrm{E}-11$ & $8.40 \mathrm{E} \quad 12$ & $2.60 \mathrm{E} \quad 11$ & $9.57 \mathrm{E}-12$ & $1.27 \mathrm{E} \quad 11$ & $5.17 \mathrm{E}-11$ & $1.91 \mathrm{E} \quad 11$ & $1.90 \mathrm{E} \quad 11$ \\
\hline 2.2 & $2.35 \mathrm{E}-11$ & $7.49 \mathrm{E} \quad 12$ & $2.32 \mathrm{E}-11$ & $8.53 \mathrm{E}-12$ & $1.13 \mathrm{E}-11$ & $4.61 \mathrm{E}-11$ & $1.70 \mathrm{E}-11$ & $1.69 \mathrm{E} \quad 11$ \\
\hline 2.3 & $2.09 E-11$ & 6.68E $\quad 12$ & $2.07 \mathrm{E} \quad 11$ & $7.61 \mathrm{E}-12$ & $1.01 \mathrm{E}-11$ & $4.11 \mathrm{E}-11$ & $1.52 \mathrm{E} \quad 11$ & 1.51 E 11 \\
\hline 2.4 & $1.87 \mathrm{E}-11$ & $5.95 \mathrm{E}-12$ & $1.84 \mathrm{E}-11$ & $6.78 \mathrm{E}-12$ & 8.99 E $\quad 12$ & $3.66 \mathrm{E}-11$ & $1.35 \mathrm{E} \quad 11$ & $1.35 \mathrm{E}-11$ \\
\hline 2.5 & $1.66 \mathrm{E}-11$ & $5.30 \mathrm{E}-12$ & $1.64 \mathrm{E}-11$ & $6.04 \mathrm{E}-12$ & $8.01 \mathrm{E}-12$ & $3.26 \mathrm{E} \quad 11$ & $1.21 \mathrm{E}-11$ & $1.20 \mathrm{E} \quad 11$ \\
\hline 2.6 & $1.48 \mathrm{E}-11$ & $4.73 E-12$ & $1.46 \mathrm{E}-11$ & $5.38 \mathrm{E}-12$ & $7.14 \mathrm{E}-12$ & $2.91 \mathrm{E}-11$ & $1.07 \mathrm{E}-11$ & $1.07 \mathrm{E}-11$ \\
\hline 2.7 & $1.32 \mathrm{E} \quad 11$ & $4.21 \mathrm{E} \quad 12$ & $1.30 \mathrm{E}-11$ & $\begin{array}{lll}4.80 \mathrm{E} & 12\end{array}$ & 6.36E $\quad 12$ & $2.59 \mathrm{E} \quad 11$ & $9.57 \mathrm{E} \quad 12$ & $9.52 \mathrm{E} \quad 12$ \\
\hline 2.8 & $1.18 \mathrm{E}-11$ & $3.75 E-12$ & $1.16 \mathrm{E}-11$ & $4.28 \mathrm{E}-12$ & $5.67 \mathrm{E}-12$ & $2.31 \mathrm{E}-11$ & $8.53 E-12$ & $8.49 \mathrm{E}-12$ \\
\hline 2.9 & $1.05 \mathrm{E}-11$ & $3.35 E-12$ & $1.04 \mathrm{E} \quad 11$ & $3.81 \mathrm{E}-12$ & $\begin{array}{ll}5.05 \mathrm{E} & 12\end{array}$ & 2.06E $\quad 11$ & $7.60 \mathrm{E}-12$ & $7.56 \mathrm{E}-12$ \\
\hline 3.0 & $\begin{array}{ll}9.35 \mathrm{E} & 12\end{array}$ & $2.98 \mathrm{E}-12$ & $9.23 \mathrm{E}-12$ & $3.40 \mathrm{E}-12$ & $4.50 \mathrm{E}-12$ & $1.84 \mathrm{E}-11$ & $6.78 \mathrm{E}-12$ & $6.74 \mathrm{E}-12$ \\
\hline 3.1 & $8.34 \mathrm{E}-12$ & $2.66 \mathrm{E}-12$ & $8.23 E-12$ & $3.03 E-12$ & $4.01 \mathrm{E}-12$ & $1.64 \mathrm{E}-11$ & $6.04 E-12$ & $6.00 \mathrm{E} \quad 12$ \\
\hline 3.2 & $7.43 E-12$ & $2.37 \mathrm{E}-12$ & $7.33 E-12$ & $2.70 \mathrm{E}-12$ & $3.57 \mathrm{E}-12$ & $1.4 \mathrm{GE}-11$ & $5.38 \mathrm{E}-12$ & $5.35 \mathrm{E}-12$ \\
\hline 3.3 & $6.62 \mathrm{E}-12$ & $2.11 \mathrm{E}-12$ & $6.54 \mathrm{E}-12$ & $2.40 \mathrm{E}-12$ & $3.19 \mathrm{E}-12$ & $1.30 \mathrm{E}-11$ & $4.80 E-12$ & $4.77 \mathrm{E}-12$ \\
\hline 3.4 & $5.90 E-12$ & $1.88 \mathrm{E}-12$ & $5.82 \mathrm{E}-12$ & $2.14 \mathrm{E}-12$ & $2.84 \mathrm{E}-12$ & $1.16 \mathrm{E}-11$ & $4.28 \mathrm{E}-12$ & $4.25 \mathrm{E}-12$ \\
\hline 3.5 & $5.26 \mathrm{E}-12$ & $1.68 \mathrm{E}-12$ & $\begin{array}{lll}5.19 E & 12\end{array}$ & $1.91 \mathrm{E} \quad 12$ & $2.53 E-12$ & $1.03 E-11$ & $3.81 \mathrm{E}-12$ & $3.78 \mathrm{E}-12$ \\
\hline 3.6 & $4.69 \mathrm{E} \quad 12$ & $1.49 \mathrm{E}-12$ & $4.63 \mathrm{E}-12$ & $1.70 \mathrm{E}-12$ & $2.25 \mathrm{E} \quad 12$ & $9.19 \mathrm{E}-12$ & $3.39 \mathrm{E} \quad 12$ & $3.37 \mathrm{E}-12$ \\
\hline 3.7 & $4.18 \mathrm{E}-12$ & $1.33 \mathrm{E}-12$ & $\begin{array}{lll}4.12 E & 12\end{array}$ & $1.52 \mathrm{E}-12$ & $2.01 \mathrm{E} \quad 12$ & $\begin{array}{ll}8.19 E & 12\end{array}$ & $3.03 E-12$ & $3.00 \mathrm{E} \quad 12$ \\
\hline 3.8 & $3.72 E-12$ & $1.19 E-12$ & $3.67 \mathrm{E}-12$ & $1.35 E-12$ & $1.79 E-12$ & $7.30 \mathrm{E}-12$ & $2.70 \mathrm{E}-12$ & $2.67 \mathrm{E}-12$ \\
\hline 3.9 & $3.32 \mathrm{E}-12$ & $1.06 \mathrm{E}-12$ & $3.27 \mathrm{E}-12$ & $1.20 \mathrm{E}-12$ & $1.59 \mathrm{E} \quad 12$ & $\begin{array}{lll}6.50 \mathrm{E} & 12\end{array}$ & $2.40 \mathrm{E}-12$ & $2.38 \mathrm{E} \quad 12$ \\
\hline 4.0 & $2.96 \mathrm{E}-12$ & $9.43 E-13$ & $2.92 E-12$ & $1.07 \mathrm{E}-12$ & $1.41 \mathrm{E}-12$ & $5.79 \mathrm{E}-12$ & $2.14 E-12$ & $2.12 \mathrm{E}-12$ \\
\hline 4.1 & $2.64 \mathrm{E}-12$ & $8.41 \mathrm{E}-13$ & $2.60 \mathrm{E}-12$ & $9.55 \mathrm{E}-13$ & $1.26 \mathrm{E}-12$ & $5.16 \mathrm{E}-12$ & $1.91 \mathrm{E}-12$ & $1.88 \mathrm{E}-12$ \\
\hline 4.2 & $2.35 \mathrm{E}-12$ & $7.49 \mathrm{E}-13$ & $2.31 \mathrm{E} \quad 12$ & $8.51 \mathrm{E}-13$ & $\begin{array}{lll}1.12 E & 12\end{array}$ & $\begin{array}{ll}4.60 \mathrm{E} & 12\end{array}$ & $1.70 \mathrm{E} \quad 12$ & $1.68 \mathrm{E} \quad 12$ \\
\hline 4.3 & $2.10 \mathrm{E}-12$ & $6.68 \mathrm{E}-13$ & $2.06 \mathrm{E}-12$ & $7.58 \mathrm{E}-13$ & $9.95 \mathrm{E}-13$ & $4.09 \mathrm{E}-12$ & $1.51 \mathrm{E}-12$ & $1.49 \mathrm{E}-12$ \\
\hline 4.4 & $1.87 \mathrm{E}-12$ & $\begin{array}{lll}5.95 E & 13\end{array}$ & $1.83 \mathrm{E}-12$ & $6.75 E-13$ & $8.84 \mathrm{E} \quad 13$ & $3.64 \mathrm{E} \quad 12$ & $\begin{array}{lll}1.35 \mathrm{E} & 12\end{array}$ & $\begin{array}{lll}1.32 \mathrm{E} & 12\end{array}$ \\
\hline 4.5 & $1.66 \mathrm{E}-12$ & $5.31 \mathrm{E}-13$ & $1.63 \mathrm{E}-12$ & $6.01 \mathrm{E}-13$ & $7.85 \mathrm{E}-13$ & $3.24 \mathrm{E}-12$ & $1.20 \mathrm{E}-12$ & $1.17 \mathrm{E} \quad 12$ \\
\hline 4.6 & $1.48 \mathrm{E}-12$ & $4.73 E-13$ & $1.45 \mathrm{E}-12$ & $5.35 E-13$ & $6.96 E-13$ & $2.89 \mathrm{E}-12$ & $1.07 \mathrm{E}-12$ & $1.04 \mathrm{E}-12$ \\
\hline 4.7 & $1.32 \mathrm{E}-12$ & $4.22 \mathrm{E}-13$ & $1.29 \mathrm{E}-12$ & $4.76 E-13$ & $6.16 E-13$ & $2.57 \mathrm{E}-12$ & $9.49 E-13$ & $\begin{array}{lll}9.22 \mathrm{E} & -13\end{array}$ \\
\hline 4.8 & $1.18 \mathrm{E}-12$ & $3.76 \mathrm{E} \quad 13$ & $1.15 E-12$ & $4.23 E-13$ & $5.44 \mathrm{E} \quad 13$ & $2.28 \mathrm{E}-12$ & $8.44 E-13$ & $8.15 \mathrm{E} \quad 13$ \\
\hline 4.9 & $1.05 \mathrm{E}-12$ & $3.36 \mathrm{E}-13$ & $1.02 \mathrm{E}-12$ & $3.76 E-13$ & $4.80 E-13$ & $2.03 E-12$ & $7.50 \mathrm{E}-13$ & $7.19 \mathrm{E}-13$ \\
\hline 5.0 & $9.38 \mathrm{E}-13$ & $2.99 \mathrm{E}-13$ & $9.06 \mathrm{E}-13$ & $3.34 \mathrm{E}-13$ & $4.23 \mathrm{E}-13$ & $1.80 \mathrm{E}-12$ & $6.66 E-13$ & $6.33 E-13$ \\
\hline 5.1 & $8.36 \mathrm{E}-13$ & $2.67 \mathrm{E}-13$ & $8.03 E-13$ & $2.96 \mathrm{E}-13$ & $3.71 \mathrm{E}-13$ & $1.60 \mathrm{E}-12$ & $5.90 \mathrm{E}-13$ & $\begin{array}{lll}5.55 \mathrm{E} & 13\end{array}$ \\
\hline 5.2 & $7.46 \mathrm{E}-13$ & $2.38 \mathrm{E}-13$ & $7.11 \mathrm{E}-13$ & $2.62 E-13$ & $3.25 \mathrm{E}-13$ & $1.41 \mathrm{E}-12$ & $5.23 E-13$ & $4.86 \mathrm{E}-13$ \\
\hline 5.3 & $6.65 E-13$ & $2.12 E-13$ & $6.29 \mathrm{E}-13$ & $2.32 \mathrm{E}-13$ & $2.83 E-13$ & $1.25 \mathrm{E}-12$ & $4.63 E-13$ & $4.23 E-13$ \\
\hline 5.4 & $5.94 \mathrm{E}-13$ & $1.90 \mathrm{E}-13$ & $\begin{array}{lll}5.56 E & 13\end{array}$ & $2.05 E-13$ & $2.45 E-13$ & $1.10 \mathrm{E}-12$ & $4.09 E-13$ & $3.67 \mathrm{E}-13$ \\
\hline 5.5 & $5.30 \mathrm{E}-13$ & $1.69 \mathrm{E} \quad 13$ & $\begin{array}{lll}4.89 E & 13\end{array}$ & $1.81 \mathrm{E}-13$ & $2.11 \mathrm{E} \quad 13$ & $9.72 E \quad 13$ & $3.60 \mathrm{E} \quad 13$ & $\begin{array}{lll}3.16 \mathrm{E} & 13\end{array}$ \\
\hline 5.6 & $4.73 E-13$ & $1.51 \mathrm{E}-13$ & $4.30 E-13$ & $1.59 \mathrm{E}-13$ & $1.81 \mathrm{E}-13$ & $8.54 \mathrm{E}-13$ & $3.17 \mathrm{E}-13$ & $2.71 \mathrm{E}-13$ \\
\hline 5.7 & $4.22 \mathrm{E}-13$ & $1.35 E-13$ & $3.77 \mathrm{E}-13$ & $1.39 \mathrm{E}-13$ & $1.54 \mathrm{E}-13$ & $7.48 \mathrm{E}-13$ & $2.78 \mathrm{E}-13$ & $2.31 \mathrm{E}-13$ \\
\hline 5.8 & $3.76 \mathrm{E}-13$ & $1.20 \mathrm{E}-13$ & $3.29 \mathrm{E}-13$ & $1.22 \mathrm{E}-13$ & $1.30 \mathrm{E}-13$ & $6.53 E-13$ & $2.42 E-13$ & $1.95 \mathrm{E}-13$ \\
\hline 5.9 & $3.36 E-13$ & $1.07 \mathrm{E} \quad 13$ & $2.86 \mathrm{E}-13$ & $1.06 \mathrm{E} \quad 13$ & $1.09 \mathrm{E} \quad 13$ & $5.67 \mathrm{E} \quad 13$ & $2.11 \mathrm{E} \quad 13$ & $1.63 \mathrm{E} \quad 13$ \\
\hline 6.0 & $2.99 \mathrm{E}-13$ & $\begin{array}{ccc}9.53 E & 14\end{array}$ & $2.47 \mathrm{E}-13$ & 9.15 E $\quad 14$ & $\begin{array}{lll}9.06 \mathrm{E} & 14\end{array}$ & $4.90 \mathrm{E} \quad 13$ & $1.82 \mathrm{E} \quad 13$ & $1.35 \mathrm{E} \quad 13$ \\
\hline 6.1 & $2.67 \mathrm{E}-13$ & $8.48 \mathrm{E}-14$ & $2.13 E-13$ & $7.87 \mathrm{E}-14$ & $7.44 \mathrm{E}-14$ & $4.22 \mathrm{E}-13$ & $1.57 \mathrm{E}-13$ & $1.11 \mathrm{E}-13$ \\
\hline 6.2 & $2.38 \mathrm{E}-13$ & $7.55 E-14$ & $1.83 \mathrm{E}-13$ & $6.74 \mathrm{E}-14$ & $6.07 \mathrm{E}-14$ & $3.62 \mathrm{E}-13$ & $1.35 \mathrm{E}-13$ & $9.06 \mathrm{E}-14$ \\
\hline 6.3 & $2.15 \mathrm{E}-13$ & $\begin{array}{lll}6.78 \mathrm{E} & 14\end{array}$ & $1.59 \mathrm{E}-13$ & $5.81 \mathrm{E}-14$ & $4.95 \mathrm{E}-14$ & $3.15 \mathrm{E}-13$ & $1.18 \mathrm{E} \quad 13$ & $7.31 \mathrm{E} \quad 14$ \\
\hline 6.4 & $1.98 \mathrm{E}-13$ & $6.29 E-14$ & $1.45 \mathrm{E}-13$ & $5.21 \mathrm{E}-14$ & $4.17 \mathrm{E}-14$ & $2.87 \mathrm{E}-13$ & $1.12 E-13$ & $5.88 \mathrm{E}-14$ \\
\hline 6.5 & $1.90 \mathrm{E}-13$ & $6.25 \mathrm{E}-14$ & $1.42 \mathrm{E}-13$ & $5.11 \mathrm{E}-14$ & $3.83 \mathrm{E}-14$ & $2.81 \mathrm{E}-13$ & $1.25 \mathrm{E}-13$ & $4.76 \mathrm{E}-14$ \\
\hline 6.6 & $1.88 \mathrm{E}-13$ & $6.71 \mathrm{E}-14$ & $1.50 \mathrm{E}-13$ & $5.58 \mathrm{E}-14$ & $3.97 \mathrm{E}-14$ & $2.94 \mathrm{E}-13$ & $1.58 \mathrm{E}-13$ & $3.95 \mathrm{E}-14$ \\
\hline 6.7 & $1.90 \mathrm{E}-13$ & $7.51 \mathrm{E}-14$ & $1.62 \mathrm{E}-13$ & $6.46 E-14$ & $4.46 \mathrm{E}-14$ & $3.18 \mathrm{E}-13$ & $2.06 \mathrm{E}-13$ & $3.40 \mathrm{E}-14$ \\
\hline 6.8 & $1.91 \mathrm{E}-13$ & $8.34 \mathrm{E}-14$ & $1.72 \mathrm{E}-13$ & $7.40 \mathrm{E}-14$ & $\begin{array}{lll}5.05 E & 14\end{array}$ & $\begin{array}{lll}3.38 \mathrm{E} & -13\end{array}$ & $2.55 \mathrm{E}-13$ & $3.00 \mathrm{E}-14$ \\
\hline 6.9 & $1.86 \mathrm{E}-13$ & $8.86 \mathrm{E}-14$ & $1.75 \mathrm{E}-13$ & $8.04 \mathrm{E}-14$ & $\begin{array}{lll}5.47 \mathrm{E} & 14\end{array}$ & $3.44 \mathrm{E}-13$ & $2.90 \mathrm{E}-13$ & $2.69 E-14$ \\
\hline 7.0 & $1.75 \mathrm{E}-13$ & $8.89 \mathrm{E}-14$ & $1.69 \mathrm{E}-13$ & $\begin{array}{lll}8.20 \mathrm{E} & 14\end{array}$ & $5.57 \mathrm{E} \quad 14$ & $\begin{array}{lll}3.32 \mathrm{E} & 13\end{array}$ & $3.03 \mathrm{E}-13$ & $2.39 \mathrm{E} \quad 14$ \\
\hline 7.1 & $1.59 \mathrm{E}-13$ & $8.44 \mathrm{E}-14$ & $1.55 \mathrm{E}-13$ & $\begin{array}{ll}7.85 E & 14\end{array}$ & $5.34 \mathrm{E}-14$ & $3.04 E-13$ & $2.95 \mathrm{E} \quad 13$ & $2.09 \mathrm{E} \quad 14$ \\
\hline 7.2 & $1.39 \mathrm{E}-13$ & $7.62 E-14$ & $1.36 \mathrm{E}-13$ & $7.11 \mathrm{E}-14$ & $4.83 \mathrm{E}-14$ & $2.67 E-13$ & $2.70 E-13$ & $1.77 \mathrm{E}-14$ \\
\hline 7.3 & $1.18 \mathrm{E}-13$ & $6.58 \mathrm{E}-14$ & $1.14 \mathrm{E}-13$ & $6.14 E-14$ & $4.17 \mathrm{E}-14$ & $2.25 E-13$ & $2.35 E-13$ & $1.47 \mathrm{E}-14$ \\
\hline 7.4 & $9.72 \mathrm{E}-14$ & $5.49 \mathrm{E}-14$ & $9.34 \mathrm{E}-14$ & $5.11 \mathrm{E}-14$ & $3.47 \mathrm{E}-14$ & $1.83 \mathrm{E}-13$ & $1.96 \mathrm{E}-13$ & $1.18 \mathrm{E}-14$ \\
\hline 7.5 & $7.85 \mathrm{E}-14$ & $4.45 \mathrm{E}-14$ & $7.42 E-14$ & $4.11 \mathrm{E}-14$ & $2.79 \mathrm{E}-14$ & $1.46 \mathrm{E}-13$ & $1.58 \mathrm{E}-13$ & $9.31 \mathrm{E}-15$ \\
\hline 7.6 & $6.23 E-14$ & $3.52 E-14$ & $5.77 \mathrm{E}-14$ & $3.23 E-14$ & $2.19 \mathrm{E}-14$ & $1.13 E-13$ & $1.24 \mathrm{E}-13$ & $7.20 \mathrm{E}-15$ \\
\hline 7.7 & $\begin{array}{lll}4.87 \mathrm{E} & 14\end{array}$ & $2.74 \mathrm{E}-14$ & $4.41 \mathrm{E}-14$ & $2.49 \mathrm{E} \quad 14$ & $1.68 \mathrm{E} \quad 14$ & $8.66 \mathrm{E} \quad 14$ & $9.58 \mathrm{E} \quad 14$ & 5.47 E 15 \\
\hline 7.8 & $3.76 \mathrm{E}-14$ & $2.09 \mathrm{E} \quad 14$ & $3.33 E-14$ & $1.89 \mathrm{E}-14$ & $1.27 \mathrm{E} \quad 14$ & $6.53 \mathrm{E}-14$ & $7.26 \mathrm{E}-14$ & $4.11 \mathrm{E}-15$ \\
\hline 7.9 & $2.88 \mathrm{E}-14$ & $1.58 \mathrm{E}-14$ & $2.48 \mathrm{E}-14$ & $1.41 \mathrm{E}-14$ & $9.48 \mathrm{E} \quad 15$ & $4.87 \mathrm{E}-14$ & $5.42 \mathrm{E}-14$ & $3.05 \mathrm{E}-15$ \\
\hline 8.0 & $2.19 \mathrm{E}-14$ & $1.19 \mathrm{E} \quad 14$ & $1.83 \mathrm{E}-14$ & $1.04 \mathrm{E}-14$ & $7.00 \mathrm{E}-15$ & $3.60 \mathrm{E} \quad 14$ & $4.01 \mathrm{E} \quad 14$ & $2.24 \mathrm{E} \quad 15$ \\
\hline 8.1 & $1.65 E-14$ & $8.82 \mathrm{E}-15$ & $1.35 \mathrm{E}-14$ & $7.67 \mathrm{E}-15$ & $5.12 \mathrm{E} \quad 15$ & $2.64 \mathrm{E} \quad 14$ & $2.94 \mathrm{E}-14$ & $1.64 \mathrm{E}-15$ \\
\hline
\end{tabular}


Table 3 (continued)

\begin{tabular}{|c|c|c|c|c|c|c|c|c|}
\hline \multirow[t]{2}{*}{$\log _{10} T(\mathrm{~K})$} & \multicolumn{8}{|c|}{$\alpha_{\mathrm{k}}\left(\mathrm{cm}^{3} \mathrm{~s}^{-1}\right)$} \\
\hline & $2 s^{2} S_{1 / 2}$ & $3 s^{2} s_{1 / 2}$ & $2 \mathrm{p}^{2} \mathrm{P}_{1 / 2}^{0}$ & $3 \mathrm{p}^{2} \mathrm{P}_{1 / 2}^{0}$ & $3 \mathrm{~d}^{2} D_{3 / 2}$ & $2 \mathrm{p}^{2} \mathrm{P}_{3 / 2}^{0}$ & $3 \mathrm{p}^{2} \mathrm{P}_{3 / 2}^{0}$ & $3 d^{2} D_{3 i 2}$ \\
\hline \multirow[b]{2}{*}{ BE: } & \multicolumn{8}{|l|}{$\mathrm{Al} \mathrm{XI}$} \\
\hline & 32.5 & 14.1 & -30.9 & 13.6 & -13.5 & -30.8 & -13.6 & -13.5 \\
\hline 8.2 & $1.24 \mathrm{E} \quad 14$ & $6.52 E-15$ & $9.83 E-15$ & $5.59 \mathrm{E}-15$ & $3.72 \mathrm{E} \quad 15$ & $1.93 \mathrm{E} \quad 14$ & $2.14 \mathrm{E}-14$ & $1.19 \mathrm{E}-15$ \\
\hline 8.3 & $9.26 \mathrm{E}-15$ & $4.80 \mathrm{E}-15$ & $7.15 \mathrm{E}-15$ & $4.06 \mathrm{E}-15$ & $2.69 \mathrm{E}-15$ & $1.40 \mathrm{E}-14$ & $1.55 \mathrm{E}-14$ & $8.63 E-16$ \\
\hline 8.4 & 6.90E 15 & $3.51 \mathrm{E} \quad 15$ & $5.18 \mathrm{E}-15$ & $2.94 \mathrm{E} \quad 15$ & $1.94 \mathrm{E} \quad 15$ & $1.02 \mathrm{E}-14$ & $1.12 \mathrm{E}-14$ & $6.22 \mathrm{E}-1 \mathrm{G}$ \\
\hline 8.5 & $5.12 \mathrm{E}-15$ & $2.57 \mathrm{E}-15$ & $3.74 \mathrm{E}-15$ & $2.12 \mathrm{E}-15$ & $1.39 \mathrm{E}-15$ & $7.34 \mathrm{E}-15$ & $8.05 E-15$ & $4.47 \mathrm{E}-16$ \\
\hline 8.6 & $3.79 E-15$ & $1.87 \mathrm{E}-15$ & $2.70 \mathrm{E}-15$ & $1.52 \mathrm{E}-15$ & $9.97 \mathrm{E}-16$ & $5.29 \mathrm{E}-15$ & $5.78 \mathrm{E}-15$ & $3.21 \mathrm{E}-16$ \\
\hline 8.7 & $2.80 \mathrm{E}-15$ & $1.36 \mathrm{E} \quad 15$ & $1.94 \mathrm{E}-15$ & 1.09 E $\quad 15$ & $\begin{array}{lll}7.12 E & 16\end{array}$ & $3.81 \mathrm{E}-15$ & $4.13 \mathrm{E}-15$ & $2.29 \mathrm{E}-16$ \\
\hline 8.8 & $2.07 \mathrm{E}-15$ & $9.89 \mathrm{E}-16$ & $1.40 \mathrm{E}-15$ & $7.81 \mathrm{E}-16$ & $5.08 \mathrm{E}-16$ & $2.74 \mathrm{E}-15$ & $2.95 E-15$ & $1.64 \mathrm{E}-16$ \\
\hline 8.9 & $1.52 \mathrm{E}-15$ & $7.17 \mathrm{E}-16$ & $1.00 \mathrm{E}-15$ & $5.59 \mathrm{E}-16$ & $3.62 E-16$ & $1.97 \mathrm{E}-15$ & $2.11 \mathrm{E}-15$ & $1.17 \mathrm{E}-16$ \\
\hline \multirow[t]{2}{*}{9.0} & $1.12 \mathrm{E}-15$ & $5.19 \mathrm{E}-16$ & $7.18 \mathrm{E}-16$ & $3.99 \mathrm{E}-16$ & $2.57 \mathrm{E}-16$ & $1.41 \mathrm{E}-15$ & $1.50 \mathrm{E}-15$ & $8.36 \mathrm{E}-17$ \\
\hline & \multicolumn{8}{|l|}{ Si XII } \\
\hline BE: & -38.5 & 16.7 & -36.7 & -16.2 & 16.0 & -36.6 & -16.2 & -16.0 \\
\hline 1.0 & $1.10 \mathrm{E}-10$ & $3.53 \mathrm{E}-11$ & $1.10 \mathrm{E}-10$ & $4.04 \mathrm{E}-11$ & $5.36 \mathrm{E}-11$ & $2.18 \mathrm{E}-10$ & $8.04 \mathrm{E}-11$ & $8.02 \mathrm{E}-11$ \\
\hline 1.1 & $9.77 \mathrm{E}-11$ & $3.14 \mathrm{E}-11$ & $9.77 \mathrm{E}-11$ & $3.60 \mathrm{E}-11$ & $4.78 \mathrm{E}-11$ & $1.94 \mathrm{E}-10$ & $7.17 \mathrm{E}-11$ & $7.15 \mathrm{E}-11$ \\
\hline 1.2 & $8.71 \mathrm{E}-11$ & $2.80 \mathrm{E}-11$ & $8.71 \mathrm{E}-11$ & $3.21 \mathrm{E}-11$ & $4.26 \mathrm{E}-11$ & $1.73 \mathrm{E}-10$ & $6.39 E-11$ & $6.37 \mathrm{E}-11$ \\
\hline 1.3 & $7.76 \mathrm{E}-11$ & $2.50 \mathrm{E}-11$ & $7.76 \mathrm{E}-11$ & $2.86 \mathrm{E}-11$ & $3.79 \mathrm{E}-11$ & $1.54 \mathrm{E}-10$ & $5.69 \mathrm{E}-11$ & $5.68 \mathrm{E}-11$ \\
\hline 1.4 & $6.92 \mathrm{E}-11$ & $2.22 \mathrm{E}-11$ & $6.92 \mathrm{E}-11$ & $2.55 \mathrm{E}-11$ & $3.38 \mathrm{E}-11$ & $1.37 \mathrm{E}-10$ & $5.08 \mathrm{E}-11$ & $5.06 \mathrm{E}-11$ \\
\hline 1.5 & $6.16 \mathrm{E}-11$ & $1.98 \mathrm{E} \quad 11$ & $6.16 \mathrm{E}-11$ & $2.27 \mathrm{E} \quad 11$ & $3.01 \mathrm{E} \quad 11$ & $1.22 \mathrm{E} \quad 10$ & $4.52 \mathrm{E}-11$ & $4.51 \mathrm{E}-11$ \\
\hline 1.6 & $5.49 \mathrm{E}-11$ & $1.77 \mathrm{E}-11$ & $5.49 \mathrm{E}-11$ & $2.02 \mathrm{E}-11$ & $2.69 \mathrm{E}-11$ & $1.09 \mathrm{E}-10$ & $4.03 E-11$ & $4.02 \mathrm{E}-11$ \\
\hline 1.7 & $4.90 \mathrm{E}-11$ & $1.57 \mathrm{E}-11$ & $4.90 \mathrm{E}-11$ & $1.80 \mathrm{E}-11$ & $2.39 \mathrm{E}-11$ & $9.72 \mathrm{E}-11$ & $3.59 \mathrm{E}-11$ & $3.58 \mathrm{E}-11$ \\
\hline 1.8 & $4.36 \mathrm{E} \quad 11$ & $1.40 \mathrm{E} \quad 11$ & $4.36 \mathrm{E}-11$ & $1.61 \mathrm{E} \quad 11$ & $2.13 E-11$ & $8.66 E-11$ & $3.20 \mathrm{E}-11$ & $3.19 \mathrm{E}-11$ \\
\hline 1.9 & $3.89 \mathrm{E}-11$ & $1.25 \mathrm{E}-11$ & $3.89 \mathrm{E}-11$ & $1.43 \mathrm{E}-11$ & $1.90 \mathrm{E}-11$ & $7.72 \mathrm{E}-11$ & $2.85 \mathrm{E}-11$ & $2.84 \mathrm{E}-11$ \\
\hline 2.0 & $3.47 \mathrm{E}-11$ & $1.11 \mathrm{E} \quad 11$ & $3.47 \mathrm{E}-11$ & $1.28 \mathrm{E} \quad 11$ & $1.69 \mathrm{E}-11$ & $\begin{array}{lll}6.88 \mathrm{E} & 11\end{array}$ & $2.54 \mathrm{E}-11$ & $2.54 \mathrm{E} \quad 11$ \\
\hline 2.1 & $3.09 \mathrm{E} \quad 11$ & $9.93 \mathrm{E}-12$ & $3.09 \mathrm{E} \quad 11$ & $1.14 \mathrm{E} \quad 11$ & $1.51 \mathrm{E}-11$ & $\begin{array}{lll}6.13 \mathrm{E} & 11\end{array}$ & $2.27 \mathrm{E}-11$ & $2.26 \mathrm{E}-11$ \\
\hline 2.2 & $2.75 \mathrm{E}-11$ & $8.85 \mathrm{E}-12$ & $2.75 E-11$ & $1.01 \mathrm{E}-11$ & $1.35 E-11$ & $5.47 \mathrm{E}-11$ & $2.02 \mathrm{E}-11$ & $2.01 \mathrm{E}-11$ \\
\hline 2.3 & $2.45 E-11$ & $7.89 \mathrm{E}-12$ & $2.45 \mathrm{E}-11$ & $9.03 E-12$ & $1.20 \mathrm{E}-11$ & $4.87 \mathrm{E}-11$ & $1.80 \mathrm{E}-11$ & $1.79 \mathrm{E}-11$ \\
\hline 2.4 & $2.19 \mathrm{E}-11$ & $7.03 \mathrm{E}-12$ & $2.19 \mathrm{E}-11$ & $8.05 \mathrm{E}-12$ & $1.07 \mathrm{E}-11$ & $4.34 \mathrm{E}-11$ & $1.60 \mathrm{E}-11$ & $1.60 \mathrm{E}-11$ \\
\hline 2.5 & $1.95 \mathrm{E}-11$ & $6.27 \mathrm{E}-12$ & $1.95 \mathrm{E}-11$ & $7.18 \mathrm{E}-12$ & $9.53 \mathrm{E}-12$ & $3.87 \mathrm{E}-11$ & $1.43 \mathrm{E}-11$ & $1.43 \mathrm{E}-11$ \\
\hline 2.6 & $1.74 \mathrm{E}-11$ & $\begin{array}{lll}5.59 \mathrm{E} & 12\end{array}$ & $1.74 \mathrm{E}-11$ & $6.40 \mathrm{E} \quad 12$ & $8.49 \mathrm{E} \quad 12$ & $3.45 \mathrm{E}-11$ & $1.27 \mathrm{E}-11$ & $1.27 \mathrm{E}-11$ \\
\hline 2.7 & $1.55 \mathrm{E}-11$ & $4.98 \mathrm{E}-12$ & $1.55 \mathrm{E}-11$ & $5.70 \mathrm{E}-12$ & $7.57 \mathrm{E}-12$ & $3.07 \mathrm{E}-11$ & $1.14 \mathrm{E}-11$ & $1.13 \mathrm{E}-11$ \\
\hline 2.8 & $1.38 \mathrm{E}-11$ & $4.44 \mathrm{E}-12$ & $1.38 \mathrm{E}-11$ & $\begin{array}{lll}5.08 \mathrm{E} & 12\end{array}$ & $6.74 \mathrm{E}-12$ & $2.74 \mathrm{E}-11$ & $1.01 \mathrm{E}-11$ & $1.01 \mathrm{E}-11$ \\
\hline 2.9 & $1.23 \mathrm{E}-11$ & $3.96 \mathrm{E} \quad 12$ & $1.23 \mathrm{E}-11$ & $\begin{array}{lll}4.53 \mathrm{E} & 12\end{array}$ & $6.01 \mathrm{E}-12$ & $2.44 \mathrm{E}-11$ & $9.02 \mathrm{E}-12$ & $8.99 \mathrm{E}-12$ \\
\hline 3.0 & $1.10 \mathrm{E}-11$ & $3.53 \mathrm{E}-12$ & $1.10 \mathrm{E}-11$ & $4.03 \mathrm{E}-12$ & $5.36 \mathrm{E}-12$ & $2.18 \mathrm{E}-11$ & $8.04 \mathrm{E}-12$ & $8.01 \mathrm{E}-12$ \\
\hline 3.1 & $9.77 \mathrm{E}-12$ & $3.14 \mathrm{E}-12$ & $9.77 \mathrm{E}-12$ & $3.60 \mathrm{E} \quad 12$ & $4.77 \mathrm{E}-12$ & $1.94 \mathrm{E} \quad 11$ & 7.17E 12 & $7.14 \mathrm{E}-12$ \\
\hline 3.2 & $8.71 \mathrm{E}-12$ & $2.80 \mathrm{E}-12$ & $8.70 \mathrm{E}-12$ & $3.20 \mathrm{E}-12$ & $4.25 \mathrm{E}-12$ & $1.73 \mathrm{E}-11$ & $6.39 \mathrm{E}-12$ & $6.36 \mathrm{E}-12$ \\
\hline 3.3 & $\begin{array}{lll}7.76 E & 12\end{array}$ & $2.50 \mathrm{E}-12$ & $7.76 \mathrm{E}-12$ & $2.86 \mathrm{E}-12$ & $3.79 \mathrm{E}-12$ & $1.54 \mathrm{E}-11$ & $\begin{array}{lll}5.69 \mathrm{E} & 12\end{array}$ & $5.67 \mathrm{E}-12$ \\
\hline 3.4 & $6.92 \mathrm{E}-12$ & $2.22 \mathrm{E}-12$ & $6.91 \mathrm{E}-12$ & $2.55 \mathrm{E}-12$ & $\begin{array}{lll}3.38 \mathrm{E} & 12\end{array}$ & $1.37 \mathrm{E}-11$ & $5.07 \mathrm{E}-12$ & $5.05 E-12$ \\
\hline 3.5 & $6.16 \mathrm{E}-12$ & $1.98 \mathrm{E}-12$ & $6.16 \mathrm{E}-12$ & $2.27 \mathrm{E}-12$ & $3.01 \mathrm{E}-12$ & $1.22 \mathrm{E}-11$ & $4.52 \mathrm{E}-12$ & $4.50 \mathrm{E}-12$ \\
\hline 3.6 & $5.49 \mathrm{E}-12$ & $1.77 \mathrm{E}-12$ & $5.49 \mathrm{E}-12$ & $2.02 \mathrm{E}-12$ & $2.68 \mathrm{E}-12$ & $1.09 \mathrm{E}-11$ & $4.03 E-12$ & $4.01 \mathrm{E}-12$ \\
\hline 3.7 & $4.90 \mathrm{E}-12$ & $1.58 \mathrm{E}-12$ & $4.89 \mathrm{E}-12$ & $1.80 \mathrm{E}-12$ & $2.39 \mathrm{E}-12$ & $9.71 \mathrm{E}-12$ & $3.59 \mathrm{E}-12$ & $3.57 \mathrm{E}-12$ \\
\hline 3.8 & $4.36 \mathrm{E}-12$ & $1.40 \mathrm{E}-12$ & 4.36E 12 & $1.61 \mathrm{E} \quad 12$ & $2.13 \mathrm{E}-12$ & $8.65 \mathrm{E}-12$ & $3.20 \mathrm{E}-12$ & $3.18 \mathrm{E}-12$ \\
\hline 3.9 & $3.89 \mathrm{E}-12$ & $1.25 \mathrm{E}-12$ & $3.88 \mathrm{E}-12$ & $1.43 \mathrm{E} \quad 12$ & $1.89 \mathrm{E}-12$ & $7.71 \mathrm{E}-12$ & $2.85 \mathrm{E}-12$ & $2.83 \mathrm{E}-12$ \\
\hline 4.0 & $3.47 \mathrm{E}-12$ & $1.12 \mathrm{E}-12$ & $3.46 \mathrm{E}-12$ & $1.27 \mathrm{E}-12$ & $1.69 E-12$ & $6.87 \mathrm{E}-12$ & $2.54 \mathrm{E}-12$ & $2.52 \mathrm{E}-12$ \\
\hline 4.1 & $3.09 \mathrm{E}-12$ & $9.94 \mathrm{E}-13$ & $3.08 \mathrm{E}-12$ & $1.14 \mathrm{E}-12$ & $1.50 \mathrm{E}-12$ & $6.12 \mathrm{E}-12$ & $2.26 \mathrm{E}-12$ & $2.24 \mathrm{E}-12$ \\
\hline 4.2 & $2.75 \mathrm{E}-12$ & $8.8 \mathrm{GE}-13$ & $2.75 \mathrm{E}-12$ & $1.01 \mathrm{E}-12$ & $1.33 \mathrm{E}-12$ & $5.45 \mathrm{E}-12$ & $2.02 \mathrm{E}-12$ & $2.00 \mathrm{E} \quad 12$ \\
\hline 4.3 & $2.46 \mathrm{E}-12$ & $7.90 \mathrm{E}-13$ & $2.45 E-12$ & $9.01 \mathrm{E}-13$ & $1.19 \mathrm{E}-12$ & $4.86 \mathrm{E}-12$ & $1.80 \mathrm{E}-12$ & $1.78 \mathrm{E}-12$ \\
\hline 4.4 & $2.19 \mathrm{E}-12$ & $7.04 \mathrm{E}-13$ & $2.18 \mathrm{E}-12$ & $8.02 \mathrm{E}-13$ & $1.05 \mathrm{E} \quad 12$ & $4.32 \mathrm{E}-12$ & $1.60 \mathrm{E} \quad 12$ & $1.58 \mathrm{E}-12$ \\
\hline 4.5 & $1.95 \mathrm{E} \quad 12$ & $6.28 \mathrm{E}-13$ & $1.94 \mathrm{E} \quad 12$ & $7.14 \mathrm{E} \quad 13$ & $9.36 \mathrm{E}-13$ & $\begin{array}{lll}3.85 \mathrm{E} & 12\end{array}$ & $1.42 \mathrm{E} \quad 12$ & $1.40 \mathrm{E} \quad 12$ \\
\hline 4.6 & $1.74 \mathrm{E}-12$ & $5.60 E-13$ & $1.73 \mathrm{E}-12$ & 6.36E $\quad 13$ & $8.31 \mathrm{E}-13$ & $3.43 \mathrm{E}-12$ & $1.27 \mathrm{E}-12$ & $1.24 \mathrm{E}-12$ \\
\hline 4.7 & $\begin{array}{lll}1.55 \mathrm{E} & 12\end{array}$ & $4.99 \mathrm{E}-13$ & $1.54 \mathrm{E}-12$ & $\begin{array}{lll}5.6 G E & 13\end{array}$ & $7.36 \mathrm{E}-13$ & $\begin{array}{lll}3.05 \mathrm{E} & 12\end{array}$ & $1.13 \mathrm{E}-12$ & $1.10 \mathrm{E} \quad 12$ \\
\hline 4.8 & $1.38 \mathrm{E}-12$ & $4.45 \mathrm{E}-13$ & $1.37 \mathrm{E}-12$ & $\begin{array}{lll}5.03 E & 13\end{array}$ & $6.52 E-13$ & $2.71 \mathrm{E}-12$ & $1.00 \mathrm{E}-12$ & $9.75 E-13$ \\
\hline 4.9 & $1.23 \mathrm{E}-12$ & $3.97 \mathrm{E}-13$ & $1.21 \mathrm{E}-12$ & $4.47 \mathrm{E}-13$ & $5.76 \mathrm{E}-13$ & $2.41 \mathrm{E}-12$ & $8.92 \mathrm{E}-13$ & $8.61 \mathrm{E}-13$ \\
\hline 5.0 & 1.10 E 12 & $3.54 \mathrm{E}-13$ & $1.08 \mathrm{E}-12$ & $3.98 \mathrm{E}-13$ & $\begin{array}{lll}5.08 \mathrm{E} & 13\end{array}$ & $2.14 \mathrm{E}-12$ & $7.92 \mathrm{E}-13$ & $7.60 \mathrm{E}-13$ \\
\hline 5.1 & $9.80 E-13$ & $3.15 \mathrm{E}-13$ & $9.57 \mathrm{E}-13$ & $3.53 E-13$ & $4.47 \mathrm{E}-13$ & $1.90 \mathrm{E}-12$ & $7.03 E-13$ & $6.68 \mathrm{E}-13$ \\
\hline 5.2 & $8.74 E-13$ & $2.81 \mathrm{E}-13$ & $8.48 \mathrm{E}-13$ & $3.13 \mathrm{E}-13$ & $3.92 E-13$ & $1.68 \mathrm{E}-12$ & $6.24 \mathrm{E}-13$ & $5.86 \mathrm{E}-13$ \\
\hline 5.3 & $7.79 E-13$ & $2.51 \mathrm{E}-13$ & $7.51 \mathrm{E}-13$ & $2.77 \mathrm{E}-13$ & $3.42 \mathrm{E}-13$ & $1.49 \mathrm{E}-12$ & $5.53 \mathrm{E}-13$ & $5.12 \mathrm{E}-13$ \\
\hline 5.4 & $6.95 E-13$ & $2.24 \mathrm{E}-13$ & $6.64 \mathrm{E}-13$ & $2.45 \mathrm{E}-13$ & $2.98 \mathrm{E}-13$ & $1.32 \mathrm{E}-12$ & $4.89 \mathrm{E}-13$ & $4.46 \mathrm{E}-13$ \\
\hline 5.5 & $6.20 \mathrm{E}-13$ & $2.00 \mathrm{E}-13$ & $5.8 \mathrm{GE}-13$ & $2.17 \mathrm{E}-13$ & $2.58 \mathrm{E}-13$ & $1.16 \mathrm{E}-12$ & $4.31 \mathrm{E}-13$ & $3.86 \mathrm{E}-13$ \\
\hline 5.6 & $\begin{array}{lll}5.54 \mathrm{E} & 13\end{array}$ & $1.78 \mathrm{E}-13$ & $5.16 \mathrm{E}-13$ & $1.91 \mathrm{E}-13$ & $2.22 \mathrm{E} \quad 13$ & $1.02 \mathrm{E} \quad 12$ & $3.80 \mathrm{E}-13$ & $3.32 \mathrm{E}-13$ \\
\hline 5.7 & $4.94 \mathrm{E}-13$ & $1.59 \mathrm{E}-13$ & $4.53 \mathrm{E}-13$ & $1.68 \mathrm{E}-13$ & $1.90 \mathrm{E} \quad 13$ & $8.99 \mathrm{E}-13$ & $3.34 \mathrm{E}-13$ & $2.84 \mathrm{E} \quad 13$ \\
\hline 5.8 & $4.41 \mathrm{E}-13$ & $1.42 \mathrm{E}-13$ & $3.96 \mathrm{E}-13$ & $1.47 \mathrm{E}-13$ & $1.62 \mathrm{E}-13$ & $7.86 \mathrm{E}-13$ & $2.92 \mathrm{E}-13$ & $2.42 \mathrm{E}-13$ \\
\hline 5.9 & $3.94 \mathrm{E}-13$ & $1.27 \mathrm{E}-13$ & $3.46 \mathrm{E}-13$ & $1.28 \mathrm{E}-13$ & $1.36 \mathrm{E}-13$ & $6.85 E-13$ & $2.55 \mathrm{E}-13$ & $2.04 \mathrm{E}-13$ \\
\hline 6.0 & $3.51 \mathrm{E}-13$ & $1.13 \mathrm{E}-13$ & $3.00 \mathrm{E}-13$ & $1.11 \mathrm{E} \quad 13$ & $1.14 \mathrm{E} \quad 13$ & $5.95 E-13$ & $2.21 \mathrm{E}-13$ & $1.70 \mathrm{E}-13$ \\
\hline 6.1 & $3.13 E-13$ & $1.01 \mathrm{E}-13$ & $2.59 \mathrm{E}-13$ & $9.60 E-14$ & 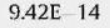 & $5.13 E-13$ & $1.91 \mathrm{E}-13$ & $1.41 \mathrm{E} \quad 13$ \\
\hline 6.2 & $2.80 \mathrm{E} \quad 13$ & $8.95 \mathrm{E} \quad 14$ & $2.22 \mathrm{E}-13$ & $8.24 \mathrm{E} \quad 14$ & $7.73 E-14$ & $4.41 \mathrm{E}-13$ & $1.64 \mathrm{E}-13$ & $1.16 \mathrm{E}-13$ \\
\hline 6.3 & $2.51 \mathrm{E} \quad 13$ & $7.97 \mathrm{E} \quad 14$ & $1.91 \mathrm{E}-13$ & $7.05 \mathrm{E}-14$ & $\begin{array}{lll}6.29 \mathrm{E} & 14\end{array}$ & $3.80 \mathrm{E}-13$ & $1.40 \mathrm{E}-13$ & $9.41 \mathrm{E}-14$ \\
\hline 6.4 & $2.28 \mathrm{E}-13$ & $7.18 \mathrm{E}-14$ & $1.66 \mathrm{E}-13$ & $6.05 E-14$ & $5.15 E-14$ & $3.34 \mathrm{E}-13$ & $1.21 \mathrm{E}-13$ & $7.71 \mathrm{E}-14$ \\
\hline 6.5 & $2.14 \mathrm{E}-13$ & $6.69 \mathrm{E}-14$ & $1.48 \mathrm{E}-13$ & $5.35 \mathrm{E}-14$ & $4.39 E-14$ & $3.11 \mathrm{E}-13$ & $1.08 \mathrm{E}-13$ & $6.60 \mathrm{E}-14$ \\
\hline 6.6 & $2.09 \mathrm{E}-13$ & 6.62E -14 & $1.40 \mathrm{E}-13$ & $\begin{array}{l}5.04 \mathrm{E} \quad 14 \\
\end{array}$ & $4.10 \mathrm{E} \quad 14$ & $3.12 \mathrm{E}-13$ & $1.05 \mathrm{E}-13$ & $6.23 \mathrm{E}-14$ \\
\hline 6.7 & $2.12 \mathrm{E}-13$ & $6.95 \mathrm{E}-14$ & $1.40 \mathrm{E}-13$ & $5.11 \mathrm{E}-14$ & $4.27 \mathrm{E}-14$ & $3.32 \mathrm{E}-13$ & $1.11 \mathrm{E}-13$ & $6.57 \mathrm{E}-14$ \\
\hline 6.8 & $2.18 \mathrm{E}-13$ & $7.51 \mathrm{E} \quad 14$ & $1.42 \mathrm{E}-13$ & $5.40 \mathrm{E} \quad 14$ & $4.72 E-14$ & $3.58 \mathrm{E}-13$ & $1.22 \mathrm{E}-13$ & $7.35 \mathrm{E}-14$ \\
\hline 6.9 & $2.19 \mathrm{E}-13$ & $8.00 E-14$ & $1.43 \mathrm{E}-13$ & $5.70 \mathrm{E}-14$ & $5.20 \mathrm{E}-14$ & $3.76 E-13$ & $1.33 \mathrm{E}-13$ & $8.15 E-14$ \\
\hline & & & & & & & (continue & ext page) \\
\hline
\end{tabular}


Table 3 (continued)

\begin{tabular}{|c|c|c|c|c|c|c|c|c|}
\hline \multirow[b]{2}{*}{ BE: } & \multicolumn{8}{|l|}{ Si XII } \\
\hline & -38.5 & -16.7 & -36.7 & -16.2 & -16.0 & -36.6 & -16.2 & -16.0 \\
\hline 7.0 & $2.14 \mathrm{E}-13$ & $8.18 \mathrm{E}-14$ & $1.39 \mathrm{E}-13$ & $5.80 \mathrm{E}-14$ & $5.46 \mathrm{E}-14$ & $3.76 \mathrm{E}-13$ & $1.38 \mathrm{E}-13$ & $8.61 \mathrm{E} \quad 14$ \\
\hline 7.1 & $2.01 \mathrm{E}-13$ & $7.96 \mathrm{E}-14$ & $1.29 \mathrm{E}-13$ & $5.62 \mathrm{E}-14$ & $5.42 \mathrm{E}-14$ & $3.58 \mathrm{E}-13$ & $1.36 \mathrm{E}-13$ & $8.57 \mathrm{E}-14$ \\
\hline 7.2 & $1.81 \mathrm{E}-13$ & $7.37 \mathrm{E}-14$ & $1.16 \mathrm{E}-13$ & $5.18 \mathrm{E}-14$ & $5.07 \mathrm{E}-14$ & $3.23 \mathrm{E}-13$ & $1.26 \mathrm{E}-13$ & $8.03 E-14$ \\
\hline 7.3 & $1.57 \mathrm{E}-13$ & $6.52 \mathrm{E}-14$ & $9.94 \mathrm{E}-14$ & $4.56 \mathrm{E}-14$ & $4.51 \mathrm{E}-14$ & $2.80 \mathrm{E}-13$ & $1.12 \mathrm{E}-13$ & $7.15 \mathrm{E}-14$ \\
\hline 7.4 & $1.32 \mathrm{E}-13$ & $5.55 E-14$ & $8.26 \mathrm{E}-14$ & $3.85 \mathrm{E}-14$ & $3.83 \mathrm{E}-14$ & $2.33 E-13$ & $9.46 \mathrm{E}-14$ & $6.09 \mathrm{E}-14$ \\
\hline 7.5 & $1.08 \mathrm{E}-13$ & $4.58 \mathrm{E}-14$ & $6.67 E-14$ & $3.15 \mathrm{E}-14$ & $3.15 \mathrm{E}-14$ & $1.89 \mathrm{E}-13$ & $7.76 \mathrm{E}-14$ & $5.00 \mathrm{E}-14$ \\
\hline 7.6 & $8.67 \mathrm{E}-14$ & $3.68 \mathrm{E}-14$ & $5.26 \mathrm{E}-14$ & $2.51 \mathrm{E}-14$ & $2.51 \mathrm{E}-14$ & $1.49 \mathrm{E}-13$ & $6.19 \mathrm{E}-14$ & $3.99 \mathrm{E}-14$ \\
\hline 7.7 & $6.84 \mathrm{E}-14$ & $2.90 \mathrm{E}-14$ & $4.07 \mathrm{E}-14$ & $1.96 \mathrm{E}-14$ & $1.95 \mathrm{E}-14$ & $1.15 \mathrm{E}-13$ & $4.82 \mathrm{E}-14$ & $3.10 \mathrm{E}-14$ \\
\hline 7.8 & $5.32 \mathrm{E}-14$ & $2.25 \mathrm{E}-14$ & $3.10 \mathrm{E}-14$ & $1.50 \mathrm{E}-14$ & $1.49 \mathrm{E}-14$ & $8.76 \mathrm{E}-14$ & $3.69 \mathrm{E}-14$ & $2.37 \mathrm{E}-14$ \\
\hline 7.9 & $4.09 \mathrm{E}-14$ & $1.72 E-14$ & $2.33 \mathrm{E}-14$ & $1.13 \mathrm{E}-14$ & $1.12 \mathrm{E}-14$ & $6.59 \mathrm{E}-14$ & $2.78 \mathrm{E}-14$ & $1.78 \mathrm{E}-14$ \\
\hline 8.0 & $3.12 \mathrm{E}-14$ & $1.30 \mathrm{E}-14$ & $1.74 \mathrm{E}-14$ & $8.43 E-15$ & $8.33 E-15$ & $4.90 E-14$ & $2.08 \mathrm{E}-14$ & $1.33 \mathrm{E}-14$ \\
\hline 8.1 & $2.36 \mathrm{E}-14$ & $9.73 E-15$ & $1.29 \mathrm{E}-14$ & $6.23 E-15$ & $6.13 E-15$ & $3.61 \mathrm{E}-14$ & $1.53 \mathrm{E}-14$ & $9.76 \mathrm{E}-15$ \\
\hline 8.2 & $1.77 \mathrm{E}-14$ & $7.24 \mathrm{E}-15$ & $9.46 E-15$ & $4.58 \mathrm{E}-15$ & $4.48 \mathrm{E}-15$ & $2.65 E-14$ & $1.12 \mathrm{E} \quad 14$ & $7.13 \mathrm{E}-15$ \\
\hline 8.3 & $1.33 \mathrm{E}-14$ & $5.36 \mathrm{E}-15$ & $6.92 \mathrm{E}-15$ & $3.34 \mathrm{E}-15$ & $3.25 \mathrm{E}-15$ & $1.93 \mathrm{E}-14$ & $8.20 \mathrm{E}-15$ & $5.17 \mathrm{E}-15$ \\
\hline 8.4 & $9.87 \mathrm{E}-15$ & $3.96 \mathrm{E}-15$ & $5.04 \mathrm{E}-15$ & $2.43 E-15$ & $2.35 \mathrm{E}-15$ & $1.40 \mathrm{E}-14$ & $5.95 E-15$ & $3.74 \mathrm{E}-15$ \\
\hline 8.5 & $7.33 E-15$ & $2.91 \mathrm{E}-15$ & $3.66 \mathrm{E}-15$ & $1.76 \mathrm{E}-15$ & $1.69 \mathrm{E}-15$ & $1.02 \mathrm{E}-14$ & $4.30 \mathrm{E}-15$ & $2.69 E-15$ \\
\hline 8.6 & $5.43 \mathrm{E}-15$ & $2.13 E-15$ & $2.66 \mathrm{E}-15$ & $1.27 \mathrm{E}-15$ & $1.21 \mathrm{E}-15$ & $7.33 \mathrm{E}-15$ & $3.10 \mathrm{E}-15$ & $1.93 \mathrm{E}-15$ \\
\hline 8.7 & $4.01 \mathrm{E}-15$ & $1.56 \mathrm{E}-15$ & $1.92 \mathrm{E}-15$ & $9.15 E-16$ & $8.66 \mathrm{E}-16$ & $5.28 \mathrm{E}-15$ & $2.23 E-15$ & $1.38 \mathrm{E}-15$ \\
\hline 8.8 & $2.96 \mathrm{E}-15$ & $1.14 \mathrm{E}-15$ & $1.39 \mathrm{E}-15$ & $6.58 \mathrm{E}-16$ & $6.18 \mathrm{E}-16$ & $3.80 \mathrm{E}-15$ & $1.60 \mathrm{E}-15$ & $9.85 \mathrm{E}-16$ \\
\hline 8.9 & $2.18 \mathrm{E}-15$ & $8.28 \mathrm{E}-16$ & $1.00 \mathrm{E}-15$ & $4.73 E-16$ & $4.41 \mathrm{E}-16$ & $2.73 E-15$ & $1.15 \mathrm{E}-15$ & $7.02 \mathrm{E}-16$ \\
\hline 9.0 & $1.60 \mathrm{E}-15$ & $6.02 E-16$ & $7.20 \mathrm{E} \quad 16$ & $3.39 \mathrm{E}-16$ & $3.14 \mathrm{E}-16$ & $1.96 \mathrm{E}-15$ & $8.24 \mathrm{E}-16$ & $5.00 \mathrm{E}-16$ \\
\hline
\end{tabular}

BE is the binding energy of the level in Rydberg. 


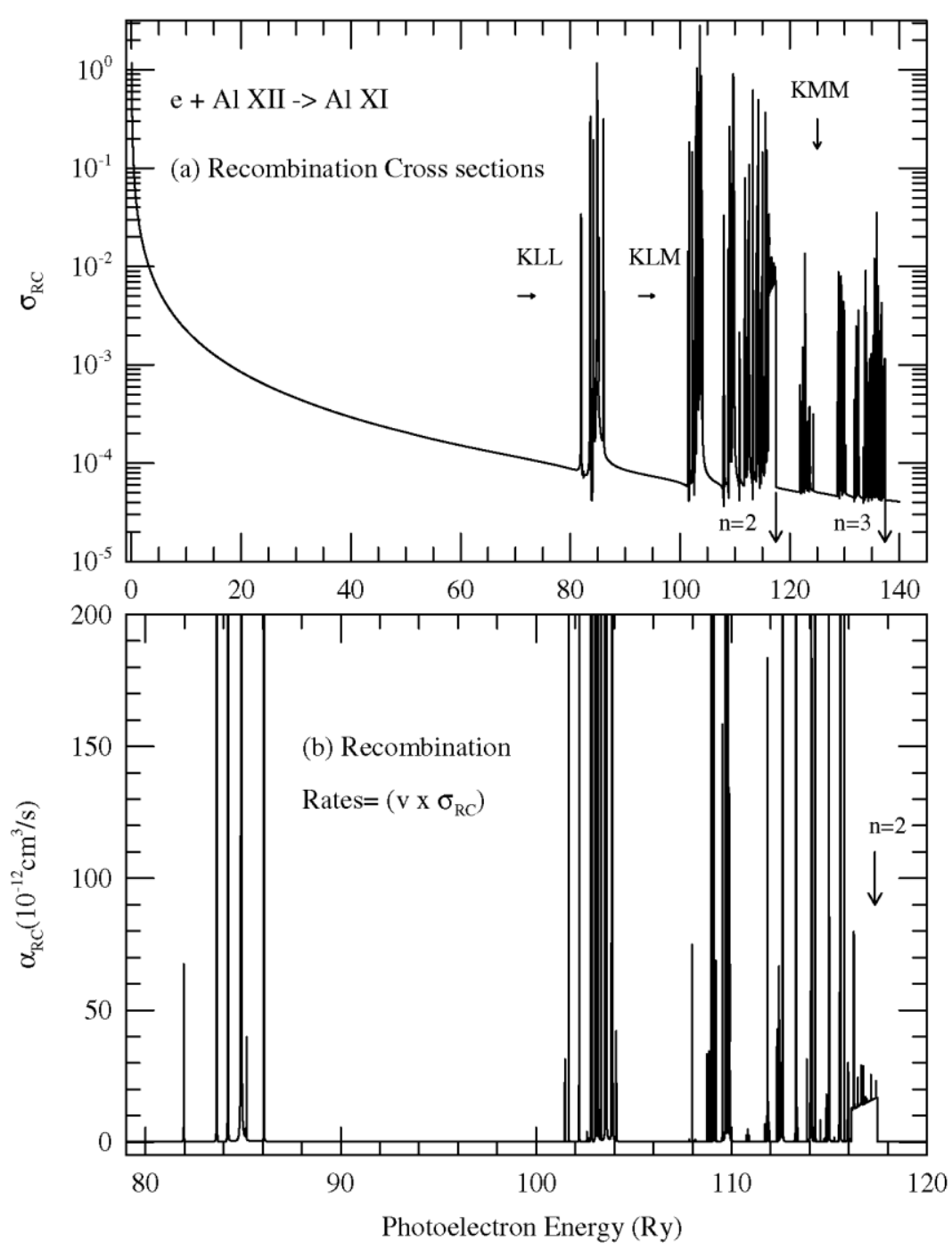

Fig. 4. (a) Total unified (e + ion) recombination cross-sections, $\sigma_{\mathrm{RC}}$, and (b) unified recombination rate coefficients, $\alpha_{\mathrm{RC}}(\mathrm{E})$, versus photoelectron energy for $(\mathrm{e}+\mathrm{Al} \mathrm{XII} \rightarrow \mathrm{Al}$ XI). Separated resonance complexes, KLL, KLM, etc., of $\mathrm{n}$ $=2$ and $\mathrm{KMM}$, etc., of $\mathrm{n}=3$ thresholds are noted in (a). $\alpha_{\mathrm{RC}}(\mathrm{E})$ can be measured experimentally.

Radiation damping of resonances up to $\mathrm{n}=10$ are included using the extended codes STGF and STGBF (Nahar and Pradhan, 1994; Zhang et al., 1999). The narrow resonances of these ions were delineated at a very fine energy mesh. The program PBPRAD extends the total photoionization cross-sections in the high energy region beyond the highest threshold in target ion by a 'tail' using a fitting formula, $\sigma_{\mathrm{PI}}^{\mathrm{o}}\left(\mathrm{E}^{\mathrm{o}} / \mathrm{E}\right)^{\mathrm{m}}$, where $\mathrm{E}^{\mathrm{o}}$ is the last tabulated energy beyond the resonances, that is, at an energy beyond the highest target threshold and $-1 \geq \mathrm{m} \geq-3$, as described in Nahar and Pradhan (1994). $m=-3$ for Kramers fit at very high energies. The DR collision strengths for the narrow and dense resonances in the energy region, $v_{\mathrm{o}}<v \leq \infty$, below each target excited threshold were obtained using STGFDR. The radiative decay rates for the allowed excited thresholds used for $\Omega(\mathrm{DR})$ are given in Table 2 .

Level-specific recombination cross-sections, $\sigma_{\mathrm{RC}}(\mathrm{i})$, into various bound levels $i=n(S L J)$ of the recombined $\left(\mathrm{e}+\right.$ ion) system, are obtained from partial photoionization cross-sections $\sigma_{\mathrm{PI}}(\mathrm{i}, \mathrm{g})$ of the level $\mathrm{i}$ using the program RECXS. It sums up the level specific rates and adds with the 
resonant contributions of high-n $\left(v_{\mathrm{o}} \leq v \leq \infty\right) \mathrm{DR}$ for the total recombination rates. As an additional check on the numerical calculations, the total recombination rate coeffi-

cients, $\alpha_{R}$, are also calculated from the total recombination collision strength, $\Omega_{R C}$, obtained from all the photoionization cross-sections, and the DR collision strengths. The difference between the two numerical values is a few percent.

\section{Results and discussions}

Self-consistent set of results are presented for photoionization and electron-ion recombination of $(\mathrm{Al} \mathrm{XI}+h v \rightleftharpoons \mathrm{Al} \mathrm{XII}+\mathrm{e}),(\mathrm{Al} \mathrm{XII}+h v \rightleftharpoons \mathrm{Al} \mathrm{XIII}+\mathrm{e}),(\mathrm{Si} \mathrm{XII}+h v \rightleftharpoons \mathrm{Si} \mathrm{XIII}+$ e) and (Si XIII $+h v \rightleftharpoons \mathrm{Si} \mathrm{XIV}+\mathrm{e}$ ). The set includes total and partial photoionization cross-sections and recombination rate coefficients for all fine structure levels up to $n \leq 10$.

Main features of photoionization and electron-ion recombination of Li-like Al XI and Si XII and He-like Al XII and Si XIII are presented in separate sections below.

\subsection{Al XI and Si XII}

A total of 98 bound levels are obtained for Li-like Al XI and Si XII with $n \leq 10,0 \leq 1 \leq 9,0$ $\leq \mathrm{L} \leq 11$, and total angular momentum $1 /$

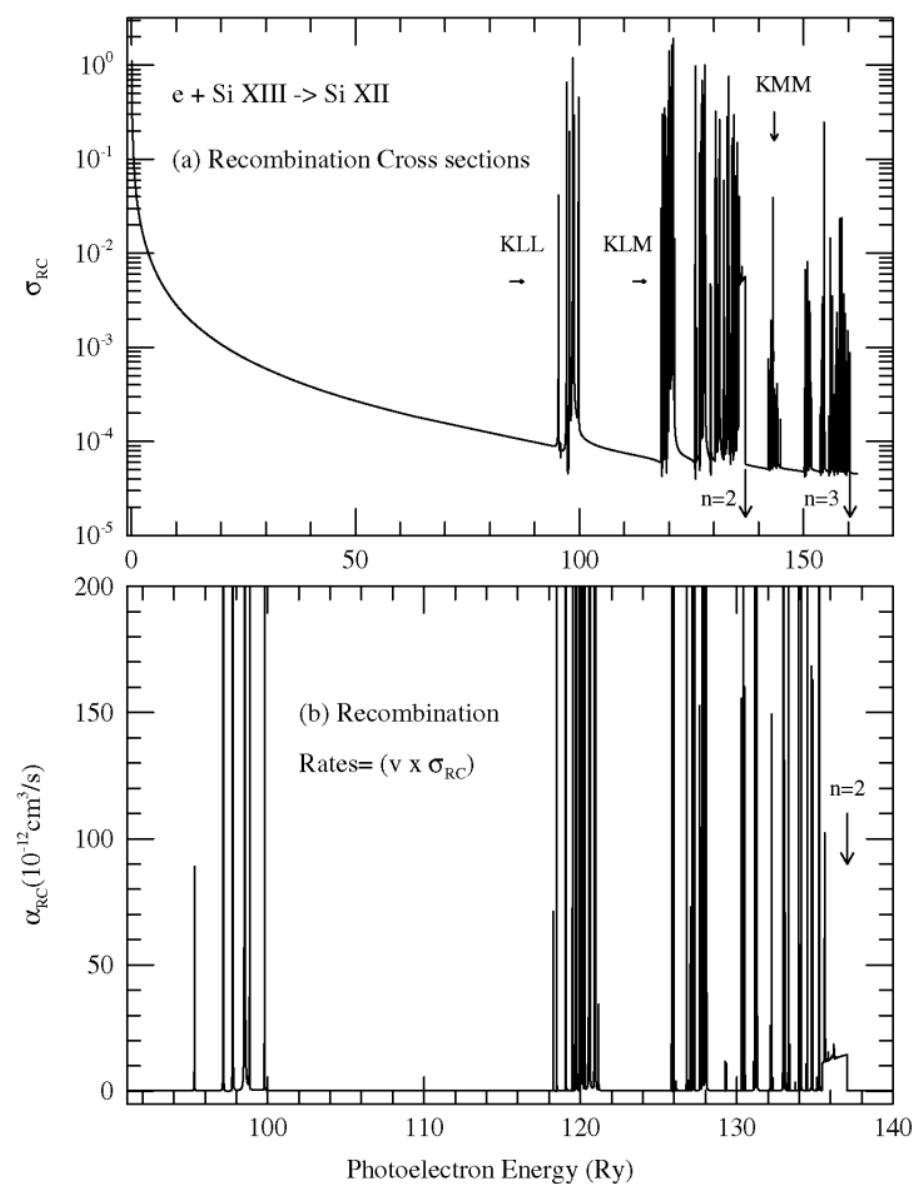

Fig. 5. (a) Total unified $\left(e+\right.$ ion) recombination cross-sections, $\sigma_{R C}$, and (b) unified recombination rate coefficients, 
$\mathrm{a}_{\mathrm{RC}}(\mathrm{E})$, versus photoelectron energy for $(\mathrm{e}+\mathrm{Si}$ XIII $\rightarrow$ Si XII). Separated resonant complexes, KLL, KLM, etc., of $\mathrm{n}=$ 2 and $\mathrm{KMM}, \mathrm{KMN}$, etc., of $\mathrm{n}=3$ thresholds are noted in (a). $\alpha_{\mathrm{RC}}(\mathrm{E})$ can be measured experimentally.

$2 \leq J \leq 17 / 2$ (Nahar, 2002). The earlier results (Nahar, 2002) are for oscillator strengths for radiative transitions in the BPRM approximation and hence are consistent with the present results for photoionization and electron-ion recombination as they are obtained using the same wavefunction expansion.

\subsubsection{Photoionization}

The total photoionization cross-section $\left(\sigma_{\mathrm{PI}}\right)$, which include contributions from all channels for ionization in to ground and various excited states of the residual ion, are presented for all 98 fine structure levels of Al XI and Si XII. Partial photoionization refers to ionization in to the ground state of the core ion. These are also presented for all 98 fine structure bound levels. Important characteristics in $\sigma_{\mathrm{PI}}$ are illustrated below.

Photoionization cross-section of the ground level are needed for various models. $\sigma_{\mathrm{PI}}$ for the ground $1 \mathrm{~s}^{2} 2 \mathrm{~s}^{2} \mathrm{~S}_{1 / 2}$ level of Al XI and Si XII shown in Figs. 1 and 2, respectively. They show typical features of a few electron systems: decaying smoothly over a wide energy range before appearance of resonances belonging to high lying core states. In Figs. 1 and 2, the top panel (a) presents the total photoionization cross-section and the bottom panel (b) presents the partial cross-sections for ionization to residual ions $\mathrm{Al}$ XII and Si XIII. The resonances in $\sigma_{\mathrm{PI}}$ are due to various Rydberg series $1 \mathrm{~s} n l v l^{\prime}$ converging on to the $\mathrm{n}=2,3$ levels of the core ion. $v$ is the effective quantum number for the autoionizing state. The first resonance complexes are the well known KLL, KLM, etc., complexes belonging to $\mathrm{n}=2$ core thresholds. KLL denotes the series $1 \mathrm{~s} 2121$, KLM denotes 1s2131, etc. The total and partial cross-sections are identical below the first excited level of the residual ion. However, the total $\sigma_{P I}$ is enhanced beyond $n=2$ thresholds by the added contributions from excited channels in contrast to the partial cross-sections where they are not added. The enhancement at the excited $n=2$ thresholds in Fig. 1 is due to the K-shell photoionization:

$$
h v+\left(1 \mathrm{~s}^{2} 2 \mathrm{~s}\right) \rightarrow \mathrm{e}+(1 \mathrm{~s} 2 \mathrm{~s}, 1 \mathrm{~s} 2 \mathrm{p})
$$

which is at about $150 \mathrm{Ry}$ (sum of ground level energy $32.485 \mathrm{Ry}$ and core excitation, $1 \mathrm{~s}^{21} \mathrm{~S}_{0}$ $1 \mathrm{~s} 2 \mathrm{p}^{1} \mathrm{P}_{1}^{\mathrm{o}}$, energy $117.4 \mathrm{Ry}$ ) for $\mathrm{Al} \mathrm{XI}$ and $175.5 \mathrm{Ry}$ (sum of ground level energy $38.47 \mathrm{Ry}$ and core excitation, $1 \mathrm{~s}^{21} \mathrm{~S}_{0}-1 \mathrm{~s} 2 \mathrm{p}^{1} \mathrm{P}^{\mathrm{o}}{ }_{1}$, energy $137.07 \mathrm{Ry}$ ) for Si XII. This inner-shell edge plays an important role in $\mathrm{X}$-ray photoionization models.

Fig. 3 presents total $\sigma_{\mathrm{PI}}$ of excited Rydberg series of levels $1 \mathrm{~s}^{2} \mathrm{np}\left({ }^{2} \mathrm{P}_{1 / 2}^{\mathrm{o}}\right), 2 \leq n \leq 7$ of $\mathrm{Al} \mathrm{XI}$ illustrating the resonant structures 


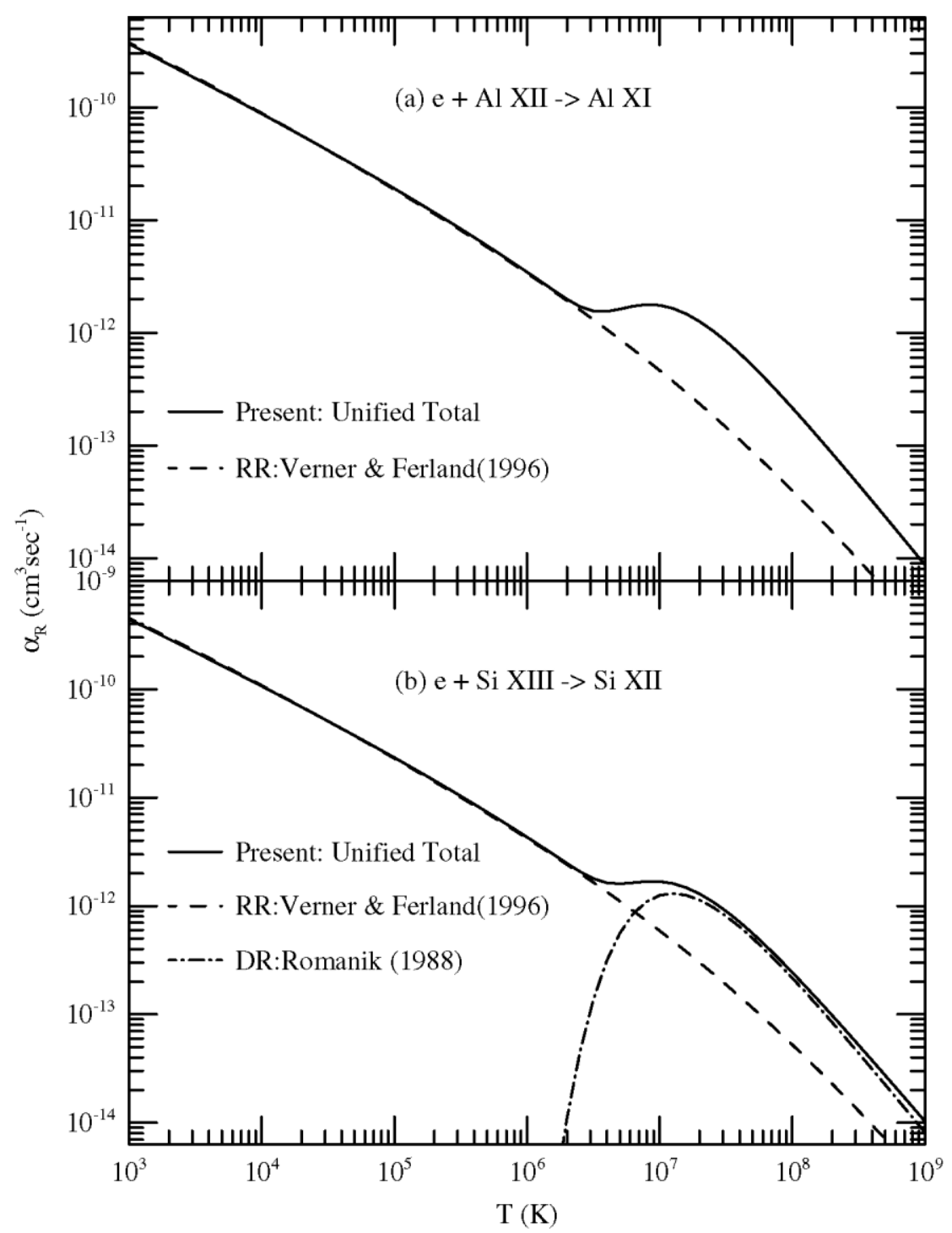

Fig. 6. Total unified recombination rate coefficients $\alpha_{R}(T)$ (solid curves) for (a) Al XI and (b) Si XII. The RR rates are by Verner and Ferland (1996, dash) and the DR rates are by Romanik (1988, dot-dash).

at higher energies, especially the photoexcitation-of-core (PEC) resonances. Contrary to hydrogenic decay of the excited state cross-sections, PEC resonances enhance the background. These resonances are manifested by dipole transitions in the core ion and appear at the excited thresholds of transitions. At photon energies that equal to core excitation energies, the core goes through the allowed $\Delta J=0-1$ transitions while the outer electron remains a 'spectator' in a doubly-excited resonant state. The state decays via autoionization in to the ground level of the core. PEC resonances are pointed by arrows in Fig. 3, at about 117 Ry for $\mathrm{n}=2$ thresholds $1 \mathrm{~s} 2 \mathrm{p}$ $\mathrm{P}_{1}^{\mathrm{o}}{ }_{1}, \mathrm{P}^{\mathrm{o}}{ }_{1}$ and at about $137 \mathrm{Ry}$ of $\mathrm{n}=3$ threshoulds $1 \mathrm{~s} 3 \mathrm{p}^{3} \mathrm{P}_{1}^{\mathrm{o}},{ }^{1} \mathrm{P}_{1}^{\mathrm{o}}$. These resonances are more enhanced than the narrow Rydberg resonances and can increase the background cross-sections by orders of magnitude. The effect becomes increasingly prominent in cross-sections of higher excited levels. PEC resonances exist in $\sigma_{\mathrm{PI}}$ of all excited bound levels of Al XI and Si XII.

The partial cross-sections also show PEC resonances due to core excitation. However, the partial cross-sections have lower background cross-sections and less resonances. 


\subsubsection{Electron-ion recombination}

Level-specific recombination rate coefficients $\alpha_{R}(T, n S L J)$ are obtained for all 98 fine structure levels of $\mathrm{Al} \mathrm{XI}$ and Si XII. Table 3 presents $\alpha_{R}(\mathrm{~T}, \mathrm{nSLJ})$ for recombination in to the ground and lowest seven excited $\mathrm{n}=2$ and 3 levels of Al XI and Si XII: $1 \mathrm{~s}^{2} 2 \mathrm{~s}^{2} \mathrm{~S}_{0}, 1 \mathrm{~s}^{2} 2 \mathrm{p}^{2} \mathrm{P}_{1 / 2,3 / 2}^{0}$, $1 \mathrm{~s}^{2} 3 \mathrm{~s}^{2} \mathrm{~S}_{0}, 1 \mathrm{~s}^{2} 3 \mathrm{p}^{2} \mathrm{P}_{1 / 2,3 / 2}^{\mathrm{o}}$, and $1 \mathrm{~s}^{2} 3 \mathrm{~d}^{2} \mathrm{D}_{3 / 2,5 / 2}$. Spectral modeling in the $\mathrm{UV}$ region often requires these rates. Typical feature of these rates show smooth decay with a DR 'shoulder' at high temperature.

The total recombination cross-sections $\sigma_{\mathrm{RC}}(\mathrm{E})$ and the recombination collision strengths $\sigma_{\mathrm{RC}}(\mathrm{E})$ are related to each other through kinematic factors and hence show similar detailed resonant features. Hence only $\sigma_{\mathrm{RC}}(\mathrm{E})$ is discussed. The unified total $\sigma_{\mathrm{RC}}$ for $\mathrm{Al}$ XI and Si XII are presented in the top panel of Figs. 4 and 5, respectively. It diverges at zero photoelectron energy, but decays fast smoothly with energy until the emergence of resonance complexes at high energies. A few resonance complexes are marked in the figures - KLL, KLM, KLN, etc., converging onto the $n=2$ thresholds, and KMM, KMN, etc., converging onto the $n=3$ thresholds.

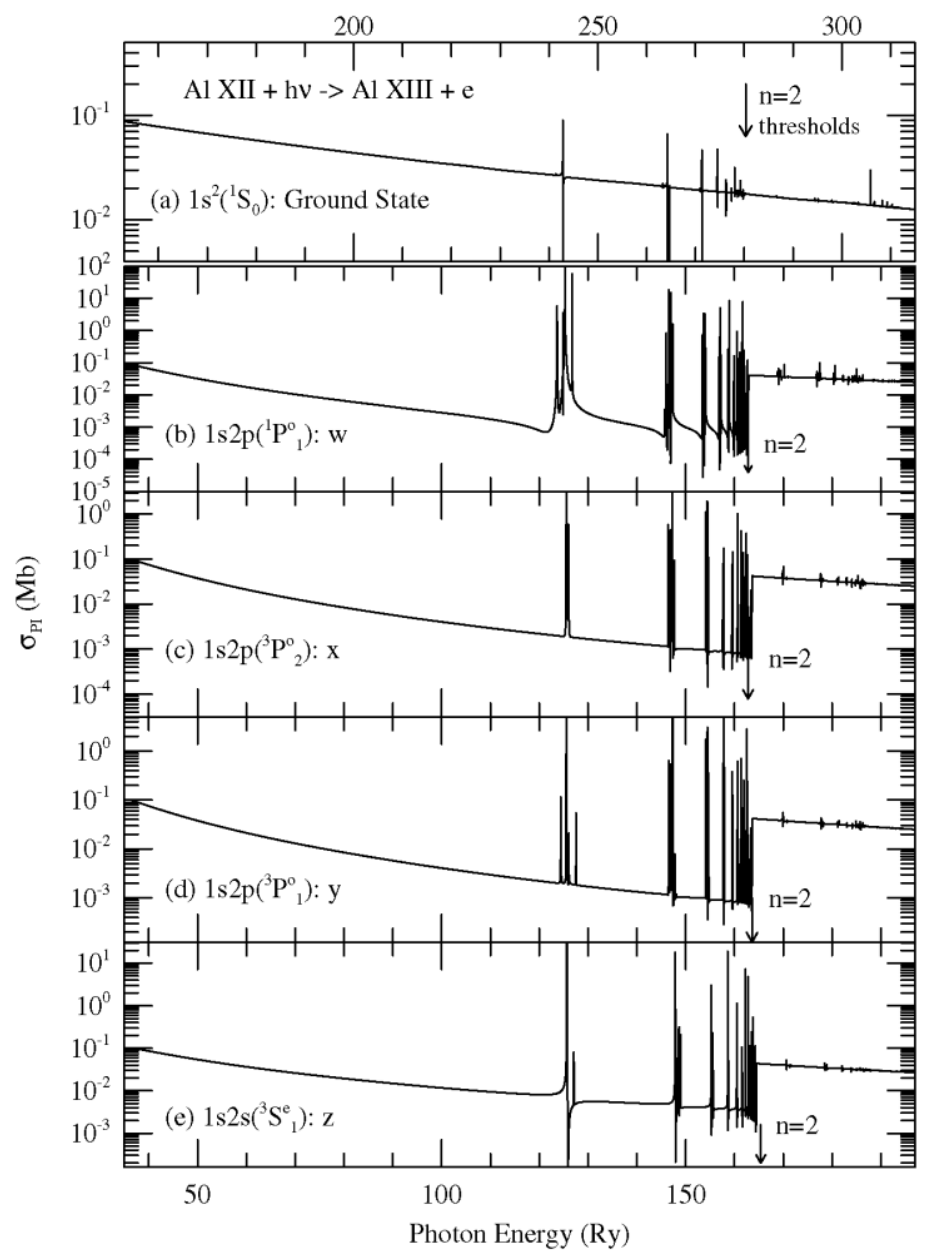

Fig. 7. Level-specific photoionization cross-sections of $\mathrm{Al} \mathrm{XII:} \mathrm{(a)} \mathrm{the} \mathrm{ground} \mathrm{level,} 1 \mathrm{~s}^{2}\left({ }^{1} \mathrm{~S}_{0}\right)$, and four excited $(b)$ $1 \mathrm{~s} 2 \mathrm{p}\left({ }^{1} \mathrm{P}_{1}^{\mathrm{o}}\right),(c) 1 \mathrm{~s} 2 \mathrm{p}\left({ }^{3} \mathrm{P}_{2}^{\mathrm{o}}\right),(d) 1 \mathrm{~s} 2 \mathrm{p}\left({ }^{3} \mathrm{P}_{1}^{\mathrm{o}}\right),(e) 1 \mathrm{~s} 2 \mathrm{~s}\left({ }^{3} \mathrm{~S}_{1}\right)$ levels corresponding to the prominent resonance $(w)$, intercombination $(y)$, and forbidden $(x, z)$ diagnostic X-ray lines. K-shell ionization edge is noticed at the $\mathrm{n}=2$ thresholds for the excited levels. 
These resonances, especially the KLL resonances manifest themselves as dielectronic satellite lines observed in tokamaks, Electron-Beam-Ion-Traps (EBIT), ion storage rings, and high temperature astrophysical sources. The profiles and intensities from the unified cross-sections can be directly compared with measured quantities and observed recombination spectra of satellite lines (Nahar and Pradhan, 2006).

The photorecombination rates, $\alpha_{\mathrm{RC}}(\mathrm{E})$ where $\mathrm{E}$ is the photoelectron energy, for $\mathrm{Al} \mathrm{XI}$ and Si XII are presented in the lower panels of Figs. 4 and 5, respectively. These are of interest for comparison with experiments and hence are presented in the energy range where the resonant part of $\alpha_{\mathrm{RC}}(\mathrm{E})$ converge on to the $\mathrm{n}=2$ thresholds of the respective cores. They show high-peak narrow resonances. In experiments these resonances appear in overlapped broad resonances due to convolution by the monochromatic bandwidth of the detector.

The total recombination rate coefficient $\alpha_{\mathrm{RC}}(\mathrm{T})$ for $\mathrm{Al}$ XI and Si XII are given for a wide range of temperature in Table 5 and the main features are illustrated and compared with the available data in Fig. 6a and b. These three-electron atomic systems show the basic features of total unified recombination rate coefficient (solid curves): starting high in the very low temperature, the rate decays until the rise due to DR at high temperature and then follows a monotonic decay. Low temperature recombination is dominated by radiative recombination (RR) into an infinite number of high-n levels while the rise at high temperature is due to appearance of resonances and the consequent dominance of DR.

The low-T unified recombination rate coefficients for Al XI and Si XII agree very well with the RR rate coefficients by Verner and Ferland (1996, dash). The agreement of high temperature DR rate for Si XII by Romanik (1988, dot-dash) who employed isolated resonance approximation is also good with the total unified rate. Good agreement between unified rates with the individual RR and DR rates are expected as the interference between RR and DR is negligible for these highly charged few electron systems.

\subsection{Al XII and Si XIII}

He-like Al XII and Si XIII have more bound levels than the Li-like ions. A total of 190 fine structure levels for Al XII and 189 for Si XIII 


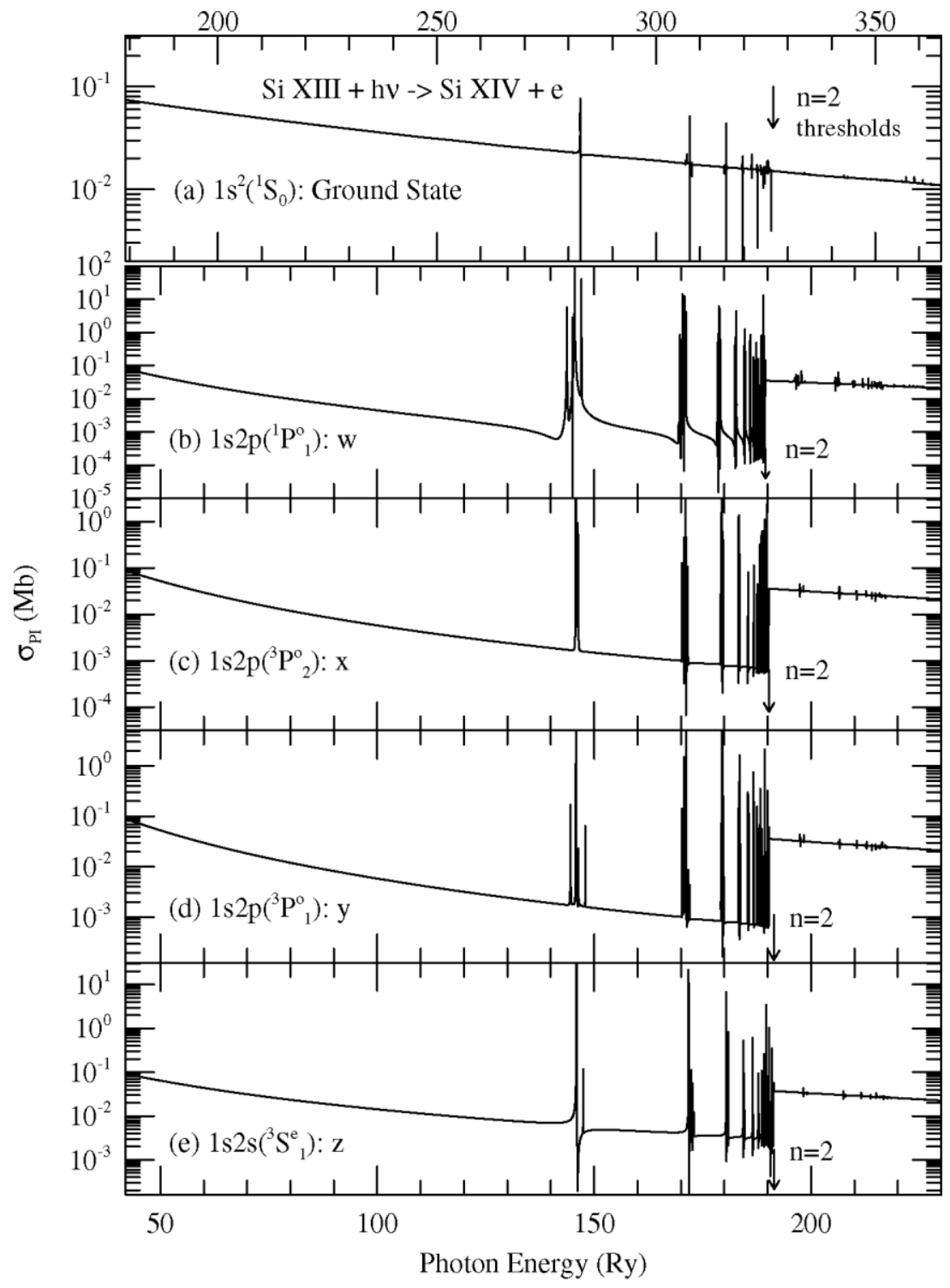

Fig. 8. Level-specific photoionization cross-sections of Si XIII: (a) the ground level, $1 \mathrm{~s}^{2}\left({ }^{1} \mathrm{~S}_{0}\right.$ ), and four excited (b) $1 \mathrm{~s} 2 \mathrm{p}\left({ }^{1} \mathrm{P}_{1}^{\mathrm{o}}\right)$, (c) $1 \mathrm{~s} 2 \mathrm{p}\left({ }^{3} \mathrm{P}_{2}^{\mathrm{o}}\right)$, (d) $1 \mathrm{~s} 2 \mathrm{p}\left({ }^{3} \mathrm{P}_{1}^{\mathrm{o}}\right)$, (e) $1 \mathrm{~s} 2 \mathrm{~s}\left({ }^{3} \mathrm{~S}_{1}\right)$ levels corresponding to the prominent resonance (w), intercombination $(\mathrm{y})$, and forbidden $(\mathrm{x}, \mathrm{z})$ diagnostic X-ray lines. K-shell ionization edge is noticed at the $\mathrm{n}=2$ thresholds for the excited levels.

with $n \leq 10,0 \leq l \leq 9,0 \leq L \leq 14$, and total angular momentum $0 \leq J \leq 10$ are found. The energies agree very well, in less than $1 \%$, with the available observed energies compiled by NIST.

The following two subsections describe the important features for photoionization and recombination for these ions.

\subsubsection{Photoionization}

Total photoionization cross-sections for Al XI and Si XII, leaving the target or core ion in the ground and 15 excited levels, are presented for all bound levels with $\mathrm{n} \leq 10$. Partial photoionization cross-sections leaving the target in the ground level are also obtained for all fine structure levels of these ions. 
Illustrative features of the total $\sigma_{\mathrm{PI}_{\mathrm{I}}}$ of the ground and low lying excited levels are shown in Fig. 7 for Al XII and Fig. 8 for Si XIII, respectively. In each figure, the top panel presents $\sigma_{\mathrm{PI}}$ of (a) the ground level $1 \mathrm{~s}^{2}\left({ }^{1} \mathrm{~S}_{0}\right)$ and the lower four panels (b-e) present level-specific cross-sections of the four lowest $\mathrm{n}=2$ excited levels corresponding to the prominent X-ray lines in the Ka complex: (b) resonant line (w: $\left.1 \mathrm{~s}^{2}\left({ }^{1} \mathrm{~S}_{0}\right) \leftarrow 1 \mathrm{~s} 2 \mathrm{p}\left({ }^{1} \mathrm{P}_{1}^{\mathrm{o}}\right)\right)$, (c) forbidden line $\left(\mathrm{x}: 1 \mathrm{~s}^{2}\left({ }^{1} \mathrm{~S}_{0}\right) \leftarrow 1 \mathrm{~s} 2 \mathrm{p}\left({ }^{3} \mathrm{P}{ }^{0}{ }_{2}\right)\right)$, (d) intercombination line (y: $\left.1 \mathrm{~s}^{2}\left({ }^{1} \mathrm{~S}_{0}\right) \leftarrow 1 \mathrm{~s} 2 \mathrm{p}\left({ }^{3} \mathrm{P}_{1}^{\mathrm{o}}\right)\right)$, and (e) forbidden line $\left(\mathrm{z}: 1 \mathrm{~s}^{2}\left({ }^{1} \mathrm{~S}_{0}\right) \leftarrow 1 \mathrm{~s} 2 \mathrm{~s}\left({ }^{3} \mathrm{~S} 1\right)\right)$, respectively. These four lines are the most commonly used spectral diagnostics of temperature, density, ionization balance, and abundances in plasma sources.

The figures show that the cross-section for both Al XII and Si XIII decrease monotonically over a large energy range for all levels. As the excited core levels of these ions are in high energy, the resonances in photoionization cross-sections do not appear for a relatively large energy range. $\sigma_{\mathrm{PI}}$ of the ground level has smooth background with some narrow resonances belonging to $n=2$ thresholds. However, more resonances are formed in excited state $\sigma_{\mathrm{PI}}$. In Figs. 7 and 8 the well-separated resonance complexes are the LL (2121), LM (2131'), etc., resonances converging on to $\mathrm{n}=2$ thresholds of the core and are more distinct than those of the ground level. In contrast to the ground level, the excited levels have a K-shell jump at the $n=2$ threshold due to $1 s-2 p$ transition:

$h v+(1 \mathrm{~s} 2 \mathrm{~s}, 1 \mathrm{~s} 2 \mathrm{p}) \rightarrow \mathrm{e}+(2 \mathrm{~s}, 2 \mathrm{p})$.

The ground level does not have an excited core electron to introduce such a jump. 


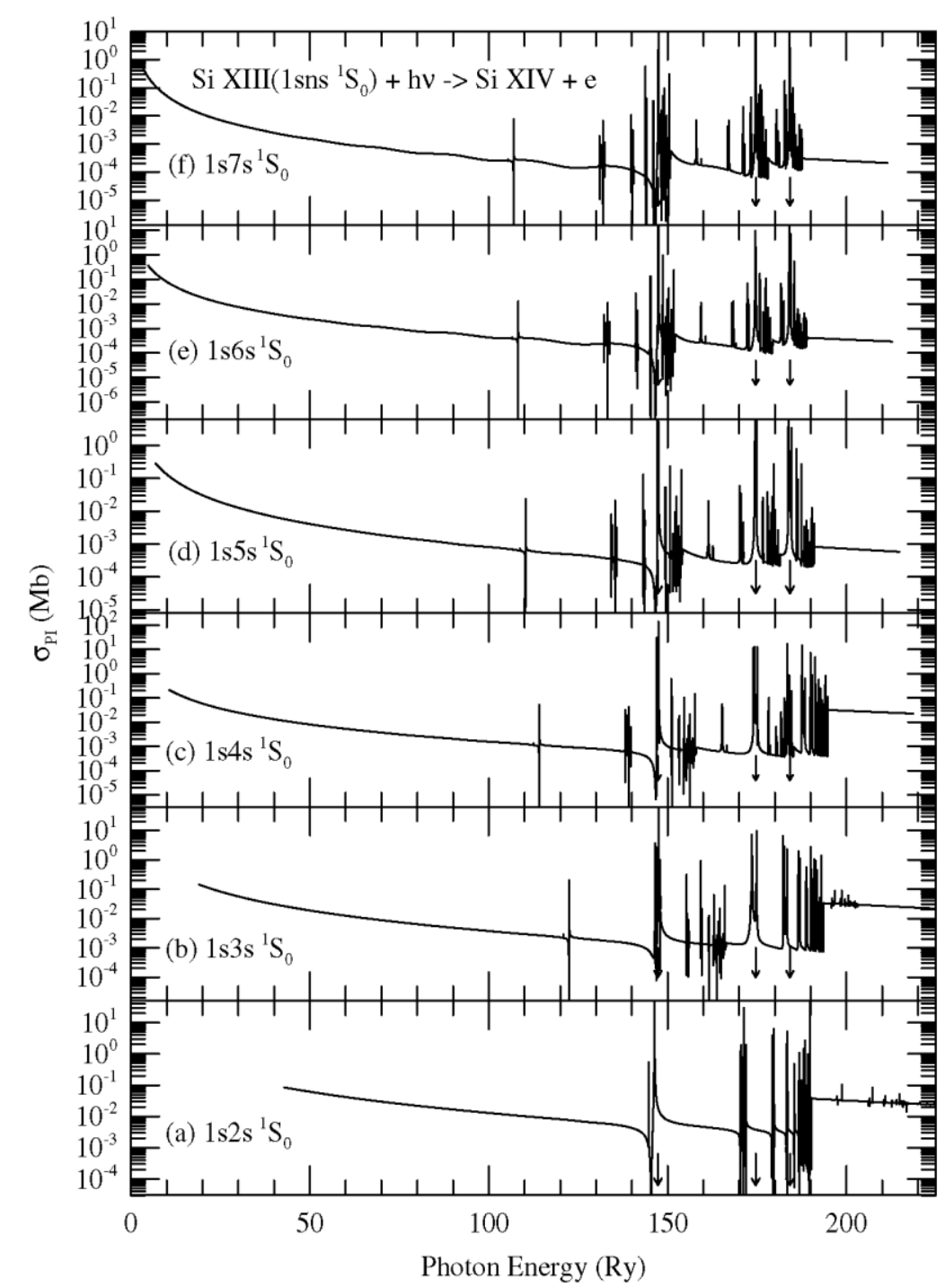

Fig. 9. Photoionization cross-sections of the Rydberg series of levels, $1 \mathrm{sns}\left({ }^{1} \mathrm{~S}_{0}\right)$ with $26 \mathrm{n} 67$, of Si XIII. Positions of prominent PEC (photoexcitation-of-core) resonances are shown by arrows at about 147 Ry for core excitations of $n=$ 2 levels $2 p^{2} \mathrm{P}_{1 / 2,3 / 2}^{\mathrm{o}}$, at about 175 Ry for $\mathrm{n}=3$ core levels $3 \mathrm{p}^{2} \mathrm{P}_{1 / 2,3 / 2}^{\mathrm{o}}$, and at about 184 Ry for $\mathrm{n}=4$ core levels $4 \mathrm{p}^{2} \mathrm{P}_{1 / 2,3 / 2}^{\mathrm{o}}$ 
Table 4

Level specific recombination rate coefficients for the ground and excited $2 l$ and $3 \mathrm{~s}$ levels of Al XII and Si XIII

\begin{tabular}{|c|c|c|c|c|c|c|c|c|c|}
\hline \multirow[t]{2}{*}{$\log _{10} T(\mathrm{~K})$} & \multicolumn{9}{|c|}{$\alpha_{\mathrm{R}}\left(\mathrm{cm}^{3} \mathrm{~s}^{-1}\right)$} \\
\hline & $1 \mathrm{~s}^{21} \mathrm{~S}$ & $1 \mathrm{~s} 2 \mathrm{~s}^{1} \mathrm{~S}_{0}$ & $1 \mathrm{~s}^{2} 3 \mathrm{~s}^{1} \mathrm{~S}_{0}$ & $1 \mathrm{~s} 2 \mathrm{p}^{3} \mathrm{P}_{0}^{0}$ & $1 \mathrm{~s} 2 \mathrm{~s}^{3} \mathrm{~s}_{1}$ & $1 \mathrm{~s} 3 \mathrm{~s}^{3} \mathrm{~S}_{1}$ & $1 \mathrm{~s} 2 \mathrm{p}^{3} \mathrm{P}_{1}^{0}$ & $1 \mathrm{~s} 2 \mathrm{p}^{1} \mathrm{P}_{1}^{0}$ & $1 \mathrm{~s} 2 \mathrm{p}^{3} \mathrm{P}_{2}^{0}$ \\
\hline \multirow[b]{2}{*}{ BE: } & \multicolumn{9}{|l|}{ Al XII } \\
\hline & 153.0 & 36.5 & -16.2 & 36.6 & 37.6 & -16.4 & -36.6 & 35.8 & 36.5 \\
\hline 1.0 & $4.23 \mathrm{E}-10$ & $2.74 \mathrm{E}-11$ & $9.09 \mathrm{E}-12$ & $2.71 \mathrm{E}-11$ & $8.52 \mathrm{E}-11$ & $2.76 \mathrm{E}-11$ & $8.14 \mathrm{E}-11$ & $7.31 \mathrm{E}-11$ & $1.35 \mathrm{E}-10$ \\
\hline 1.1 & $3.77 \mathrm{E}-10$ & $2.44 \mathrm{E}-11$ & $8.10 \mathrm{E}-12$ & $2.42 \mathrm{E}-11$ & $7.59 \mathrm{E}-11$ & $2.46 \mathrm{E}-11$ & $7.25 \mathrm{E}-11$ & $6.51 \mathrm{E}-11$ & $1.20 \mathrm{E}-10$ \\
\hline 1.2 & $3.36 \mathrm{E}-10$ & $2.18 \mathrm{E}-11$ & $7.22 \mathrm{E}-12$ & $2.15 \mathrm{E}-11$ & $6.77 \mathrm{E} \quad 11$ & $2.19 \mathrm{E}-11$ & $6.46 \mathrm{E}-11$ & $5.80 \mathrm{E}-11$ & $1.07 \mathrm{E}-10$ \\
\hline 1.3 & $3.00 \mathrm{E}-10$ & $1.94 \mathrm{~F}-11$ & $6.44 \mathrm{E}-12$ & $1.92 \mathrm{E}-11$ & $6.03 \mathrm{E}-11$ & $1.95 \mathrm{E}-11$ & $5.76 \mathrm{E}-11$ & $5.17 \mathrm{E}-11$ & $9.53 \mathrm{E}-11$ \\
\hline 1.4 & $2.67 \mathrm{E}-10$ & $1.73 \mathrm{E}-11$ & $5.74 \mathrm{E}-12$ & $1.71 \mathrm{E}-11$ & $5.38 \mathrm{E}-11$ & $1.74 \mathrm{E}-11$ & $5.13 \mathrm{E}-11$ & $4.61 \mathrm{E}-11$ & $8.50 \mathrm{E}-11$ \\
\hline 1.5 & $2.38 \mathrm{E}-10$ & $1.54 \mathrm{E}-11$ & $5.11 \mathrm{E}-12$ & $1.53 \mathrm{E}-11$ & $4.79 \mathrm{E}-11$ & $1.55 \mathrm{E}-11$ & $4.58 \mathrm{E}-11$ & $4.11 \mathrm{E}-11$ & $7.57 \mathrm{E}-11$ \\
\hline 1.6 & $2.12 \mathrm{E}-10$ & $1.37 \mathrm{E}-11$ & $4.56 \mathrm{E}-12$ & $1.36 \mathrm{E}-11$ & $4.27 \mathrm{E}-11$ & $1.38 \mathrm{E}-11$ & $4.08 \mathrm{E}-11$ & $3.66 \mathrm{E}-11$ & $6.75 \mathrm{E}-11$ \\
\hline 1.7 & $1.89 \mathrm{E}-10$ & $1.22 \mathrm{E}-11$ & $4.06 \mathrm{E}-12$ & $1.21 \mathrm{E}-11$ & $3.81 \mathrm{E}-11$ & $1.23 \mathrm{E}-11$ & $3.63 \mathrm{E}-11$ & $3.26 \mathrm{E}-11$ & $6.02 \mathrm{E}-11$ \\
\hline 1.8 & $1.68 \mathrm{E}-10$ & $1.09 \mathrm{E}-11$ & $3.62 \mathrm{E}-12$ & $1.08 \mathrm{E}-11$ & $3.39 \mathrm{E}-11$ & $1.10 \mathrm{E}-11$ & $3.24 \mathrm{E}-11$ & $2.91 \mathrm{E}-11$ & $5.36 \mathrm{E}-11$ \\
\hline 1.9 & $1.50 \mathrm{E}-10$ & $9.73 \mathrm{E}-12$ & $3.23 \mathrm{E}-12$ & $9.62 \mathrm{E}-12$ & $3.02 \mathrm{E}-11$ & $9.79 \mathrm{E}-12$ & $2.89 \mathrm{E}-11$ & $2.59 \mathrm{E}-11$ & $4.78 \mathrm{E}-11$ \\
\hline 2.0 & $1.34 \mathrm{E}-10$ & $8.67 \mathrm{E}-12$ & $2.88 \mathrm{E}-12$ & $8.58 \mathrm{E}-12$ & $2.69 \mathrm{E}-11$ & $8.73 \mathrm{E}-12$ & $2.57 \mathrm{E}-11$ & $2.31 \mathrm{E}-11$ & $4.26 \mathrm{E}-11$ \\
\hline 2.1 & $1.19 \mathrm{E}-10$ & $7.73 \mathrm{E}-12$ & $2.56 \mathrm{E}-12$ & $7.65 \mathrm{E}-12$ & $2.40 \mathrm{E}-11$ & $7.78 \mathrm{E}-12$ & $2.29 \mathrm{E}-11$ & $2.06 \mathrm{E}-11$ & $3.80 \mathrm{E}-11$ \\
\hline 2.2 & $1.06 \mathrm{E}-10$ & 6.89E -12 & $2.28 \mathrm{E}-12$ & $6.81 \mathrm{E}-12$ & $2.14 \mathrm{E}-11$ & $6.93 \mathrm{E}-12$ & $2.04 \mathrm{E}-11$ & $1.84 \mathrm{E}-11$ & $3.38 \mathrm{E}-11$ \\
\hline 2.3 & $9.47 \mathrm{E}-11$ & $6.14 \mathrm{E}-12$ & $2.04 \mathrm{E}-12$ & $6.07 \mathrm{E}-12$ & $1.91 \mathrm{E}-11$ & $6.18 \mathrm{E}-12$ & $1.82 \mathrm{E}-11$ & $1.64 \mathrm{E}-11$ & $3.01 \mathrm{E}-11$ \\
\hline 2.4 & $8.44 \mathrm{E}-11$ & $5.47 \mathrm{E}-12$ & $1.81 \mathrm{E}-12$ & $5.41 \mathrm{E}-12$ & $1.70 \mathrm{E}-11$ & $5.51 \mathrm{E}-12$ & $1.62 \mathrm{E}-11$ & $1.46 \mathrm{E}-11$ & $2.69 \mathrm{E}-11$ \\
\hline 2.5 & $7.52 \mathrm{E}-11$ & 4.87E -12 & $1.62 \mathrm{E}-12$ & $4.82 \mathrm{E}-12$ & $1.52 \mathrm{E}-11$ & $4.91 \mathrm{E}-12$ & $1.45 \mathrm{E}-11$ & $1.30 \mathrm{E}-11$ & $2.39 \mathrm{E}-11$ \\
\hline 2.6 & $6.70 \mathrm{E}-11$ & $4.34 \mathrm{E}-12$ & $1.44 \mathrm{E}-12$ & $4.30 \mathrm{E}-12$ & $1.35 \mathrm{E}-11$ & $4.37 \mathrm{E}-12$ & $1.29 \mathrm{E}-11$ & $1.16 \mathrm{E}-11$ & $2.13 \mathrm{E}-11$ \\
\hline 2.7 & $5.98 \mathrm{E}-11$ & $3.87 \mathrm{E}-12$ & $1.28 \mathrm{E}-12$ & $3.83 \mathrm{E}-12$ & $1.20 \mathrm{E}-11$ & $3.90 \mathrm{E}-12$ & $1.15 \mathrm{E}-11$ & $1.03 \mathrm{E}-11$ & $1.90 \mathrm{E} \quad 11$ \\
\hline 2.8 & $5.33 \mathrm{E}-11$ & $3.45 \mathrm{E}-12$ & $1.14 \mathrm{E}-12$ & $3.41 \mathrm{E}-12$ & $1.07 \mathrm{E}-11$ & $3.47 \mathrm{E}-12$ & $1.02 \mathrm{E}-11$ & $9.20 \mathrm{E}-12$ & $1.70 \mathrm{E}-11$ \\
\hline 2.9 & $4.75 \mathrm{E}-11$ & $3.08 \mathrm{E}-12$ & $1.02 \mathrm{E}-12$ & $3.04 \mathrm{E}-12$ & $9.56 \mathrm{E}-12$ & $3.10 \mathrm{E}-12$ & $9.13 \mathrm{E}-12$ & $8.20 \mathrm{E}-12$ & $1.51 \mathrm{E}-11$ \\
\hline 3.0 & $4.23 \mathrm{E}-11$ & $2.74 \mathrm{E}-12$ & $9.09 \mathrm{E}-13$ & $2.71 \mathrm{E}-12$ & $8.52 \mathrm{E}-12$ & $2.76 \mathrm{E}-12$ & $8.13 \mathrm{E}-12$ & $7.31 \mathrm{E}-12$ & $1.35 \mathrm{E}-11$ \\
\hline 3.1 & $3.77 \mathrm{E}-11$ & $2.44 \mathrm{E}-12$ & $8.10 \mathrm{E}-13$ & $2.42 \mathrm{E}-12$ & $7.59 \mathrm{E}-12$ & $2.46 \mathrm{E}-12$ & $7.25 \mathrm{E}-12$ & $6.51 \mathrm{E}-12$ & $1.20 \mathrm{E}-11$ \\
\hline 3.2 & $3.36 \mathrm{E}-11$ & $2.18 \mathrm{E}-12$ & $7.22 \mathrm{E}-13$ & $2.15 \mathrm{E}-12$ & $6.77 \mathrm{E}-12$ & $2.19 \mathrm{E}-12$ & $6.46 \mathrm{E}-12$ & $5.80 \mathrm{E}-12$ & $1.07 \mathrm{E}-11$ \\
\hline 3.3 & $2.99 \mathrm{E}-11$ & $1.94 \mathrm{E}-12$ & $6.44 \mathrm{E}-13$ & $1.92 \mathrm{E}-12$ & $6.03 \mathrm{E}-12$ & $1.95 \mathrm{E}-12$ & $5.76 \mathrm{E}-12$ & $5.17 \mathrm{E}-12$ & $9.53 \mathrm{E}-12$ \\
\hline 3.4 & $2.67 \mathrm{E}-11$ & $1.73 \mathrm{E}-12$ & $5.74 \mathrm{E}-13$ & $1.71 \mathrm{E}-12$ & $5.38 \mathrm{E}-12$ & $1.74 \mathrm{E}-12$ & $5.13 \mathrm{E}-12$ & $4.61 \mathrm{E}-12$ & $8.49 \mathrm{E}-12$ \\
\hline 3.5 & $2.38 \mathrm{E}-11$ & $1.54 \mathrm{E}-12$ & $5.11 \mathrm{E}-13$ & $1.52 \mathrm{E}-12$ & $4.79 \mathrm{E}-12$ & $1.55 \mathrm{E}-12$ & 4.57E -12 & $4.11 \mathrm{E}-12$ & 7.57E $\quad 12$ \\
\hline 3.6 & $2.12 \mathrm{E}-11$ & $1.37 \mathrm{E}-12$ & $4.56 \mathrm{E}-13$ & $1.36 \mathrm{E}-12$ & $4.27 \mathrm{E}-12$ & $1.38 \mathrm{E}-12$ & $4.07 \mathrm{E}-12$ & $3.66 \mathrm{E}-12$ & $6.74 \mathrm{E}-12$ \\
\hline 3.7 & $1.89 \mathrm{E}-11$ & $1.22 \mathrm{E}-12$ & $4.06 \mathrm{E}-13$ & $1.21 \mathrm{E}-12$ & $3.81 \mathrm{E}-12$ & $1.23 \mathrm{E}-12$ & $3.63 \mathrm{E}-12$ & $3.26 \mathrm{E}-12$ & $6.01 \mathrm{E}-12$ \\
\hline 3.8 & $1.68 \mathrm{E}-11$ & $1.09 \mathrm{E}-12$ & $3.62 \mathrm{E}-13$ & $1.08 \mathrm{E}-12$ & $3.39 \mathrm{E}-12$ & $1.10 \mathrm{E}-12$ & $3.24 \mathrm{~F}-12$ & $2.91 \mathrm{E}-12$ & $5.36 \mathrm{E}-12$ \\
\hline 3.9 & $1.50 \mathrm{E}-11$ & $9.73 \mathrm{E}-13$ & $3.23 \mathrm{E}-13$ & $9.61 \mathrm{E} \quad 13$ & $3.02 \mathrm{E} \quad 12$ & $9.79 \mathrm{E}-13$ & $2.88 \mathrm{E}-12$ & $2.59 \mathrm{E}-12$ & $4.77 \mathrm{E}-12$ \\
\hline 4.0 & $1.34 \mathrm{E}-11$ & $8.67 \mathrm{E}-13$ & $2.88 \mathrm{E}-13$ & $8.56 \mathrm{E}-13$ & $2.69 \mathrm{E}-12$ & $8.73 \mathrm{E}-13$ & $2.57 \mathrm{E}-12$ & $2.31 \mathrm{E}-12$ & $4.25 \mathrm{E}-12$ \\
\hline 4.1 & $1.19 \mathrm{E}-11$ & $7.72 \mathrm{E}-13$ & $2.56 \mathrm{E}-13$ & $7.63 \mathrm{E}-13$ & $2.40 \mathrm{E}-12$ & $7.78 \mathrm{E}-13$ & $2.29 \mathrm{E}-12$ & $2.05 \mathrm{E}-12$ & $3.79 \mathrm{E}-12$ \\
\hline 4.2 & $1.06 \mathrm{E}-11$ & $6.88 \mathrm{E}-13$ & $2.28 \mathrm{E}-13$ & $6.80 \mathrm{E}-13$ & $2.14 \mathrm{E}-12$ & $6.93 \mathrm{E}-13$ & $2.04 \mathrm{E}-12$ & $1.83 \mathrm{E}-12$ & $3.37 \mathrm{E}-12$ \\
\hline 4.3 & $9.47 \mathrm{E}-12$ & $6.14 \mathrm{E}-13$ & $2.04 \mathrm{E}-13$ & $6.05 \mathrm{E}-13$ & $1.91 \mathrm{E}-12$ & $6.18 \mathrm{E}-13$ & $1.82 \mathrm{E}-12$ & $1.63 \mathrm{E}-12$ & $3.00 \mathrm{E}-12$ \\
\hline 4.4 & $8.44 \mathrm{E}-12$ & $5.47 \mathrm{E}-13$ & $1.81 \mathrm{E}-13$ & $5.39 \mathrm{E}-13$ & $1.70 \mathrm{~F}-12$ & $5.51 \mathrm{E}-13$ & $1.62 \mathrm{E}-12$ & $1.45 \mathrm{E}-12$ & $2.68 \mathrm{E}-12$ \\
\hline 4.5 & $7.52 \mathrm{E}-12$ & $4.87 \mathrm{E}-13$ & $1.62 \mathrm{E}-13$ & $4.80 \mathrm{E}-13$ & $1.52 \mathrm{E}-12$ & $4.91 \mathrm{E}-13$ & $1.44 \mathrm{E}-12$ & $1.29 \mathrm{E}-12$ & $2.38 \mathrm{E}-12$ \\
\hline 4.6 & $6.70 \mathrm{E}-12$ & $4.34 \mathrm{E}-13$ & $1.44 \mathrm{E}-13$ & $4.27 \mathrm{E}-13$ & $1.35 \mathrm{E}-12$ & $4.37 \mathrm{E}-13$ & $1.28 \mathrm{E}-12$ & $1.15 \mathrm{E}-12$ & $2.12 \mathrm{E}-12$ \\
\hline 4.7 & $5.97 \mathrm{E}-12$ & $3.87 \mathrm{E}-13$ & $1.28 \mathrm{E}-13$ & $3.80 \mathrm{E}-13$ & $1.20 \mathrm{E}-12$ & $3.90 \mathrm{E}-13$ & $1.14 \mathrm{E}-12$ & $1.02 \mathrm{E}-12$ & $1.89 \mathrm{E}-12$ \\
\hline 4.8 & $5.32 \mathrm{E}-12$ & $3.45 \mathrm{E}-13$ & $1.14 \mathrm{E}-13$ & $3.38 \mathrm{E}-13$ & $1.07 \mathrm{E}-12$ & $3.48 \mathrm{E}-13$ & $1.01 \mathrm{E}-12$ & $9.09 \mathrm{E}-13$ & $1.68 \mathrm{E}-12$ \\
\hline 4.9 & $4.74 \mathrm{E}-12$ & $3.07 \mathrm{E}-13$ & $1.02 \mathrm{E}-13$ & $3.00 \mathrm{E}-13$ & $9.56 \mathrm{E}-13$ & $3.10 \mathrm{E}-13$ & $9.00 \mathrm{E}-13$ & $8.08 \mathrm{E}-13$ & $1.49 \mathrm{E}-12$ \\
\hline 5.0 & $4.22 \mathrm{E} \quad 12$ & $2.74 \mathrm{E}-13$ & $9.10 \mathrm{E}-14$ & $2.67 \mathrm{E}-13$ & $8.52 \mathrm{E}-13$ & $2.76 \mathrm{E}-13$ & $8.00 \mathrm{E}-13$ & $7.17 \mathrm{E}-13$ & $1.32 \mathrm{E}-12$ \\
\hline 5.1 & $3.76 \mathrm{E}-12$ & $2.44 \mathrm{E}-13$ & $8.11 \mathrm{E}-14$ & $2.37 \mathrm{E}-13$ & $7.59 \mathrm{E}-13$ & $2.46 \mathrm{E}-13$ & $7.10 \mathrm{E} \quad 13$ & $6.36 \mathrm{E}-13$ & $1.17 \mathrm{E}-12$ \\
\hline 5.2 & $3.35 \mathrm{E}-12$ & $2.18 \mathrm{E}-13$ & $7.23 \mathrm{E}-14$ & $2.10 \mathrm{E}-13$ & $6.77 \mathrm{E}-13$ & $2.19 \mathrm{E}-13$ & $6.29 \mathrm{E}-13$ & $5.64 \mathrm{~L}-13$ & $1.04 \mathrm{E}-12$ \\
\hline 5.3 & $2.98 \mathrm{E}-12$ & $1.94 \mathrm{E}-13$ & $6.44 \mathrm{E}-14$ & $1.86 \mathrm{E}-13$ & $6.03 \mathrm{E}-13$ & $1.95 \mathrm{E}-13$ & $5.57 \mathrm{E}-13$ & $4.99 \mathrm{E}-13$ & $9.22 \mathrm{E}-13$ \\
\hline 5.4 & $2.66 \mathrm{E}-12$ & $1.73 \mathrm{E}-13$ & $5.74 \mathrm{E}-14$ & $1.64 \mathrm{E}-13$ & 5.37E 13 & $1.74 \mathrm{E} \quad 13$ & $4.92 \mathrm{E}-13$ & $4.41 \mathrm{E}-13$ & $8.15 \mathrm{E}-13$ \\
\hline 5.5 & $2.36 \mathrm{E}-12$ & $1.54 \mathrm{E}-13$ & $5.11 \mathrm{E}-14$ & $1.45 \mathrm{E}-13$ & $4.79 \mathrm{E}-13$ & $1.55 \mathrm{E}-13$ & $4.34 \mathrm{E}-13$ & $3.88 \mathrm{E}-13$ & $7.19 \mathrm{E}-13$ \\
\hline 5.6 & $2.10 \mathrm{E}-12$ & $1.37 \mathrm{E}-13$ & $4.55 \mathrm{E}-14$ & $1.27 \mathrm{E}-13$ & $4.26 \mathrm{E}-13$ & $1.38 \mathrm{E}-13$ & $3.82 \mathrm{E}-13$ & $3.41 \mathrm{E}-13$ & $6.33 \mathrm{E}-13$ \\
\hline 5.7 & $1.87 \mathrm{E}-12$ & $1.22 \mathrm{E}-13$ & $4.05 \mathrm{E}-14$ & $1.12 \mathrm{E}-13$ & $3.80 \mathrm{E}-13$ & $1.23 \mathrm{E}-13$ & $3.35 \mathrm{E}-13$ & $2.99 \mathrm{E}-13$ & $5.55 \mathrm{E}-13$ \\
\hline 5.8 & $1.66 \mathrm{E}-12$ & $1.08 \mathrm{E}-13$ & $3.60 \mathrm{E}-14$ & $9.79 \mathrm{E}-14$ & $3.38 \mathrm{E}-13$ & $1.10 \mathrm{E}-13$ & $2.93 \mathrm{E}-13$ & $2.61 E-13$ & $4.86 \mathrm{E}-13$ \\
\hline 5.9 & $1.48 \mathrm{E}-12$ & $9.64 \mathrm{E}-14$ & $3.20 \mathrm{E}-14$ & $8.53 \mathrm{E}-14$ & $3.01 \mathrm{E}-13$ & $9.74 \mathrm{E}-14$ & $2.56 \mathrm{E}-13$ & $2.27 \mathrm{E}-13$ & $4.23 \mathrm{E}-13$ \\
\hline 6.0 & $1.31 \mathrm{E}-12$ & $8.57 \mathrm{E}-14$ & $2.84 \mathrm{~F}-14$ & $7.40 \mathrm{E}-14$ & $2.67 \mathrm{E}-13$ & $8.66 \mathrm{E}-14$ & $2.22 \mathrm{E}-13$ & $1.96 \mathrm{E}-13$ & $3.67 \mathrm{E}-13$ \\
\hline 6.1 & 1.16E -12 & $7.60 \mathrm{E}-14$ & $2.52 \mathrm{E}-14$ & $6.39 \mathrm{E}-14$ & $2.38 \mathrm{E}-13$ & $7.68 \mathrm{E}-14$ & $1.91 \mathrm{E}-13$ & $1.69 \mathrm{E}-13$ & $3.17 \mathrm{E}-13$ \\
\hline 6.2 & $1.03 \mathrm{E}-12$ & $6.76 \mathrm{E}-14$ & $2.23 \mathrm{E}-14$ & $5.48 \mathrm{E}-14$ & $2.11 \mathrm{E}-13$ & $6.80 \mathrm{E}-14$ & $1.64 \mathrm{E}-13$ & $1.45 \mathrm{E}-13$ & $2.72 \mathrm{E}-13$ \\
\hline 6.3 & $9.09 \mathrm{E}-13$ & $6.10 \mathrm{E}-14$ & $1.98 \mathrm{E}-14$ & $4.67 \mathrm{E}-14$ & $1.89 \mathrm{E}-13$ & $6.01 \mathrm{E}-14$ & $1.40 \mathrm{E}-13$ & $1.28 \mathrm{E}-13$ & $2.32 \mathrm{E}-13$ \\
\hline 6.4 & $8.01 \mathrm{E}-13$ & $5.72 \mathrm{E}-14$ & $1.79 \mathrm{E}-14$ & $3.96 \mathrm{E}-14$ & $1.73 \mathrm{E}-13$ & $5.31 \mathrm{E}-14$ & $1.19 \mathrm{E}-13$ & $1.19 \mathrm{E}-13$ & $1.98 \mathrm{E}-13$ \\
\hline 6.5 & $7.05 \mathrm{E}-13$ & $5.72 \mathrm{E}-14$ & $1.71 \mathrm{E}-14$ & $3.33 \mathrm{E}-14$ & $1.64 \mathrm{E}-13$ & $4.69 \mathrm{E}-14$ & $1.01 \mathrm{E}-13$ & $1.23 \mathrm{E}-13$ & $1.69 \mathrm{E}-13$ \\
\hline 6.6 & $6.19 \mathrm{E}-13$ & $6.07 \mathrm{E}-14$ & $1.76 \mathrm{E}-14$ & $2.78 \mathrm{E}-14$ & $1.61 \mathrm{E}-13$ & $4.15 \mathrm{E}-14$ & $8.63 \mathrm{E}-14$ & $1.38 \mathrm{E}-13$ & $1.45 \mathrm{E}-13$ \\
\hline 6.7 & $5.41 \mathrm{E}-13$ & $6.60 \mathrm{E}-14$ & $1.92 \mathrm{E}-14$ & $2.31 \mathrm{E}-14$ & $1.61 \mathrm{E}-13$ & $3.69 \mathrm{E}-14$ & $7.40 \mathrm{E}-14$ & $1.58 \mathrm{E}-13$ & $1.25 \mathrm{E}-13$ \\
\hline 6.8 & $4.71 \mathrm{E}-13$ & $7.04 \mathrm{E}-14$ & $2.12 \mathrm{E}-14$ & $1.91 \mathrm{E}-14$ & $1.60 \mathrm{E}-13$ & $3.28 \mathrm{E}-14$ & $6.38 \mathrm{E}-14$ & $1.75 \mathrm{E}-13$ & $1.09 \mathrm{E}-13$ \\
\hline 6.9 & $4.08 \mathrm{E}-13$ & $7.22 \mathrm{E}-14$ & $2.27 \mathrm{E}-14$ & $1.56 \mathrm{E}-14$ & $1.55 \mathrm{E}-13$ & $2.91 \mathrm{E} \quad 14$ & $5.49 \mathrm{E}-14$ & $1.84 \mathrm{E}-13$ & $9.41 \mathrm{E}-14$ \\
\hline 7.0 & $3.52 \mathrm{E}-13$ & $7.04 \mathrm{E}-14$ & $2.31 \mathrm{E}-14$ & $1.27 \mathrm{E}-14$ & $1.45 \mathrm{E}-13$ & $2.56 \mathrm{E}-14$ & $4.68 \mathrm{E}-14$ & $1.80 \mathrm{E}-13$ & $8.05 \mathrm{E}-14$ \\
\hline 7.1 & $3.01 \mathrm{E} \quad 13$ & $6.53 \mathrm{E}-14$ & $2.22 \mathrm{E}-14$ & $1.03 \mathrm{E}-14$ & $1.31 \mathrm{E}-13$ & $2.22 \mathrm{E}-14$ & $3.94 \mathrm{E}-14$ & $1.67 \mathrm{E}-13$ & $6.79 \mathrm{E}-14$ \\
\hline 7.2 & $2.56 \mathrm{E}-13$ & $5.79 \mathrm{E}-14$ & $2.03 \mathrm{E}-14$ & $8.20 \mathrm{E}-15$ & $1.15 \mathrm{E}-13$ & $1.89 \mathrm{E}-14$ & $3.26 \mathrm{E}-14$ & $1.48 \mathrm{E}-13$ & $5.62 \mathrm{E}-14$ \\
\hline 7.3 & $2.16 \mathrm{E}-13$ & $4.95 \mathrm{E}-14$ & $1.78 \mathrm{E}-14$ & $6.50 \mathrm{E}-15$ & $9.71 \mathrm{E}-14$ & $1.59 \mathrm{E}-14$ & $2.66 \mathrm{E}-14$ & $1.25 \mathrm{E}-13$ & $4.57 \mathrm{E}-14$ \\
\hline 7.4 & $1.81 \mathrm{E}-13$ & $4.10 \mathrm{E} \quad 14$ & $1.50 \mathrm{E}-14$ & $5.10 \mathrm{E}-15$ & $8.01 \mathrm{E} \quad 14$ & $1.32 \mathrm{E}-14$ & $2.13 \mathrm{E}-14$ & $1.02 \mathrm{E}-13$ & $3.65 \mathrm{E}-14$ \\
\hline 7.5 & $1.51 \mathrm{E}-13$ & $3.31 \mathrm{E}-14$ & $1.22 \mathrm{E}-14$ & $3.98 \mathrm{E}-15$ & $6.48 \mathrm{E}-14$ & $1.08 \mathrm{E}-14$ & $1.68 \mathrm{E}-14$ & $8.14 \mathrm{E}-14$ & $2.87 \mathrm{E}-14$ \\
\hline 7.6 & $1.24 \mathrm{E}-13$ & $2.62 \mathrm{E}-14$ & $9.76 \mathrm{E}-15$ & $3.08 \mathrm{E}-15$ & $5.14 \mathrm{E}-14$ & $8.66 \mathrm{E}-15$ & $1.30 \mathrm{E}-14$ & $6.33 \mathrm{E}-14$ & $2.23 \mathrm{E}-14$ \\
\hline 7.7 & $1.01 \mathrm{E}-13$ & $2.04 \mathrm{E}-14$ & $7.62 \mathrm{E}-15$ & $2.37 \mathrm{E}-15$ & $4.03 \mathrm{E}-14$ & $6.90 \mathrm{E}-15$ & $1.00 \mathrm{E}-14$ & $4.84 \mathrm{E}-14$ & $1.72 \mathrm{E}-14$ \\
\hline 7.8 & $8.23 \mathrm{E}-14$ & $1.56 \mathrm{E}-14$ & $5.86 \mathrm{E}-15$ & $1.81 \mathrm{E}-15$ & $3.12 \mathrm{E}-14$ & $5.44 \mathrm{E}-15$ & $7.66 \mathrm{E}-15$ & $3.65 \mathrm{E}-14$ & $1.31 \mathrm{E}-14$ \\
\hline 7.9 & $6.62 \mathrm{E}-14$ & $1.19 \mathrm{E}-14$ & $4.45 \mathrm{E}-15$ & $1.38 \mathrm{E}-15$ & $2.39 \mathrm{E}-14$ & $4.26 \mathrm{E}-15$ & $5.80 \mathrm{E}-15$ & $2.72 \mathrm{E}-14$ & $9.88 \mathrm{E}-15$ \\
\hline 8.0 & $5.29 \mathrm{E}-14$ & $8.95 \mathrm{E}-15$ & $3.35 \mathrm{E}-15$ & $1.04 \mathrm{E}-15$ & $1.82 \mathrm{E}-14$ & $3.30 \mathrm{E}-15$ & $4.36 \mathrm{E}-15$ & $2.01 \mathrm{E}-14$ & $7.42 \mathrm{E}-15$ \\
\hline 8.1 & $4.20 \mathrm{E}-14$ & $6.70 \mathrm{E}-15$ & $2.50 \mathrm{E}-15$ & $7.87 \mathrm{E}-16$ & $1.38 \mathrm{E}-14$ & $2.55 \mathrm{E}-15$ & $3.26 \mathrm{E}-15$ & $1.47 \mathrm{E}-14$ & $5.54 \mathrm{E}-15$ \\
\hline 8.2 & $3.31 \mathrm{E}-14$ & $4.98 \mathrm{E}-15$ & $1.85 \mathrm{E}-15$ & $5.92 \mathrm{E}-16$ & $1.04 \mathrm{E}-14$ & $1.95 \mathrm{E}-15$ & $2.43 \mathrm{E}-15$ & $1.07 \mathrm{E}-14$ & $4.13 \mathrm{E}-15$ \\
\hline
\end{tabular}


Table 4 (continued)

\begin{tabular}{|c|c|c|c|c|c|c|c|c|c|}
\hline \multirow[t]{2}{*}{$\log _{10} T(\mathrm{~K})$} & \multicolumn{9}{|c|}{$x_{R}\left(\mathrm{~cm}^{3} \mathrm{~s}^{-1}\right)$} \\
\hline & $1 \mathrm{~s}^{21} \mathrm{~S}$ & $1 \mathrm{~s} 2 \mathrm{~s}^{1} \mathrm{~S}_{0}$ & $1 \mathrm{~s}^{2} \mathrm{~s}^{1} \mathrm{~S}_{0}$ & $1 \mathrm{~s} 2 \mathrm{p}^{3} \mathrm{P}_{0}^{0}$ & $1 s 2 s^{3} S_{1}$ & $1 \mathrm{~s} 3 \mathrm{~s}^{3} \mathrm{~S}_{1}$ & $1 \mathrm{~s} 2 \mathrm{p}^{3} \mathrm{P}_{1}^{\mathrm{o}}$ & $1 \mathrm{~s} 2 \mathrm{p}^{1} \mathrm{P}_{1}^{\mathrm{o}}$ & $1 \mathrm{~s} 2 \mathrm{p}^{3} \mathrm{P}_{2}^{\mathrm{o}}$ \\
\hline & \multicolumn{9}{|l|}{ Al XII } \\
\hline BE: & -153.0 & 36.5 & -16.2 & 36.6 & 37.6 & -16.4 & 36.6 & -35.8 & 36.5 \\
\hline 8.3 & $2.60 \mathrm{E} \quad 14$ & $3.69 \mathrm{E}-15$ & $1.37 \mathrm{E}-15$ & 4.44E $\quad 16$ & $7.76 \mathrm{E}-15$ & $1.49 \mathrm{E}-15$ & $1.81 \mathrm{E} \quad 15$ & $7.79 \mathrm{E}-15$ & $3.06 \mathrm{E}-15$ \\
\hline 8.4 & $2.02 \mathrm{E}-14$ & $2.73 \mathrm{E}-15$ & $1.00 \mathrm{E}-15$ & $3.32 \mathrm{E}-16$ & $5.79 \mathrm{E}-15$ & $1.13 \mathrm{E}-15$ & $1.34 \mathrm{E}-15$ & $5.63 \mathrm{E}-15$ & $2.26 \mathrm{E}-15$ \\
\hline 8.5 & $1.57 \mathrm{E}-14$ & $2.01 \mathrm{E}-15$ & $7.35 \mathrm{E}-16$ & $2.47 \mathrm{E}-16$ & $4.31 \mathrm{E}-15$ & $8.53 \mathrm{E}-16$ & $9.88 \mathrm{E}-16$ & $4.06 \mathrm{E}-15$ & $1.67 \mathrm{E}-15$ \\
\hline 8.6 & $1.21 \mathrm{E}-14$ & $1.47 \mathrm{E}-15$ & $5.36 \mathrm{E}-16$ & $1.84 \mathrm{E}-16$ & $3.20 \mathrm{E}-15$ & $6.43 \mathrm{E}-16$ & $7.28 \mathrm{E}-16$ & $2.92 \mathrm{E}-15$ & $1.23 \mathrm{E}-15$ \\
\hline 8.7 & $9.28 \mathrm{E}-15$ & $1.08 \mathrm{E}-15$ & $3.91 \mathrm{E}-16$ & $1.37 \mathrm{E}-16$ & $2.37 \mathrm{E}-15$ & $4.82 \mathrm{E}-16$ & $5.35 \mathrm{E}-16$ & $2.10 \mathrm{E}-15$ & $9.03 \mathrm{E}-16$ \\
\hline 8.8 & $7.09 \mathrm{E}-15$ & $7.91 \mathrm{E}-16$ & $2.84 \mathrm{E}-16$ & $1.01 \mathrm{E}-16$ & $1.75 \mathrm{E}-1.5$ & $3.61 \mathrm{E}-16$ & $3.93 \mathrm{E}-16$ & $1.51 \mathrm{E}-15$ & $6.62 \mathrm{E}-16$ \\
\hline 8.9 & $5.39 \mathrm{E} \quad 15$ & $5.78 \mathrm{E}-16$ & $2.06 \mathrm{E}-16$ & $7.49 \mathrm{E}-17$ & $1.29 \mathrm{E}-15$ & $2.69 \mathrm{E}-16$ & $2.88 \mathrm{E}-16$ & $1.08 \mathrm{E}-15$ & $4.85 \mathrm{E}-16$ \\
\hline \multirow[t]{2}{*}{9.0} & $4.09 \mathrm{E}-15$ & $4.22 \mathrm{E}-16$ & $1.50 \mathrm{E}-16$ & $5.53 \mathrm{E}-17$ & $9.51 \mathrm{E}-16$ & $2.00 \mathrm{E}-16$ & $2.11 \mathrm{E}-16$ & $7.73 \mathrm{E}-16$ & $3.55 \mathrm{E}-16$ \\
\hline & \multicolumn{9}{|l|}{ Si XIII } \\
\hline BE: & -179.0 & 42.9 & -19.0 & 43.0 & 44.0 & -19.3 & 42.9 & 42.1 & 42.8 \\
\hline 1.0 & $4.92 \mathrm{E}-10$ & $3.22 \mathrm{E}-11$ & $1.07 \mathrm{E}-11$ & $3.17 \mathrm{E}-11$ & $9.98 \mathrm{E}-11$ & $3.24 \mathrm{E}-11$ & $9.52 \mathrm{E}-11$ & $8.59 \mathrm{E}-11$ & $1.57 \mathrm{E}-10$ \\
\hline 1.1 & $4.39 \mathrm{E}-10$ & $2.87 \mathrm{E}-11$ & $9.53 \mathrm{E}-12$ & $2.83 \mathrm{E}-11$ & $8.90 \mathrm{E}-11$ & $2.89 \mathrm{E}-11$ & $8.49 \mathrm{E}-11$ & $7.66 \mathrm{E}-11$ & $1.40 \mathrm{E}-10$ \\
\hline 1.2 & $3.91 \mathrm{E}-10$ & $2.56 \mathrm{E}-11$ & $8.49 \mathrm{E}-12$ & $2.52 \mathrm{E}-11$ & $7.93 \mathrm{E}-11$ & $2.57 \mathrm{E}-11$ & $7.56 \mathrm{E}-11$ & $6.82 \mathrm{E}-11$ & $1.25 \mathrm{E}-10$ \\
\hline 1.3 & $3.49 \mathrm{E}-10$ & $2.28 \mathrm{E}-11$ & $7.57 \mathrm{E}-12$ & $2.25 \mathrm{E}-11$ & $7.07 \mathrm{E}-11$ & $2.29 \mathrm{E}-11$ & $6.74 \mathrm{E}-11$ & $6.08 \mathrm{E}-11$ & $1.11 \mathrm{E}-10$ \\
\hline 1.4 & $3.11 \mathrm{E}-10$ & $2.03 \mathrm{E}-11$ & $6.74 \mathrm{E}-12$ & $2.00 \mathrm{E}-11$ & $6.30 \mathrm{E}-11$ & $2.04 \mathrm{E}-11$ & $6.01 \mathrm{E}-11$ & $5.42 \mathrm{E}-11$ & $9.93 \mathrm{E}-11$ \\
\hline 1.5 & $2.77 \mathrm{E}-10$ & $1.81 \mathrm{E}-11$ & $6.01 \mathrm{E}-12$ & $1.78 \mathrm{E}-11$ & $5.61 \mathrm{E}-11$ & $1.82 \mathrm{E}-11$ & $5.35 \mathrm{E}-11$ & $4.83 \mathrm{E}-11$ & $8.85 \mathrm{E}-11$ \\
\hline 1.6 & 2.47E 10 & $1.61 \mathrm{E}-11$ & $5.36 \mathrm{E}-12$ & $1.59 \mathrm{E}-11$ & $5.00 \mathrm{E}-11$ & $1.62 \mathrm{E}-11$ & 4.77E 11 & $4.31 \mathrm{E}-11$ & 7.89E 11 \\
\hline 1.7 & $2.20 \mathrm{E} \quad 10$ & $1.44 \mathrm{E}-11$ & 4.77t -12 & $1.42 \mathrm{E}-11$ & $4.46 \mathrm{E}-11$ & $1.45 \mathrm{E}-11$ & $4.25 \mathrm{E}-11$ & $3.84 \mathrm{E}-11$ & $7.03 \mathrm{E}-11$ \\
\hline 1.8 & $1.96 \mathrm{E}-10$ & $1.28 \mathrm{E}-11$ & $4.26 \mathrm{E}-12$ & $1.26 \mathrm{E}-11$ & $3.97 \mathrm{E}-11$ & $1.29 \mathrm{E}-11$ & $3.79 \mathrm{E}-11$ & $3.42 \mathrm{E}-11$ & $6.26 \mathrm{E}-11$ \\
\hline 1.9 & $1.75 \mathrm{E} \quad 10$ & $1.14 \mathrm{E}-11$ & $3.79 \mathrm{E}-12$ & $1.13 \mathrm{E}-11$ & $3.54 \mathrm{E}-11$ & $1.15 \mathrm{E}-11$ & $3.38 \mathrm{E}-11$ & $3.05 \mathrm{E}-11$ & $5.58 \mathrm{E}-11$ \\
\hline 2.0 & $1.56 \mathrm{E}-10$ & $1.02 \mathrm{E}-11$ & $3.38 \mathrm{E}-12$ & $1.00 \mathrm{E}-11$ & $3.16 \mathrm{E}-11$ & $1.02 \mathrm{E}-11$ & $3.01 \mathrm{E}-11$ & $2.72 \mathrm{E}-11$ & $4.98 \mathrm{E}-11$ \\
\hline 2.1 & $1.39 \mathrm{E} \quad 10$ & $9.08 \mathrm{E}-12$ & $3.01 \mathrm{E}-12$ & $8.94 \mathrm{E}-12$ & $2.81 \mathrm{E}-11$ & $9.13 \mathrm{E}-12$ & $2.68 \mathrm{E}-11$ & $2.42 \mathrm{E}-11$ & $4.43 \mathrm{E}-11$ \\
\hline 2.2 & $1.24 \mathrm{E}-10$ & $8.09 \mathrm{E}-12$ & $2.68 \mathrm{E}-12$ & $7.97 \mathrm{E}-12$ & $2.51 \mathrm{E}-11$ & $8.14 \mathrm{~F}-12$ & $2.39 \mathrm{E}-11$ & $2.16 \mathrm{E}-11$ & $3.95 \mathrm{E}-11$ \\
\hline 2.3 & $1.10 \mathrm{E}-10$ & $7.21 \mathrm{E}-12$ & $2.39 \mathrm{E}-12$ & $7.10 \mathrm{E}-12$ & $2.23 \mathrm{E}-11$ & $7.26 \mathrm{E}-12$ & $2.13 \mathrm{E}-11$ & $1.92 \mathrm{E}-11$ & $3.52 \mathrm{E}-11$ \\
\hline 2.4 & $9.83 \mathrm{E}-11$ & $6.42 \mathrm{E}-12$ & $2.13 \mathrm{E}-12$ & $6.33 \mathrm{E}-12$ & $1.99 \mathrm{E}-11$ & $6.47 \mathrm{E}-12$ & $1.90 \mathrm{E}-11$ & $1.71 \mathrm{E}-11$ & $3.14 \mathrm{E}-11$ \\
\hline 2.5 & $8.76 \mathrm{E}-11$ & $5.73 \mathrm{E}-12$ & $1.90 \mathrm{E}-12$ & $5.64 \mathrm{E}-12$ & $1.78 \mathrm{E}-11$ & $5.76 \mathrm{E}-12$ & $1.69 \mathrm{E}-11$ & $1.53 \mathrm{E}-11$ & $2.80 \mathrm{E}-11$ \\
\hline 2.6 & $7.80 \mathrm{E}-11$ & $5.10 \mathrm{E} \quad 12$ & $1.69 \mathrm{E}-12$ & $5.03 \mathrm{E}-12$ & $1.58 \mathrm{E}-11$ & $5.14 \mathrm{E} \quad 12$ & $1.51 \mathrm{E}-11$ & $1.36 \mathrm{E}-11$ & $2.49 \mathrm{E}-11$ \\
\hline 2.7 & $6.96 \mathrm{E}-11$ & $4.55 \mathrm{E}-12$ & $1.51 \mathrm{E}-12$ & $4.48 \mathrm{E}-12$ & $1.41 \mathrm{E}-11$ & $4.58 \mathrm{E}-12$ & $1.34 \mathrm{E}-11$ & $1.21 \mathrm{E}-11$ & $2.22 \mathrm{E}-11$ \\
\hline 2.8 & $6.20 \mathrm{E}-11$ & $4.05 \mathrm{E}-12$ & $1.35 \mathrm{E}-12$ & $3.99 \mathrm{E}-12$ & $1.26 \mathrm{E}-11$ & $4.08 \mathrm{E}-12$ & $1.20 \mathrm{E}-11$ & $1.08 \mathrm{E}-11$ & $1.98 \mathrm{E}-11$ \\
\hline 2.9 & $5.53 \mathrm{E}-11$ & $3.61 \mathrm{E}-12$ & $1.20 \mathrm{E}-12$ & $3.56 \mathrm{E}-12$ & $1.12 \mathrm{E}-11$ & $3.64 \mathrm{E}-12$ & $1.07 \mathrm{E}-11$ & $9.64 \mathrm{E}-12$ & $1.77 \mathrm{E}-11$ \\
\hline 3.0 & $4.92 \mathrm{E} \quad 11$ & $3.22 \mathrm{E}-12$ & $1.07 \mathrm{E}-12$ & $3.17 \mathrm{E}-12$ & $9.98 \mathrm{E} \quad 12$ & $3.24 \mathrm{E}-12$ & $9.52 \mathrm{E} \quad 12$ & $8.59 \mathrm{E} \quad 12$ & $1.57 \mathrm{E}-11$ \\
\hline 3.1 & $4.39 \mathrm{E}-11$ & $2.87 \mathrm{~F}-12$ & $9.53 \mathrm{E}-13$ & $2.83 \mathrm{E}-12$ & $8.90 \mathrm{E}-12$ & $2.89 \mathrm{E}-12$ & $8.48 \mathrm{E}-12$ & $7.66 \mathrm{E}-12$ & $1.40 \mathrm{E}-11$ \\
\hline 3.2 & $3.91 \mathrm{E}-11$ & $2.56 \mathrm{E}-12$ & $8.49 \mathrm{E}-13$ & $2.52 \mathrm{E}-12$ & $7.93 \mathrm{E}-12$ & $2.57 \mathrm{E}-12$ & $7.56 \mathrm{E}-12$ & $6.82 \mathrm{E}-12$ & $1.25 \mathrm{E}-11$ \\
\hline 3.3 & $3.49 \mathrm{E}-11$ & $2.28 \mathrm{E}-12$ & 7..57E 13 & $2.25 \mathrm{E}-12$ & 7.07E 12 & $2.29 \mathrm{E}-12$ & $6.74 \mathrm{E}-12$ & $6.08 \mathrm{E} \quad 12$ & $1.11 \mathrm{E}-11$ \\
\hline 3.4 & $3.11 \mathrm{E}-11$ & $2.03 \mathrm{E} \quad 12$ & $6.75 \mathrm{E}-13$ & $2.00 \mathrm{E}-12$ & $6.30 \mathrm{E}-12$ & $2.05 \mathrm{E}-12$ & $6.01 \mathrm{E} \quad 12$ & $5.42 \mathrm{E}-12$ & $9.92 \mathrm{E}-12$ \\
\hline 3.5 & $2.77 \mathrm{E}-11$ & $1.81 \mathrm{E}-12$ & $6.01 \mathrm{E}-13$ & $1.78 \mathrm{E}-12$ & $5.61 \mathrm{E}-12$ & $1.82 \mathrm{E}-12$ & $5.35 \mathrm{E}-12$ & $4.83 \mathrm{E}-12$ & $8.84 \mathrm{E}-12$ \\
\hline 3.6 & 2.47E $\quad 11$ & $1.61 \mathrm{E}-12$ & $5.36 \mathrm{E}-13$ & $1.59 \mathrm{E}-12$ & $5.00 \mathrm{E} \quad 12$ & $1.62 \mathrm{E}-12$ & $4.77 \mathrm{E}-12$ & $4.30 \mathrm{E}-12$ & $7.88 \mathrm{E}-12$ \\
\hline 3.7 & $2.20 \mathrm{E}-11$ & $1.44 \mathrm{E}-12$ & $4.78 \mathrm{E}-13$ & $1.42 \mathrm{E}-12$ & $4.46 \mathrm{E}-12$ & $1.45 \mathrm{E}-12$ & $4.25 \mathrm{E}-12$ & $3.83 \mathrm{E}-12$ & $7.02 \mathrm{E}-12$ \\
\hline 3.8 & $1.96 \mathrm{E}-11$ & $1.28 \mathrm{E}-12$ & $4.26 \mathrm{E}-13$ & $1.26 \mathrm{E}-12$ & $3.97 \mathrm{E}-12$ & $1.29 \mathrm{E}-12$ & $3.79 \mathrm{E}-12$ & $3.42 \mathrm{E}-12$ & $6.26 \mathrm{E}-12$ \\
\hline 3.9 & $1.75 \mathrm{E}-11$ & $1.14 \mathrm{E}-12$ & $3.79 \mathrm{E}-13$ & $1.12 \mathrm{E}-12$ & $3.54 \mathrm{E}-12$ & $1.15 \mathrm{E}-12$ & 3.37E -12 & $3.04 \mathrm{E}-12$ & $5.58 \mathrm{E}-12$ \\
\hline 4.0 & $1.56 \mathrm{E}-11$ & $1.02 \mathrm{E}-12$ & $3.38 \mathrm{E}-13$ & $1.00 \mathrm{E}-12$ & $3.16 \mathrm{E}-12$ & $1.03 \mathrm{E}-12$ & $3.01 \mathrm{E}-12$ & $2.71 \mathrm{E}-12$ & $4.97 \mathrm{E}-12$ \\
\hline 4.1 & $1.39 \mathrm{E}-11$ & $9.07 \mathrm{E}-13$ & $3.01 \mathrm{E}-13$ & $8.93 \mathrm{E}-13$ & $2.81 \mathrm{E}-12$ & $9.14 \mathrm{E}-13$ & $2.68 \mathrm{E}-12$ & $2.42 \mathrm{E}-12$ & $4.43 \mathrm{E}-12$ \\
\hline 4.2 & $1.24 \mathrm{E}-11$ & $8.09 \mathrm{E}-13$ & $2.69 \mathrm{E}-13$ & $7.95 \mathrm{E}-13$ & $2.51 \mathrm{E}-12$ & $8.14 \mathrm{E}-13$ & $2.39 \mathrm{E}-12$ & $2.15 \mathrm{E}-12$ & $3.94 \mathrm{E}-12$ \\
\hline 4.3 & $1.10 \mathrm{E} \quad 11$ & $7.21 \mathrm{E} \quad 13$ & $2.39 \mathrm{E} \quad 13$ & $7.08 \mathrm{E}-13$ & $2.23 \mathrm{E} \quad 12$ & $7.26 \mathrm{E}-13$ & $2.13 \mathrm{E}-12$ & $1.92 \mathrm{E} \quad 12$ & $3.51 \mathrm{E} \quad 12$ \\
\hline 4.4 & $9.82 \mathrm{E}-12$ & $6.42 \mathrm{E}-13$ & $2.13 \mathrm{E}-13$ & $6.31 \mathrm{E}-13$ & $1.99 \mathrm{E}-12$ & $6.47 \mathrm{E}-13$ & $1.89 \mathrm{E}-12$ & $1.71 \mathrm{E}-12$ & $3.13 \mathrm{E}-12$ \\
\hline 4.5 & $8.75 \mathrm{E}-12$ & $5.72 \mathrm{E}-13$ & $1.90 \mathrm{E}-13$ & $5.62 \mathrm{E}-13$ & $1.78 \mathrm{E}-12$ & $5.77 \mathrm{E}-13$ & $1.69 \mathrm{E}-12$ & $1.52 \mathrm{E} \quad 12$ & $2.78 \mathrm{E}-12$ \\
\hline 4.6 & $7.80 \mathrm{E}-12$ & $5.10 \mathrm{E}-13$ & $1.69 \mathrm{E}-13$ & $5.00 \mathrm{E}-13$ & $1.58 \mathrm{E}-12$ & $5.14 \mathrm{E}-13$ & $1.50 \mathrm{E}-12$ & $1.35 \mathrm{E}-12$ & $2.48 \mathrm{E}-12$ \\
\hline 4.7 & $6.95 \mathrm{E}-12$ & $4.55 \mathrm{E}-13$ & $1.51 \mathrm{E}-13$ & $4.45 \mathrm{E} \quad 13$ & $1.41 \mathrm{E}-12$ & $4.58 \mathrm{E}-13$ & $1.33 \mathrm{E}-12$ & $1.20 \mathrm{E}-12$ & $2.21 \mathrm{E}-12$ \\
\hline 4.8 & $6.19 \mathrm{E}-12$ & $4.05 \mathrm{E}-13$ & $1.35 \mathrm{E}-13$ & $3.96 \mathrm{E}-13$ & $1.26 \mathrm{E}-12$ & $4.08 \mathrm{E}-13$ & $1.19 \mathrm{E}-12$ & $1.07 \mathrm{E}-12$ & $1.96 \mathrm{E}-12$ \\
\hline 4.9 & $5.52 \mathrm{E}-12$ & $3.61 \mathrm{E}-13$ & $1.20 \mathrm{E}-13$ & $3.52 \mathrm{E}-13$ & $1.12 \mathrm{E}-12$ & $3.64 \mathrm{E}-13$ & $1.06 \mathrm{E}-12$ & $9.52 \mathrm{E}-13$ & $1.74 \mathrm{E}-12$ \\
\hline 5.0 & $4.91 \mathrm{E}-12$ & $3.22 \mathrm{E}-13$ & $1.07 \mathrm{E}-13$ & $3.13 \mathrm{E}-13$ & $9.98 \mathrm{E}-13$ & $3.24 \mathrm{E}-13$ & $9.38 \mathrm{E}-13$ & $8.45 \mathrm{E}-13$ & $1.55 \mathrm{E}-12$ \\
\hline 5.1 & $4.38 \mathrm{E}-12$ & $2.87 \mathrm{E}-13$ & $9.53 \mathrm{E}-14$ & $2.78 \mathrm{E}-13$ & $8.90 \mathrm{E}-13$ & $2.89 \mathrm{E}-13$ & $8.33 \mathrm{E}-13$ & $7.50 \mathrm{E}-13$ & $1.38 \mathrm{E}-12$ \\
\hline 5.2 & $3.90 \mathrm{E}-12$ & $2.55 \mathrm{E}-13$ & $8.49 \mathrm{E}-14$ & $2.46 \mathrm{E}-13$ & $7.93 \mathrm{E}-13$ & $2.58 \mathrm{E}-13$ & $7.39 \mathrm{E} \quad 13$ & $6.65 \mathrm{E}-13$ & $1.22 \mathrm{E}-12$ \\
\hline 5.3 & $3.47 \mathrm{E}-12$ & $2.28 \mathrm{E}-13$ & 7.57E -14 & $2.18 \mathrm{E}-13$ & $7.06 \mathrm{E}-13$ & $2.30 \mathrm{E}-13$ & $6.55 \mathrm{E}-13$ & $5.89 \mathrm{E}-13$ & $1.08 \mathrm{E}-12$ \\
\hline 5.4 & $3.09 \mathrm{E}-12$ & $2.03 \mathrm{E}-13$ & $6.74 \mathrm{E}-14$ & $1.93 \mathrm{E}-13$ & $6.29 \mathrm{E}-13$ & $2.05 \mathrm{E}-13$ & $5.79 \mathrm{E}-13$ & $5.21 \mathrm{E}-13$ & $9.58 \mathrm{E}-13$ \\
\hline 5.5 & $2.75 \mathrm{E}-12$ & $1.81 \mathrm{E}-13$ & $6.01 \mathrm{E}-14$ & $1.71 \mathrm{E}-13$ & $5.61 \mathrm{E}-13$ & $1.82 \mathrm{E}-13$ & $5.12 \mathrm{E}-13$ & $4.60 \mathrm{E}-13$ & $8.46 \mathrm{E}-13$ \\
\hline 5.6 & $2.45 \mathrm{E}-12$ & $1.61 \mathrm{E}-13$ & $5.35 \mathrm{E}-14$ & $1.50 \mathrm{E}-13$ & $5.00 \mathrm{E}-13$ & $1.62 \mathrm{E}-13$ & $4.51 \mathrm{E}-13$ & $4.05 \mathrm{E}-13$ & $7.46 \mathrm{E}-13$ \\
\hline 5.7 & $2.18 \mathrm{E}-12$ & $1.43 \mathrm{E} \quad 13$ & $4.76 \mathrm{E}-14$ & $1.32 \mathrm{E}-13$ & $4.45 \mathrm{E}-13$ & $1.45 \mathrm{E}-13$ & $3.97 \mathrm{E}-13$ & $3.56 \mathrm{E}-13$ & $6.56 \mathrm{E}-13$ \\
\hline 5.8 & $1.94 \mathrm{E}-12$ & $1.27 \mathrm{E}-13$ & $4.24 \mathrm{E}-14$ & $1.16 \mathrm{E}-13$ & $3.96 \mathrm{E}-13$ & $1.29 \mathrm{E}-13$ & $3.48 \mathrm{E}-13$ & $3.11 \mathrm{E}-13$ & $5.75 \mathrm{E}-13$ \\
\hline 5.9 & $1.72 \mathrm{E}-12$ & $1.13 \mathrm{E}-13$ & $3.77 \mathrm{E}-14$ & $1.01 \mathrm{E}-13$ & $3.53 \mathrm{E}-13$ & $1.15 \mathrm{E}-13$ & $3.04 \mathrm{E}-13$ & $2.72 \mathrm{E}-13$ & $5.02 \mathrm{E}-13$ \\
\hline 6.0 & $1.53 \mathrm{E}-12$ & $1.01 \mathrm{E}-13$ & $3.35 \mathrm{E}-14$ & $8.82 \mathrm{E}-14$ & $3.14 \mathrm{E}-13$ & $1.02 \mathrm{E}-13$ & $2.64 \mathrm{E}-13$ & $2.36 \mathrm{E}-13$ & $4.37 \mathrm{E}-13$ \\
\hline 6.1 & $1.36 \mathrm{E}-12$ & $8.94 \mathrm{E}-14$ & $2.97 \mathrm{E}-14$ & $7.64 \mathrm{E}-14$ & $2.79 \mathrm{E}-13$ & $9.04 \mathrm{E}-14$ & $2.29 \mathrm{E}-13$ & $2.04 \mathrm{E}-13$ & $3.78 \mathrm{E}-13$ \\
\hline 6.2 & $1.20 \mathrm{E} \quad 12$ & $7.94 \mathrm{E}-14$ & 2.63E 14 & $6.58 \mathrm{E}-14$ & $2.48 \mathrm{E}-13$ & $8.01 \mathrm{E}-14$ & $1.97 \mathrm{E}-13$ & $1.75 \mathrm{E}-13$ & $3.26 \mathrm{E}-13$ \\
\hline 6.3 & $1.06 \mathrm{E}-12$ & $7.08 \mathrm{E}-14$ & $2.33 \mathrm{E}-14$ & $5.64 \mathrm{E}-14$ & $2.20 \mathrm{E}-13$ & $7.10 \mathrm{E}-14$ & $1.69 \mathrm{E}-13$ & $1.51 \mathrm{E}-13$ & $2.79 \mathrm{E}-13$ \\
\hline 6.4 & $9.38 \mathrm{E}-13$ & $6.46 \mathrm{E}-14$ & $2.07 \mathrm{E}-14$ & $4.80 \mathrm{E}-14$ & $1.97 \mathrm{E}-13$ & $6.32 \mathrm{E}-14$ & $1.44 \mathrm{E}-13$ & $1.35 \mathrm{E}-13$ & $2.38 \mathrm{E}-13$ \\
\hline 6.5 & $8.27 \mathrm{E}-13$ & $6.19 \mathrm{E}-14$ & $1.90 \mathrm{E}-14$ & $4.05 \mathrm{E}-14$ & $1.80 \mathrm{E}-13$ & $5.72 \mathrm{E}-14$ & $1.22 \mathrm{E}-13$ & $1.30 \mathrm{E}-13$ & $2.03 \mathrm{E}-13$ \\
\hline 6.6 & 7.27E $\quad 13$ & $6.33 \mathrm{E}-14$ & $1.84 \mathrm{E}-14$ & $3.40 \mathrm{E}-14$ & $1.68 \mathrm{E} \quad 13$ & $5.39 \mathrm{E}-14$ & $1.04 \mathrm{E}-13$ & $1.39 \mathrm{E} \quad 13$ & $1.73 \mathrm{E}-13$ \\
\hline 6.7 & $6.37 \mathrm{E}-13$ & $6.78 \mathrm{E}-14$ & $1.92 \mathrm{E}-14$ & $2.84 \mathrm{E}-14$ & $1.61 \mathrm{E}-13$ & $5.34 \mathrm{E}-14$ & $8.94 \mathrm{E} \quad 14$ & $1.57 \mathrm{E}-13$ & $1.49 \mathrm{E}-13$ \\
\hline 6.8 & $5.56 \mathrm{E}-13$ & $7.31 \mathrm{E}-14$ & $2.08 \mathrm{E}-14$ & $2.36 \mathrm{E}-14$ & $1.56 \mathrm{E}-13$ & $5.48 \mathrm{E}-14$ & $7.71 \mathrm{E}-14$ & $1.78 \mathrm{E}-13$ & $1.28 \mathrm{E}-13$ \\
\hline 6.9 & $4.83 \mathrm{E} \quad 13$ & $7.67 \mathrm{E}-14$ & $2.25 \mathrm{E}-14$ & $1.95 \mathrm{E}-14$ & $1.50 \mathrm{E}-13$ & $5.65 \mathrm{E}-14$ & $6.67 \mathrm{E}-14$ & $1.92 \mathrm{E}-13$ & $1.11 \mathrm{E}-13$ \\
\hline 7.0 & $4.18 \mathrm{E}-13$ & $7.69 \mathrm{E}-14$ & $2.35 \mathrm{E}-14$ & $1.60 \mathrm{E} \quad 14$ & $1.40 \mathrm{E} \quad 13$ & $5.69 \mathrm{E}-14$ & $5.74 \mathrm{E} \quad 14$ & $1.96 \mathrm{E}-13$ & $9.50 \mathrm{E}-14$ \\
\hline 7.1 & $3.59 \mathrm{E}-13$ & $7.34 \mathrm{E}-14$ & $2.33 \mathrm{E}-14$ & $1.30 \mathrm{E}-14$ & $1.28 \mathrm{E}-13$ & $5.50 \mathrm{E}-14$ & $4.89 \mathrm{E}-14$ & $1.89 \mathrm{E}-13$ & $8.04 \mathrm{E}-14$ \\
\hline
\end{tabular}


Table 4 (continued)

\begin{tabular}{|c|c|c|c|c|c|c|c|c|c|}
\hline \multirow[t]{2}{*}{$\log _{10} T(\mathrm{~K})$} & \multicolumn{9}{|c|}{$\alpha_{\mathrm{R}}\left(\mathrm{cm}^{3} \mathrm{~s}^{-1}\right)$} \\
\hline & $1 \mathrm{~s}^{21} \mathrm{~S}$ & $1 \mathrm{~s} 2 \mathrm{~s}^{1} \mathrm{~S}_{0}$ & $1 \mathrm{~s}^{3} \mathrm{~s}^{1} \mathrm{~S}_{0}$ & $1 \mathrm{~s} 2 \mathrm{p}^{3} \mathrm{P}_{0}^{0}$ & $1 s 2 s^{3} s_{1}$ & $1 \mathrm{~s} 3 \mathrm{~s}^{3} \mathrm{~S}_{1}$ & $1 \mathrm{~s} 2 \mathrm{p}^{3} \mathrm{P}_{1}^{0}$ & $1 \mathrm{~s} 2 \mathrm{p}^{1} \mathrm{P}_{1}^{0}$ & $1 s 2 \mathrm{p}^{3} \mathrm{P}_{2}^{0}$ \\
\hline & \multicolumn{9}{|l|}{ Si XIII } \\
\hline BE: & -179.0 & 42.9 & -19.0 & 43.0 & 44.0 & -19.3 & 42.9 & 42.1 & 42.8 \\
\hline 7.2 & $3.07 \mathrm{E}-13$ & $6.68 \mathrm{E}-14$ & $2.19 \mathrm{E}-14$ & $1.05 \mathrm{E}-14$ & $1.13 \mathrm{E}-13$ & $5.09 \mathrm{E}-14$ & $4.10 \mathrm{E}-14$ & $1.71 \mathrm{E}-13$ & $6.70 \mathrm{E}-14$ \\
\hline 7.3 & $2.60 \mathrm{E}-13$ & $5.83 \mathrm{E}-14$ & $1.97 \mathrm{E}-14$ & $8.37 \mathrm{E}-15$ & $9.71 \mathrm{E}-14$ & $4.52 \mathrm{E}-14$ & $3.38 \mathrm{E}-14$ & $1.49 \mathrm{E}-13$ & $5.48 \mathrm{E}-14$ \\
\hline 7.4 & $2.19 \mathrm{E}-13$ & $4.92 \mathrm{E}-14$ & $1.70 \mathrm{E}-14$ & $6.62 \mathrm{E}-15$ & $8.13 \mathrm{E}-14$ & $3.87 \mathrm{E}-14$ & $2.74 \mathrm{E}-14$ & $1.24 \mathrm{E}-13$ & $4.41 \mathrm{E}-14$ \\
\hline 7.5 & $1.83 \mathrm{E}-13$ & $4.03 \mathrm{E}-14$ & $1.41 \mathrm{E}-14$ & $5.19 \mathrm{E}-15$ & $6.67 \mathrm{E}-14$ & $3.22 \mathrm{E}-14$ & $2.18 \mathrm{E}-14$ & $1.01 \mathrm{E}-13$ & $3.50 \mathrm{E}-14$ \\
\hline 7.6 & $1.51 \mathrm{E}-13$ & $3.22 \mathrm{E}-14$ & $1.14 \mathrm{E}-14$ & $4.03 \mathrm{E}-15$ & $5.37 \mathrm{E}-14$ & $2.60 \mathrm{E}-14$ & $1.71 \mathrm{E}-14$ & $7.93 \mathrm{E}-14$ & $2.74 \mathrm{E}-14$ \\
\hline 7.7 & $1.24 \mathrm{E}-13$ & $2.53 \mathrm{E}-14$ & $9.05 \mathrm{E}-15$ & $3.11 \mathrm{E}-15$ & $4.26 \mathrm{E}-14$ & $2.06 \mathrm{E}-14$ & $1.32 \mathrm{E} \quad 14$ & $6.13 \mathrm{E}-14$ & $2.11 \mathrm{E}-14$ \\
\hline 7.8 & $1.01 \mathrm{E}-13$ & $1.96 \mathrm{E}-14$ & $7.03 \mathrm{E}-15$ & $2.39 \mathrm{E}-15$ & $3.33 \mathrm{E}-14$ & $1.61 \mathrm{E}-14$ & $1.02 \mathrm{E}-14$ & $4.66 \mathrm{E}-14$ & $1.62 \mathrm{E}-14$ \\
\hline 7.9 & $8.20 \mathrm{E}-14$ & $1.50 \mathrm{E}-14$ & $5.39 \mathrm{E}-15$ & $1.82 \mathrm{E}-15$ & $2.59 \mathrm{E}-14$ & $1.24 \mathrm{E}-14$ & $7.72 \mathrm{E}-15$ & $3.50 \mathrm{E}-14$ & $1.23 \mathrm{E}-14$ \\
\hline 8.0 & $6.59 \mathrm{E} \quad 14$ & $1.13 \mathrm{E}-14$ & $4.08 \mathrm{E}-15$ & $1.38 \mathrm{E}-15$ & $1.99 \mathrm{E}-14$ & $9.40 \mathrm{E}-15$ & $5.83 \mathrm{E}-15$ & $2.60 \mathrm{E}-14$ & $9.27 \mathrm{E}-15$ \\
\hline 8.1 & $5.25 \mathrm{E}-14$ & $8.53 \mathrm{E}-15$ & $3.06 \mathrm{E}-15$ & $1.05 \mathrm{E}-15$ & $1.52 \mathrm{E}-14$ & $7.09 \mathrm{E}-15$ & $4.38 \mathrm{E}-15$ & $1.91 \mathrm{E}-14$ & $6.95 \mathrm{E}-15$ \\
\hline 8.2 & $4.16 \mathrm{E}-14$ & $6.37 \mathrm{E}-15$ & $2.28 \mathrm{E}-15$ & $7.89 \mathrm{E}-16$ & $1.16 \mathrm{E}-14$ & $5.31 \mathrm{E}-15$ & $3.27 \mathrm{E}-15$ & $1.40 \mathrm{E}-14$ & $5.19 \mathrm{E}-15$ \\
\hline 8.3 & $3.27 \mathrm{E}-14$ & $4.73 \mathrm{E}-15$ & $1.69 \mathrm{E}-15$ & $5.92 \mathrm{E}-16$ & $8.73 \mathrm{E}-15$ & $3.96 \mathrm{E}-15$ & $2.43 \mathrm{E}-15$ & $1.02 \mathrm{E}-14$ & $3.86 \mathrm{E}-15$ \\
\hline 8.4 & $2.56 \mathrm{E}-14$ & $3.50 \mathrm{E}-15$ & $1.25 \mathrm{E}-15$ & $4.43 \mathrm{E}-16$ & $6.57 \mathrm{E}-15$ & $2.93 \mathrm{E}-15$ & $1.80 \mathrm{E}-15$ & $7.38 \mathrm{E}-15$ & $2.87 \mathrm{E}-15$ \\
\hline 8.5 & $1.99 \mathrm{E}-14$ & $2.59 \mathrm{E}-15$ & $9.15 \mathrm{E}-16$ & $3.31 \mathrm{E}-16$ & $4.93 \mathrm{E}-15$ & $2.17 \mathrm{E}-15$ & $1.33 \mathrm{E}-15$ & $5.34 \mathrm{E}-15$ & $2.12 \mathrm{E}-15$ \\
\hline 8.6 & $1.54 \mathrm{E}-14$ & $1.90 \mathrm{E}-15$ & $6.70 \mathrm{E}-16$ & $2.46 \mathrm{E}-16$ & $3.69 \mathrm{E}-15$ & $1.60 \mathrm{E}-15$ & $9.82 \mathrm{E}-16$ & $3.85 \mathrm{E}-15$ & $1.56 \mathrm{E}-15$ \\
\hline 8.7 & $1.18 \mathrm{E}-14$ & $1.40 \mathrm{E}-15$ & $4.89 \mathrm{E}-16$ & $1.83 \mathrm{E}-16$ & $2.75 \mathrm{E}-15$ & $1.17 \mathrm{E}-15$ & $7.23 \mathrm{E}-16$ & $2.77 \mathrm{E}-15$ & $1.15 \mathrm{E}-15$ \\
\hline 8.8 & $9.07 \mathrm{E}-15$ & $1.02 \mathrm{E}-15$ & $3.57 \mathrm{E}-16$ & $1.36 \mathrm{E}-16$ & $2.04 \mathrm{E}-15$ & $8.60 \mathrm{E}-16$ & $5.31 \mathrm{E}-16$ & $1.99 \mathrm{E}-15$ & $8.47 \mathrm{E}-16$ \\
\hline 8.9 & $6.92 \mathrm{E}-15$ & $7.50 \mathrm{E}-16$ & $2.60 \mathrm{E}-16$ & $1.01 \mathrm{E}-16$ & $1.52 \mathrm{E}-15$ & $6.29 \mathrm{E}-16$ & $3.90 \mathrm{E}-16$ & $1.42 \mathrm{E}-15$ & $6.22 \mathrm{E}-16$ \\
\hline 9.0 & $5.26 \mathrm{E}-15$ & $5.48 \mathrm{E}-16$ & $1.89 \mathrm{E}-16$ & $7.43 \mathrm{E}-17$ & $1.12 \mathrm{E}-15$ & $4.60 \mathrm{E}-16$ & $2.85 \mathrm{E}-16$ & $1.02 \mathrm{E}-15$ & $4.56 \mathrm{E}-16$ \\
\hline
\end{tabular}

$\mathrm{BE}$ is the binding energy of the level in Rydberg. 


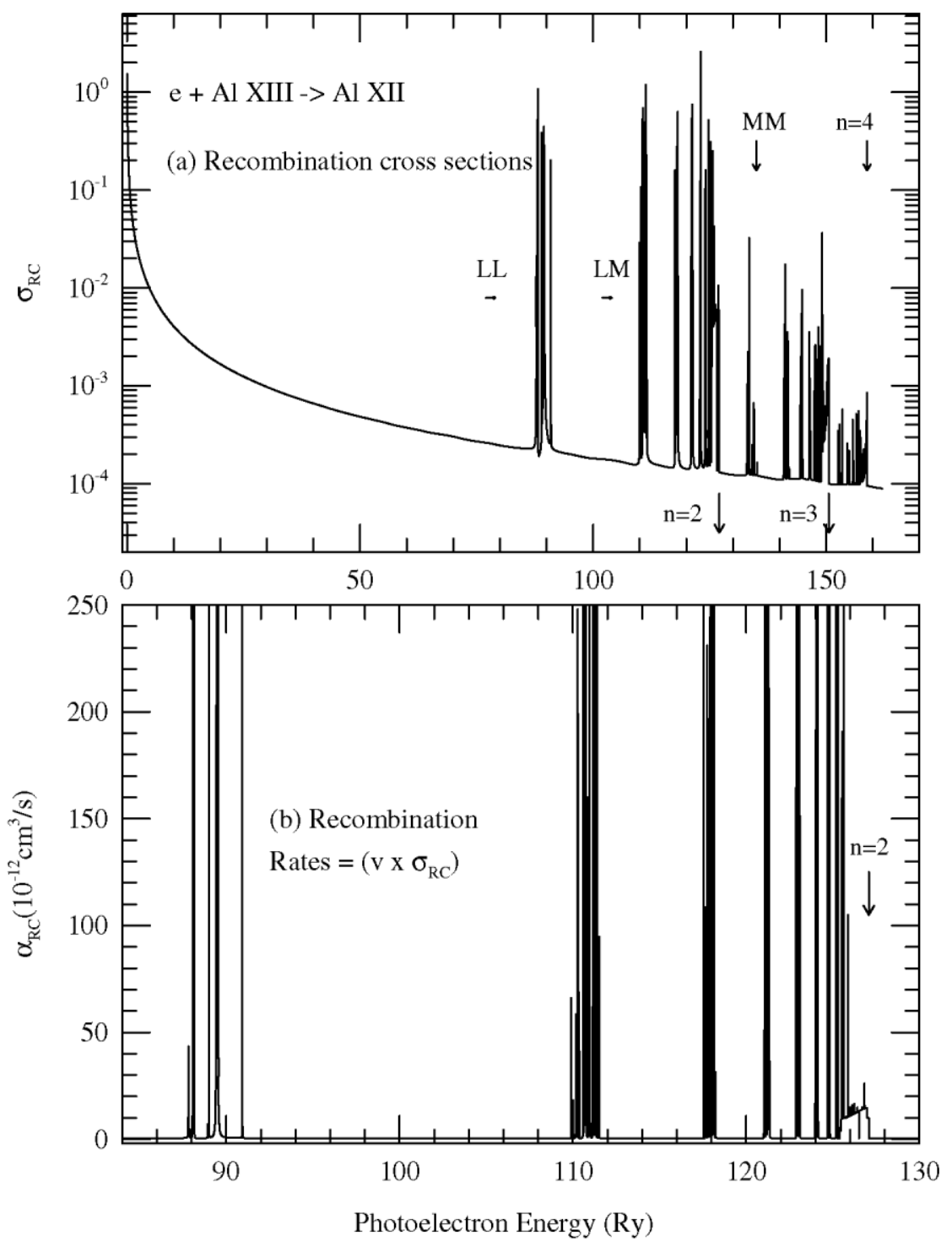

Fig. 10. (a) Total unified ( + ion) recombination cross-sections, $\sigma_{R C}$ and (b) unified recombination rate coefficients, $\alpha_{\mathrm{RC}}(\mathrm{E})$ versus photoelectron energy for (e + Al XIII ? Al XII). The separated resonance complexes, LL, LM, etc., of $n$ $=2$ and MM, MN, etc., of $n=3$ and those of $n=4$ thresholds can be noted in (a). $\alpha_{\mathrm{RC}}(\mathrm{E})$ is a measurable quantity. 


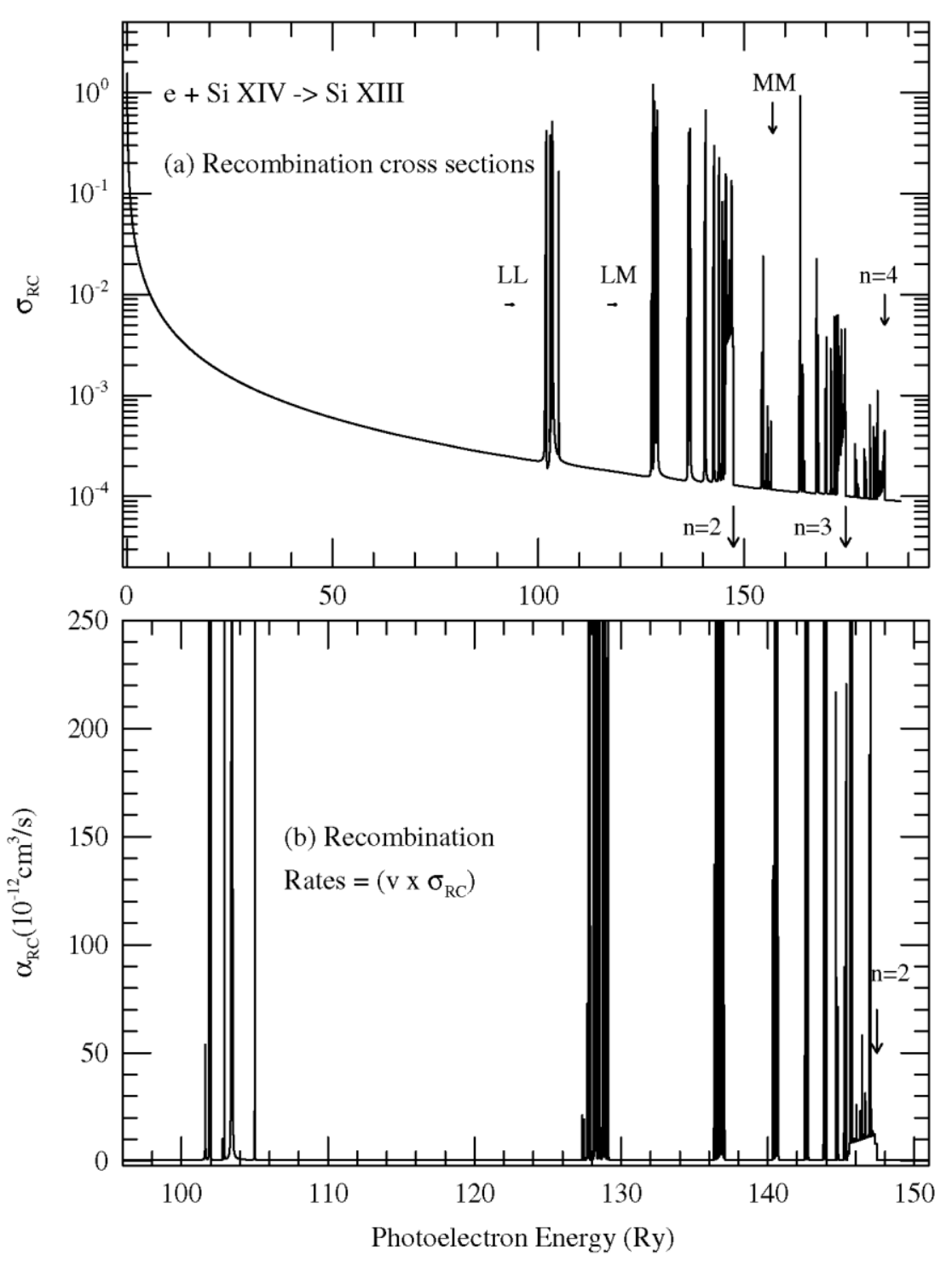

Fig. 11. (a) Total unified (e + ion) recombination cross-sections, $\sigma_{R C}$ and (b) unified recombination rate coefficients, $\alpha_{\mathrm{RC}}(\mathrm{E})$ versus photoelectron energy for (e + Si XIV ? Si XIII). The separated resonance complexes, LL, LM, etc., of $n$ $=2$ and $M M, M N$, etc., of $n=3$ and those of $n=4$ thresholds can be noted in (a). $\alpha_{R C}(E)$ is a measurable quantity.

Similar to the excited levels of the Li-like ions (Fig. 3 for Al XI), all excited levels of the He-like Al XII and Si XIII show existence of PEC resonances in $\sigma_{\mathrm{PI}}$. Fig. 9 presents level specific total $\sigma_{\mathrm{PI}}$ of the excited Rydberg series of levels, 1 sns $\left({ }^{1} \mathrm{~S}_{0}\right)$ with $2 \leq \mathrm{n} \leq 7$ for Si XIII. The PEC resonances can be seen at energies (pointed by arrows) about 147 Ry due to core excitations to $\mathrm{n}=$ 2 levels, $2 \mathrm{p}^{2} \mathrm{P}_{1 / 2,3 / 2}^{\mathrm{o}}$, about $175 \mathrm{Ry}$ due to $\mathrm{n}=3$ levels $3 \mathrm{p}^{2} \mathrm{P}_{1 / 2,3 / 2}^{\mathrm{o}}$, and at about 184 Ry due to $\mathrm{n}=4$ levels $4 \mathrm{p}^{2} \mathrm{P}_{12,3 / 2}^{\mathrm{o}}$ transitions. The background enhancement due to PEC are distinct from that of narrow Rydberg autoionizing resonances.

Partial photoionization cross-sections for Al XII and Si XIII are similar to those of the total, except they have less resonances and lower background.

\subsubsection{Electron-ion recombination}


Level-specific recombination rate coefficients $\alpha_{R}(\mathrm{nSLJ})$ are presented for all 190 fine structure levels of Al XII and 189 levels of Si XIII with $0 \leq J \leq 10$ and $n \leq 10$. Table 4 presents a sample set of level specific recombination rates for the ground level $1 \mathrm{~s}^{2}\left({ }^{1} \mathrm{~S}_{0}\right)$, and excited levels $1 \mathrm{~s} 2 \mathrm{~s}\left({ }^{1} \mathrm{~S}_{0}\right), 1 \mathrm{~s} 2 \mathrm{~s}\left({ }^{3} \mathrm{~S}_{1}\right), 1 \mathrm{~s} 2 \mathrm{p}\left({ }^{1} \mathrm{P}_{1}^{\mathrm{o}}\right), 1 \mathrm{~s} 2 \mathrm{p}\left({ }^{3} \mathrm{P}_{0}^{0}\right) 1 \mathrm{~s} 2 \mathrm{p}\left({ }^{3} \mathrm{P}_{1}^{\mathrm{o}}\right), 1 \mathrm{~s} 2 \mathrm{p}\left({ }^{3} \mathrm{P}_{2}^{\mathrm{o}}\right), 1 \mathrm{~s} 3 \mathrm{~s}\left({ }^{1} \mathrm{~S}_{0}\right)$, and $1 \mathrm{~s} 3 \mathrm{~s}\left({ }^{3} \mathrm{~S}_{1}\right)$. The set includes the levels corresponding to the X-ray $w, x, y$, and $z$ lines of Al XII and Si XIII. The rates show a relatively smooth decay with temperature and a small shoulder at high temperature due to DR resonances.

Both the total electron-ion recombination collision strength $(\sigma R C)$ and total recombination cross-sections $\left(\sigma_{\mathrm{R}} \mathrm{C}\right)$ are obtained for the recombined He-like ions Al XII and Si XIII. Both quantities have similar features. Only unified $\sigma_{\mathrm{RC}}$ is presented in the top panel of Fig. 10 for Al XII and of Fig. 11 for Si XIII. The unified $\sigma_{\mathrm{RC}}$ diverges at zero photoelectron energy, but decays smoothly with energy until resonance complexes appear at very high energies. The resonance complexes LL (2121'), LM (2131'), etc., converging on to the $\mathrm{n}=2$ thresholds, MM, MN, etc., converging on to the $n=3$ thresholds, and NN, etc., converging on to the $n=4$ thresholds. LL LM complexes can be observed in the recombination spectra as satellite lines. The resonances become much weaker by orders of magnitude and narrower beyond $n=2$ thresholds.

The lower panels of Figs. 10 and 11 present the velocity dependent photorecombination rates $\alpha_{R C}(E)$ for Al XII and Si XIII, respectively. The high-peak resonant part up to $n=2$ of $\alpha_{R C}(E)$ is presented as these resonant features can be observed in experiment. 
Table 5

Total recombination rate coefficients $\alpha_{\mathrm{R}}(T)$ for Al XI, Al XII, Al XIII, Si XII, Si XIII, Si XIV

\begin{tabular}{|c|c|c|c|c|c|c|}
\hline \multirow[t]{2}{*}{$\log _{10} T(\mathrm{~K})$} & \multicolumn{6}{|c|}{$\alpha_{R}\left(\mathrm{~cm}^{3} \mathrm{~s}^{-1}\right)$} \\
\hline & $\mathrm{Al} \mathrm{XI}$ & Al XII & Al XIII & Si XII & Si XIII & Si XIV \\
\hline 1.0 & $5.00 \mathrm{E}-09$ & 6.33F 09 & $7.87 \mathrm{E}-09$ & $5.97 \mathrm{E}-09$ & $7.46 \mathrm{E}-09$ & $9.16 \mathrm{E} \quad 09$ \\
\hline 1.1 & 4.42F -09 & $5.61 \mathrm{E} \quad 09$ & $6.98 \mathrm{E} \quad 09$ & 5.29E 09 & $6.62 \mathrm{E}-09$ & 8.12E 09 \\
\hline 1.2 & $3.91 \mathrm{E}-09$ & 4.97F -09 & 6.19E 09 & $4.68 \mathrm{E} \quad 09$ & 5.87E 09 & $7.20 \mathrm{E} \quad 09$ \\
\hline 1.3 & 3.45E 09 & 4.39F 09 & $5.48 \mathrm{E} \quad 09$ & 4.13E 09 & 5.19E 09 & 6.39E 09 \\
\hline 1.4 & $3.05 \mathrm{E} \quad 09$ & $3.88 \mathrm{E} \quad 09$ & 4.84E 09 & 3.65E 09 & $4.58 \mathrm{E} \quad 09$ & $5.65 \mathrm{E} \quad 09$ \\
\hline 1.5 & 2.69E 09 & 3.43E 09 & $4.28 \mathrm{E}-09$ & 3.22E 09 & $4.05 \mathrm{E} \quad 09$ & 4.99E 09 \\
\hline 1.6 & 2.37E 09 & $3.02 \mathrm{E} \quad 09$ & $3.78 \mathrm{E}-09$ & $2.84 \mathrm{E} \quad 09$ & $3.58 \mathrm{E} \quad 09$ & 4.41E 09 \\
\hline 1.7 & 2.08E 09 & 2.66E 09 & 3.33E 09 & 2.50E $\quad 09$ & $3.15 \mathrm{E}-09$ & 3.89E 09 \\
\hline 1.8 & 1.83E 09 & 2.35E 09 & 2.94E 09 & $2.20 \mathrm{E} \quad 09$ & $2.78 \mathrm{E}-09$ & $3.43 \mathrm{E}-09$ \\
\hline 1.9 & 1.61E 09 & 2.06E 09 & 2.59E 09 & $1.93 \mathrm{E} 09$ & 2.45E 09 & $3.03 \mathrm{E} \quad 09$ \\
\hline 2.0 & 1.41E 09 & $1.81 \mathrm{E} \quad 09$ & $2.28 \mathrm{E} \quad 09$ & $1.70 \mathrm{E} \quad 09$ & 2.15E 09 & 2.67E 09 \\
\hline 2.1 & 1.24F 09 & 1.59E 09 & 2.00E 09 & 1.49E 09 & 1.89E 09 & 2.35E 09 \\
\hline 2.2 & $1.08 \mathrm{E}-09$ & $1.40 \mathrm{E} \quad 09$ & 1.76E 09 & $1.31 \mathrm{E}-09$ & 1.66E 09 & $2.06 \mathrm{E} \quad 09$ \\
\hline 2.3 & $9.48 \mathrm{E}-10$ & $1.22 \mathrm{E} \quad 09$ & 1.54E 09 & 1.14E 09 & $1.45 \mathrm{E} \quad 09$ & $1.81 \mathrm{E} 09$ \\
\hline 2.4 & $8.29 \mathrm{E}-10$ & $1.07 \mathrm{E} \quad 09$ & $1.36 \mathrm{E}-09$ & $1.00 \mathrm{E} \quad 09$ & $1.28 \mathrm{E} \quad 09$ & 1.59E 09 \\
\hline 2.5 & $7.25 \mathrm{E}-10$ & $9.39 \mathrm{E} \quad 10$ & 1.19E 09 & $8.75 \mathrm{E}-10$ & $1.12 \mathrm{E} \quad 09$ & $1.39 \mathrm{E} \quad 09$ \\
\hline 2.6 & $6.33 \mathrm{E}-10$ & $8.21 \mathrm{E}-10$ & $1.04 \mathrm{E} \quad 09$ & $7.64 \mathrm{E}-10$ & $9.77 \mathrm{E}-10$ & $1.22 \mathrm{E} \quad 09$ \\
\hline 2.7 & $5.52 \mathrm{E}-10$ & $7.18 \mathrm{E}-10$ & $9.13 \mathrm{E}-10$ & $6.67 \mathrm{E}-10$ & $8.55 \mathrm{E}-10$ & $1.07 \mathrm{E} \quad 09$ \\
\hline 2.8 & $4.81 \mathrm{E}-10$ & $6.27 \mathrm{E}-10$ & $8.00 \mathrm{E}-10$ & $5.82 \mathrm{E}-10$ & $7.48 \mathrm{E}-10$ & $9.39 \mathrm{E}-10$ \\
\hline 2.9 & 4.19E -10 & $5.48 \mathrm{E}-10$ & $6.99 \mathrm{E}-10$ & $5.07 \mathrm{E}-10$ & $6.53 \mathrm{E}-10$ & $8.20 \mathrm{E}-10$ \\
\hline 3.0 & $3.65 \mathrm{~F}-10$ & $4.78 \mathrm{E} \quad 10$ & $6.11 \mathrm{E}-10$ & $4.42 \mathrm{E}-10$ & $5.70 \mathrm{E}-10$ & $7.18 \mathrm{E}-10$ \\
\hline 3.1 & $3.17 \mathrm{E}-10$ & 4.17E -10 & $5.34 \mathrm{E}-10$ & $3.84 \mathrm{~F}-10$ & $4.97 \mathrm{E}-10$ & $6.27 \mathrm{E}-10$ \\
\hline 3.2 & $2.76 \mathrm{~F}-10$ & 3.63E -10 & $4.66 \mathrm{E}-10$ & 3.34E -10 & $4.33 \mathrm{E}-10$ & $5.48 \mathrm{E}-10$ \\
\hline 3.3 & $2.40 \mathrm{~F}-10$ & $3.16 \mathrm{E}-10$ & $4.07 \mathrm{E}-10$ & $2.91 \mathrm{E}-10$ & $3.78 \mathrm{E}-10$ & $4.78 \mathrm{~F}-10$ \\
\hline 3.4 & $2.08 \mathrm{E}-10$ & $2.75 \mathrm{E}-10$ & $3.54 \mathrm{E}-10$ & $2.52 \mathrm{E}-10$ & $3.29 \mathrm{E}-10$ & $4.17 \mathrm{E}-10$ \\
\hline 3.5 & $1.81 \mathrm{E}-10$ & $2.40 \mathrm{E} \quad 10$ & $3.09 \mathrm{E}-10$ & 2.19E $\quad 10$ & $2.86 \mathrm{E}-10$ & $3.63 \mathrm{E}-10$ \\
\hline 3.6 & $1.56 \mathrm{E}-10$ & $2.08 \mathrm{E} \quad 10$ & $2.70 \mathrm{E}-10$ & $1.90 \mathrm{E}-10$ & $2.49 \mathrm{E} \quad 10$ & $3.18 \mathrm{E}-10$ \\
\hline 3.7 & $1.36 \mathrm{E}-10$ & $1.81 \mathrm{E}-10$ & $2.35 \mathrm{E}-10$ & $1.65 \mathrm{E}-10$ & $2.16 \mathrm{E}-10$ & $2.76 \mathrm{E}-10$ \\
\hline 3.8 & $1.17 \mathrm{E}-10$ & 1.57E 10 & $2.04 \mathrm{E}-10$ & $1.42 \mathrm{E}-10$ & $1.88 \mathrm{E}-10$ & $2.40 \mathrm{E}-10$ \\
\hline 3.9 & $1.01 \mathrm{E}-10$ & $1.36 \mathrm{E}-10$ & $1.78 \mathrm{E}-10$ & $1.23 \mathrm{E}-10$ & $1.63 \mathrm{E}-10$ & $2.09 \mathrm{E}-10$ \\
\hline 4.0 & $8.76 \mathrm{E}-11$ & $1.18 \mathrm{E}-10$ & $1.54 \mathrm{E}-10$ & $1.07 \mathrm{E}-10$ & $1.42 \mathrm{E}-10$ & $1.82 \mathrm{E}-10$ \\
\hline 4.1 & $7.56 \mathrm{~F}-11$ & $1.02 \mathrm{E}-10$ & $1.34 \mathrm{E}-10$ & $9.20 \mathrm{E}-11$ & $1.23 \mathrm{E}-10$ & $1.58 \mathrm{E}-10$ \\
\hline 4.2 & $6.52 \mathrm{E}-11$ & $8.85 \mathrm{E}-11$ & $1.16 \mathrm{E}-10$ & $7.94 \mathrm{E}-11$ & $1.06 \mathrm{E}-10$ & $1.37 \mathrm{E}-10$ \\
\hline 4.3 & $5.61 \mathrm{E}-11$ & $7.66 \mathrm{~F}-11$ & $1.01 \mathrm{E}-10$ & $6.84 \mathrm{E}-11$ & $9.19 \mathrm{E}-11$ & $1.19 \mathrm{E}-10$ \\
\hline 4.4 & 4.83E -11 & 6.62E 11 & 8.75F 11 & $5.89 \mathrm{E}-11$ & $7.94 \mathrm{E}-11$ & $1.03 \mathrm{E}-10$ \\
\hline 4.5 & $4.14 \mathrm{~F}-11$ & $5.72 \mathrm{E} \quad 11$ & 7.59E 11 & $5.07 \mathrm{E} \quad 11$ & $6.87 \mathrm{E}-11$ & $8.95 \mathrm{E}-11$ \\
\hline 4.6 & $3.56 \mathrm{E}-11$ & $4.93 \mathrm{E}-11$ & $6.57 \mathrm{E}-11$ & $4.35 \mathrm{E}-11$ & $5.93 \mathrm{E}-11$ & $7.78 \mathrm{E}-11$ \\
\hline 4.7 & 3.05E-11 & $4.25 \mathrm{E}-11$ & $5.68 \mathrm{E}-11$ & $3.73 \mathrm{E}-11$ & $5.11 \mathrm{E}-11$ & $6.72 \mathrm{E}-11$ \\
\hline 4.8 & 2.61F -11 & $3.66 \mathrm{E} \quad 11$ & 4.91E 11 & 3.20E 11 & $4.41 \mathrm{E}-11$ & $5.81 \mathrm{E}-11$ \\
\hline 4.9 & $2.23 \mathrm{E}-11$ & $3.15 \mathrm{E}-11$ & $4.24 \mathrm{E}-11$ & $2.74 \mathrm{E}-11$ & $3.79 \mathrm{E}-11$ & $5.02 \mathrm{E}-11$ \\
\hline 5.0 & $1.90 \mathrm{~F}-11$ & $2.70 \mathrm{E}-11$ & $3.66 \mathrm{E}-11$ & $2.34 \mathrm{E}-11$ & $3.26 \mathrm{E}-11$ & $4.33 \mathrm{E}-11$ \\
\hline 5.1 & $1.62 \mathrm{E}-11$ & 2.32E- 11 & $3.15 \mathrm{E}-11$ & 1.99E -11 & $2.80 \mathrm{E}-11$ & $3.74 \mathrm{E}-11$ \\
\hline 5.2 & $1.38 \mathrm{E}-11$ & $1.99 \mathrm{~F}-11$ & $2.72 \mathrm{E}-11$ & $1.70 \mathrm{E}-11$ & $2.40 \mathrm{~F}-11$ & $3.22 \mathrm{E}-11$ \\
\hline 5.3 & $1.17 \mathrm{E}-11$ & $1.70 \mathrm{E} \quad 11$ & $2.34 \mathrm{E}-11$ & $1.44 \mathrm{~F}-11$ & $2.06 \mathrm{E}-11$ & $2.77 \mathrm{E}-11$ \\
\hline 5.4 & $9.89 \mathrm{E}-12$ & $1.46 \mathrm{E}-11$ & $2.00 \mathrm{E}-11$ & $1.22 \mathrm{E}-11$ & $1.76 \mathrm{E}-11$ & $2.38 \mathrm{E}-11$ \\
\hline 5.5 & $8.35 \mathrm{E}-12$ & $1.24 \mathrm{E}-11$ & $1.72 \mathrm{E}-11$ & $1.03 \mathrm{E}-11$ & $1.51 \mathrm{E}-11$ & $2.04 \mathrm{E}-11$ \\
\hline 5.6 & $7.03 \mathrm{E}-12$ & $1.06 \mathrm{E}-11$ & $1.48 \mathrm{E}-11$ & $8.72 \mathrm{E}-12$ & $1.29 \mathrm{E}-11$ & $1.76 \mathrm{E}-11$ \\
\hline 5.7 & $5.91 \mathrm{E}-12$ & $9.03 \mathrm{E}-12$ & $1.26 \mathrm{E}-11$ & $7.34 \mathrm{E}-12$ & $1.10 \mathrm{E} \quad 11$ & $1.50 \mathrm{E}-11$ \\
\hline 5.8 & 4.96E -12 & 7.67E $\quad 12$ & $1.08 \mathrm{E}-11$ & $6.17 \mathrm{E}-12$ & $9.32 \mathrm{E}-12$ & 1.29E 11 \\
\hline 5.9 & 4.14E- 12 & $6.51 \mathrm{E}-12$ & $9.21 \mathrm{E}-12$ & $5.17 \mathrm{E}-12$ & $7.92 \mathrm{E}-12$ & $1.10 \mathrm{E}-11$ \\
\hline 6.0 & $3.45 \mathrm{E}-12$ & 5.51E -12 & $7.85 \mathrm{E}-12$ & 4.32E -12 & $6.71 \mathrm{E}-12$ & $9.37 \mathrm{E}-12$ \\
\hline 6.1 & $2.87 \mathrm{E}-12$ & $4.65 \mathrm{E}-12$ & $6.68 \mathrm{E}-12$ & $3.59 \mathrm{E}-12$ & $5.67 \mathrm{E}-12$ & $7.98 \mathrm{E}-12$ \\
\hline 6.2 & $2.38 \mathrm{E}-12$ & $3.93 \mathrm{E}-12$ & $5.67 \mathrm{E}-12$ & $2.98 \mathrm{E}-12$ & $4.79 \mathrm{E}-12$ & $6.78 \mathrm{E}-12$ \\
\hline 6.3 & 1.99E -12 & $3.31 \mathrm{E}-12$ & $4.80 \mathrm{E}-12$ & $2.48 \mathrm{E}-12$ & $4.04 \mathrm{E}-12$ & $5.76 \mathrm{E}-12$ \\
\hline 6.4 & $1.71 \mathrm{E}-12$ & $2.82 \mathrm{E}-12$ & $4.05 \mathrm{E} \quad 12$ & $2.07 \mathrm{E}-12$ & $3.41 \mathrm{E}-12$ & $4.88 \mathrm{E}-12$ \\
\hline 6.5 & $1.56 \mathrm{~F}-12$ & $2.45 \mathrm{E}-12$ & $3.43 \mathrm{E}-12$ & $1.79 \mathrm{E}-12$ & $2.91 \mathrm{E}-12$ & $4.11 \mathrm{E}-12$ \\
\hline 6.6 & $1.54 \mathrm{E}-12$ & $2.22 \mathrm{E} \quad 12$ & $2.88 \mathrm{E} \quad 12$ & 1.64F 12 & $2.54 \mathrm{E} \quad 12$ & $3.47 \mathrm{E}-12$ \\
\hline 6.7 & $1.62 \mathrm{E}-12$ & 2.09E -12 & $2.42 \mathrm{E}-12$ & $1.60 \mathrm{E}-12$ & $2.30 \mathrm{E}-12$ & $2.91 \mathrm{E} \quad 12$ \\
\hline 6.8 & $1.72 \mathrm{E}-12$ & $2.03 \mathrm{E}-12$ & $2.02 \mathrm{E}-12$ & 1.64F-12 & $2.16 \mathrm{E}-12$ & $2.44 \mathrm{E}-12$ \\
\hline 6.9 & $1.78 \mathrm{E}-12$ & $1.97 \mathrm{E}-12$ & $1.68 \mathrm{E}-12$ & $1.68 \mathrm{E}-12$ & $2.06 \mathrm{E}-12$ & $2.04 \mathrm{E}-12$ \\
\hline 7.0 & $1.75 \mathrm{E}-12$ & $1.87 \mathrm{E}-12$ & $1.40 \mathrm{E}-12$ & $1.68 \mathrm{E}-12$ & 1.96E -12 & $1.70 \mathrm{E}-12$ \\
\hline 7.1 & 1.64F- 12 & $1.73 \mathrm{E}-12$ & $1.16 \mathrm{E}-12$ & $1.61 \mathrm{E}-12$ & $1.82 \mathrm{E}-12$ & $1.41 \mathrm{E}-12$ \\
\hline 7.2 & $1.47 \mathrm{E}-12$ & $1.54 \mathrm{E}-12$ & $9.54 \mathrm{E}-13$ & $1.48 \mathrm{E}-12$ & $1.65 \mathrm{E}-12$ & $1.16 \mathrm{E}-12$ \\
\hline 7.3 & $1.26 \mathrm{E}-12$ & $1.33 \mathrm{E}-12$ & $7.83 \mathrm{E}-13$ & $1.30 \mathrm{E}-12$ & $1.44 \mathrm{E}-12$ & $9.58 \mathrm{E}-13$ \\
\hline 7.4 & $1.04 \mathrm{E}-12$ & $1.11 \mathrm{E}-12$ & $6.37 \mathrm{E}-13$ & $1.09 \mathrm{E}-12$ & $1.22 \mathrm{E}-12$ & $7.85 \mathrm{E}-13$ \\
\hline 7.5 & $8.40 \mathrm{E}-13$ & $9.03 \mathrm{E}-13$ & $5.18 \mathrm{E}-13$ & $8.96 \mathrm{E}-13$ & 1.01E 12 & $6.37 \mathrm{E}-13$ \\
\hline 7.6 & $6.60 \mathrm{E}-13$ & $7.20 \mathrm{E}-13$ & $4.18 \mathrm{E}-13$ & 7.15E-13 & $8.17 \mathrm{E}-13$ & $5.18 \mathrm{E}-13$ \\
\hline 7.7 & $5.09 \mathrm{E}-13$ & 5.64E- 13 & $3.35 \mathrm{E}-13$ & $5.59 \mathrm{E}-13$ & $6.48 \mathrm{E}-13$ & $4.16 \mathrm{E}-13$ \\
\hline 7.8 & $3.87 \mathrm{E}-13$ & $4.36 \mathrm{E}-13$ & 2.67E -13 & $4.29 \mathrm{E}-13$ & $5.05 \mathrm{E}-13$ & $3.33 \mathrm{E}-13$ \\
\hline 7.9 & $2.90 \mathrm{E}-13$ & $3.34 \mathrm{E}-13$ & $2.12 \mathrm{E}-13$ & $3.25 \mathrm{E}-13$ & $3.90 \mathrm{E}-13$ & $2.65 \mathrm{E}-13$ \\
\hline 8.0 & $2.16 \mathrm{E}-13$ & $2.53 \mathrm{E}-13$ & $1.67 \mathrm{E}-13$ & $2.43 \mathrm{E}-13$ & $2.98 \mathrm{E}-13$ & $2.10 \mathrm{E}-13$ \\
\hline 8.1 & 1.59E -13 & $1.90 \mathrm{E}-13$ & $1.31 \mathrm{E}-13$ & $1.81 \mathrm{E}-13$ & $2.25 \mathrm{E}-13$ & $1.65 \mathrm{E}-13$ \\
\hline 8.2 & $1.17 \mathrm{E}-13$ & $1.42 \mathrm{E}-13$ & $1.02 \mathrm{~F}-13$ & 1.33E 13 & $1.70 \mathrm{E}-13$ & $1.29 \mathrm{E}-13$ \\
\hline
\end{tabular}


Table 5 (continued)

\begin{tabular}{|c|c|c|c|c|c|c|}
\hline \multirow[t]{2}{*}{$\log _{10} T(\mathrm{~K})$} & \multicolumn{6}{|c|}{$\alpha_{\mathrm{R}}\left(\mathrm{cm}^{3} \mathrm{~s}^{-1}\right)$} \\
\hline & $\mathrm{Al} \mathrm{XI}$ & Al XII & Al XIII & Si XII & Si XIII & Si XIV \\
\hline 8.3 & $8.51 \mathrm{E}-14$ & $1.06 \mathrm{E}-13$ & $7.89 \mathrm{E}-14$ & $9.79 \mathrm{E}-14$ & $1.27 \mathrm{E}-13$ & $1.00 \mathrm{E}-13$ \\
\hline 8.4 & $6.19 \mathrm{E}-14$ & $7.86 \mathrm{E}-14$ & $6.05 \mathrm{E}-14$ & $7.15 \mathrm{E}-14$ & $9.47 \mathrm{E}-14$ & $7.74 \mathrm{E}-14$ \\
\hline 8.5 & $4.48 \mathrm{E}-14$ & $5.82 \mathrm{E}-14$ & $4.64 \mathrm{E}-14$ & $5.20 \mathrm{E}-14$ & $7.04 \mathrm{E}-14$ & $5.92 \mathrm{E}-14$ \\
\hline 8.6 & $3.24 \mathrm{E}-14$ & $4.29 \mathrm{E}-14$ & $3.53 \mathrm{E}-14$ & $3.78 \mathrm{E}-14$ & $5.21 \mathrm{E}-14$ & $4.55 \mathrm{E}-14$ \\
\hline 8.7 & $2.34 \mathrm{E}-14$ & $3.16 \mathrm{E}-14$ & $2.67 \mathrm{E}-14$ & $2.74 \mathrm{E}-14$ & $3.85 \mathrm{E}-14$ & $3.44 \mathrm{~F}-14$ \\
\hline 8.8 & $1.68 \mathrm{E}-14$ & $2.33 \mathrm{E}-14$ & $2.01 \mathrm{E}-14$ & $1.98 \mathrm{E}-14$ & $2.84 \mathrm{E}-14$ & $2.60 \mathrm{E}-14$ \\
\hline 8.9 & $1.21 \mathrm{E}-14$ & $1.71 \mathrm{E}-14$ & $1.51 \mathrm{E}-14$ & $1.43 \mathrm{E}-14$ & $2.09 \mathrm{E}-14$ & $1.96 \mathrm{E}-14$ \\
\hline 9.0 & $8.71 \mathrm{E}-15$ & $1.25 \mathrm{E}-14$ & $1.13 \mathrm{E}-14$ & $1.03 \mathrm{E}-14$ & $1.54 \mathrm{E}-14$ & $1.47 \mathrm{E}-14$ \\
\hline
\end{tabular}

The calculated rates show detailed resonant structures except for a small energy range right before the $n=2$ threshold where they are averaged as discussed in Section 2.

Total unified recombination rate coefficients $\alpha_{\mathrm{R}}(\mathrm{T})$ for Al XII and Si XIII are presented for a wide range of temperature, $1 \leq \log _{10} T \leq 9$, in Table 5. The features are illustrated and compared with the available rates in Fig. 12a and b. The BPRM unified $\alpha_{R}(T)$ (solid curve) for these ions start high at very low temperature due to dominance of RR in to an infinite number of high- $n$ levels, decrease with increasing temperature for a large temperature range since the autoionizing resonances do not appear until at high energy. $\alpha_{R}(T)$ forms a small "bump" at high temperature due to dominant DR process, and follows to a smooth decay due to damping of recombination cross-sections by the exponential of the Max-wellian electron distribution.

The present BPRM total unified recombination rate coefficients, $\alpha_{R}(T)$, for Al XII and Si XIII agree very well with the RR rate coefficients by Verner and Ferland (1996, dash) as expected for a two electron system. 


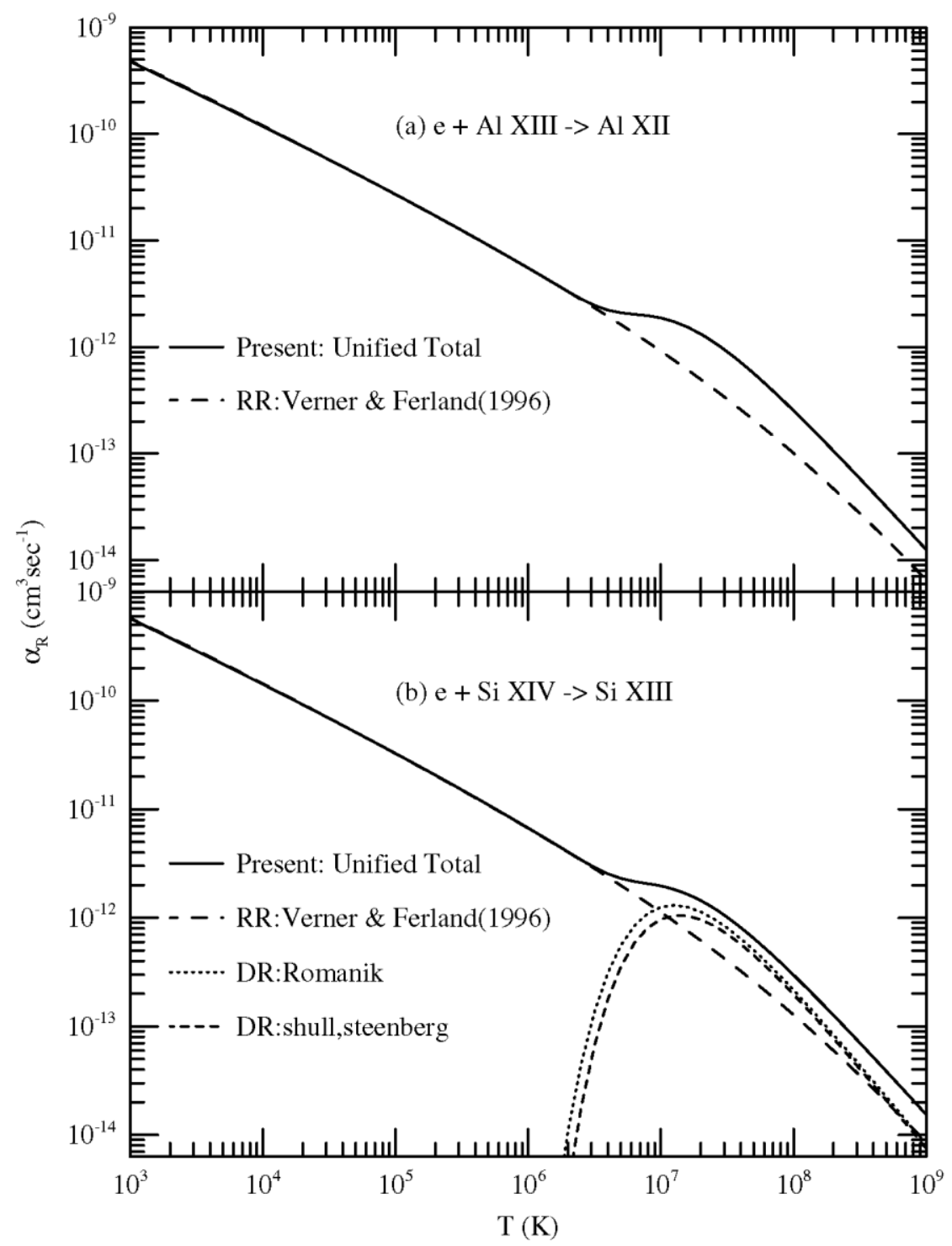

Fig. 12. Total unified recombination rate coefficients $\alpha_{R}(T)$ (solid curves) for (a) Al XII and (b) Si XIII. The RR rates are by Verner and Ferland (1996, dash), the DR rates are by Romanik (1988, dot) and by Shull and van Steenberg (1982, dot and dash).

The high temperature DR rates from isolated resonance approximation by Romanik (dot-dash) and fit by Shull and van Steenberg (1982, short and long dash) for Si XIII agree well with the unified rates although slightly lower. The slight rise in the unified rate can be explained from inclusion of excitation of $n=4$ thresholds in the core and high resolution of resonances in the photoionization cross-sections.

\section{Conclusion}

Extensive sets of results from the unified method employing relativistic BPRM method are presented for the total and level-specific photoionization and recombination cross-sections and rate coefficients for Li-like Al XI, Si XII and He-like Al XII, Si XIII for study of X-ray and UV 
spectroscopy of laboratory and astrophysical sources. The method provides self-consistent atomic parameters for these inverse processes. This report presents the first ab initio relativistic calculations for photoionization and total electron-ion recombination for these ions. The results include (i) level-specific photoionization cross-sections, both partial and total, with inclusion of autoionizing resonances, for levels up to $n=10$, (ii) level-specific recombination rate coefficients, including RR and DR, for all fine structure levels up to $n \leq 10$ and $0 \leq l \leq 9$, (iii) total unified recombination rate coefficients, incorporating both $\mathrm{RR}$ and $\mathrm{DR}$, over a wide temperature range, (iv) total unified recombination cross-sections and velocity averaged recombination rate coefficients as functions of the photoelectron energy for comparison with experiments.

Total recombination rate coefficients have featureless hydro-genic behavior at lower temperature with isoelectronic $z$-depen-dency similar to that of a hydrogenic ion, $z \alpha_{R}\left(H, T / z^{2}\right)$. Hence the rate increases with higher ionic charge. However, the trend does not continue to high temperature where resonances, which do not depend on $z$, appear. The present $\alpha_{\mathrm{RC}}(T)$ agree very well with the combination of existing RR, DR rates.

The present data are expected to be of high accuracy of about $10-15 \%$ based on $(i)$ the very good agreement, less than $1 \%$, of the energies with the experimental values, ( $i$ i) inclusion of important atomic effects such as relativistic effects, radiation damping of resonances, channel couplings, (iii) accuracy of the method from benchmarking with a number of experiments for photoionization (e.g., Nahar, 2004) and electron-ion recombination (e.g., Zhang et al., 1999; Pradhan et al., 2001), and good agreement of the present results with existing rates. The present data are more than sufficient for extrapolation to high $n, \ell$ necessary to account for all recombination-cascade contributions.

All photoionization and recombination data are available electronically from NORAD (NaharOSURadiativeAtomicData) website: www.astronomy.ohio-state.edu/nahar/nahar_radiativeatomicdata.

\section{Acknowledgements}

This work was supported partially by the NASA Astronomy and Physics Research and Analysis program. The computational work was carried out at the Ohio Supercomputer Center in Columbus, OH.

\section{References}

Bell, R.H., Seaton, M.J., 1985. J. Phys. B 18, 1589.

Berrington, K.A., Burke, P.G., Butler, K., Seaton, M.J., Storey, P.J., Taylor, K.T., Yu, Yan, 1987. J. Phys. B 20, 6379.

Berrington, K.A., Eissner, W., Norrington, P.H., 1995. Comput. Phys. Commun. 92, 290.

Canizares et al., 2000. In: Bautista, M.A., Kallman, T.R., Pradhan, A.K. (Eds.), Proceedings of the NASA Workshop on Atomic Data Needs in X-ray Astronomy, 2000, NASA Publications NASA/CP-2000-209968, ADXA. Lhttp://heasarc. gsfc.nasa.gov/docs/heasarc/atomic/proceed.html>.

Eissner, W., Jones, M., Nussbaumer, H., 1974. Comput. Phys. Commun. 8, 270.

Fernley, A., Taylor, K.T., Seaton, M.J., 1987. J. Phys. B 20, 6457.

Hillier, J., 2005. Chandra Proposal ID \#07200982, NASA ADS 2005chan.prop.1834H.

Hummer, D.G., Berrington, K.A., Eissner, W., Pradhan, A.K., Saraph, H.E., Tully, J.A., 1993. Astron. Astrophys. 279, 298.

Lee, J.C., Ogle, P.M., Canizares, C.R., Marshall, H.L., Schulz, N.S., Morales, R., Fabian,

A.C., Iwasawa, K., 2001. ApJ 554, L13.

Nahar, S.N., 1996. Phys. Rev. A 53, 2417. 
Nahar, S.N., 2002. Astron. Astrophys. 389, 716.

Nahar, S.N., 2004. Phys. Rev. A 69, 042714-1.

Nahar, S.N., Pradhan, A.K., 1992. Phys. Rev. Lett. 68, 1488.

Nahar, S.N., Pradhan, A.K., 1994. Phys. Rev. A 49, 1816.

Nahar, S.N., Pradhan, A.K., 2000. Phys. Scr. 61, 675.

Nahar, S.N., Pradhan, A.K., 2006. Phys. Rev. A 73, 062718-1.

Ness, J.-U., Brickhouse, N.S., Drake, J.J., Huenemoerder, D.P., 2003. ApJ 598, 1277.

Oelgoetz, J., Pradhan, A.K., 2001. MNRAS 327, L42.

Oelgoetz, J., Pradhan, A.K., 2004. MNRAS 354, 1093.

Peach, G., Saraph, H.E., Seaton, M.J., 1988. J. Phys. B 21, 3669.

Pradhan, A.K., Chen, Guo Xin, Nahar, S.N., Zhang, H.L., 2001. Phys. Rev. Lett. 87, 183201.

Pradhan, A.K., Zhang, H.L., 1997. J. Phys. B 30, L571. Romanik, C., 1988. ApJ 330, 1022.

Sakimoto, K., Terao, M., Berrington, K.A., 1990. Phys. Rev. A 42, 291.

Scott, N.S., Burke, P.G., 1980. J. Phys. B 12, 4299.

Scott, N.S., Taylor, K.T., 1982. Comput. Phys. Commun. 25, 347.

Shull, J.M., van Steenberg, M., 1982. ApJS 48, 95 (values are fitted from the works of Jacobs et al. as referenced in their papers).

Verner, D.A., Ferland, G., 1996. ApJS 103, 467.

Zhang, H.L., Nahar, S.N., Pradhan, A.K., 1999. J. Phys. B 32, 1459. 